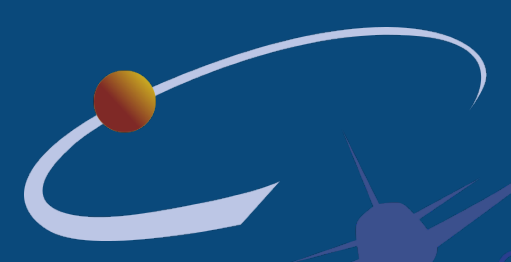

I JPM

\title{
Aerodynamic Optimisation Based on Stability and Sensitivity Analysis
}

\author{
Doctoral Thesis
}

By

Yinzhu Wang

Aerospace Engineer

June 2020 

Departamento de Matemática Aplicada a la Ingeniería Aeroespacial Escuela Técnica Superior de Ingeniería Aeronáutica y del Espacio

\title{
Aerodynamic Optimisation Based on Stability and Sensitivity Analysis
}

\author{
by \\ Yinzhu Wang \\ Aerospace Engineer
}

Advisors:

Eusebio Valero Sánchez

Ph.D. in Aeronautical Engineering

Esteban Ferrer Vaccarezza

Ph.D. in Engineering

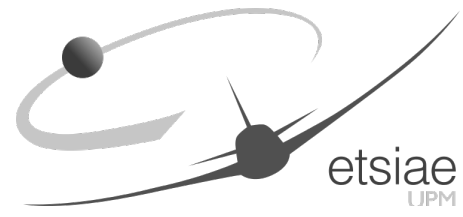

Madrid, June 2020 
This thesis has received funding from the European Union's Horizon 2020 Research and Innovation Program under the Marie Skłodowska-Curie grant agreement No 675008. 


\section{POLITÉCNICA}

El tribunal nombrado por el Sr. Rector Magnífico de la Universidad Politécnica de Madrid, el día de de $20 \ldots . . .$.

Presidente:

Vocal:

Vocal:

Vocal:

Secretario:

Suplente:

Suplente:

Realizado el acto de defensa y lectura de la Tesis el día 25 de octubre de 2019 en la Escuela Técnica Superior de Ingeniería Aeronáutica y del Espacio (ETSIAE-UPM).

Calificación.

EL PRESIDENTE

LOS VOCALES

EL SECRETARIO 

To my loving parents, and Xueqian. 



\title{
Aerodynamic Optimisation Based on Stability and Sensitivity Analysis
}

\begin{abstract}
Aerodynamic optimisation has become a valuable tool the the deign of efficient configurations. However, the usage of aerodynamic optimisation to delay the onset of unsteadiness has remained elusive, even for simple flow.

Flow stability analysis predicts how small flow perturbations grow or decay with respect to an equilibrium flow solution (base flow), providing information about the onset of physical mechanisms responsible for unsteadiness, which can help to determine means by which to control it. The linear stability community has developed tools to analysis the onset of least stable modes, which have been popularised as adjoint-based sensitivity analysis. Sensitivity analysis identifies the regions of the flow that, if properly modified, lead to the greatest damping (or amplification) of the feature responsible for the instability.

In this work, taking advantage of aerodynamic optimisation, stability analysis and sensitivity analysis, an aerodynamic optimisation tool is developed aiming at seeking optimal flow control strategies (both passive and active), based on stability analysis and sensitivity analysis. The main idea of this work is to identify an instability, find the mechanism (mode) that is causing the instability, locate the most sensitive region of the least stable mode, modify the boundary or flow field to manipulate and delay the unsteady onset of this mode. With optimisation methods, it is possible to find a most suitable strategy for the modification to the boundary or the flow field.

To achieve this, stability analysis is used to identify the least stable modes, sensitivity analysis is used to locate the region in the flow field where modifications will most affect these modes. Aided with a variety of techniques, optimisation investigation is conducted on a stability problem. In this thesis, geometric parametrisation is used to represent the geometric with a limited number of parameters, a mesh deformation tool based free form deformation (FFD) is developed, avoiding generating large numbers of similar meshes from scratch, a dynamically updated surrogate model is developed to ensure the accuracy of the surrogate model, while reducing the sampling points in the design of experiments, a mode tracking scheme is developed to avoid manual checking and idenfying modes of interest, which reduces computational cost and alleviates manual labour, making the optimisation investigations more feasible.

In this thesis, the stability analysis and sensitivity analysis are applied to different problems and identified the modes that are causing instabilies, and the structural sensitivity map corresponding to the modes are obtained, which point out the regions where these modes are most affected. The location of the most sensitive region, which provides guidance of where the deformation of the shape should be
\end{abstract}


included, and hence supports and guides the geometric parametrisation for an optimisation investigation. Through optimisation, which is aided with a dynamically updated surrogate model, without excessive computational cost, optimal flow control strategies are obtained. The thesis explores these techniques for a contraction channel, a smooth bump modelling a low pressure turbine.

Keywords: Aerodynamic optimisation, stability analysis, sensitivity analysis, passive/active flow control. 


\section{Aerodynamic Optimisation Based on Stability and Sensitivity Analysis}

\section{Resumen}

La optimización aerodinámica se ha convertido en una herramienta valiosa para el diseño de configuraciones eficientes. Sin embargo, el uso de la optimización aerodinámica para retrasar la aparición de inestabilidad ha sido difícil, incluso para un flujo simple.

El análisis de estabilidad de flujo predice cómo crecen o decaen las pequeñas perturbaciones de flujo con respecto a una solución de flujo de equilibrio (flujo base), proporcionando información sobre el inicio de los mecanismos físicos responsables de la inestabilidad, que pueden ayudar a determinar los medios para controlarla. La comunidad de estabilidad lineal ha desarrollado herramientas para analizar la aparición de modos menos estables, que se han popularizado como análisis de sensibilidad basado en adjuntos. El análisis de sensibilidad identifica las regiones del flujo que, si se modifican adecuadamente, conducen a la mayor amortiguación (o amplificación) de la característica responsable de la inestabilidad.

En este trabajo, aprovechando la optimización aerodinámica, el análisis de estabilidad y el análisis de sensibilidad, se desarrolla una herramienta de optimización aerodinámica con el objetivo de buscar estrategias de control de flujo óptimas (tanto pasivas como activas), basadas en análisis de estabilidad y análisis de sensibilidad. La idea principal de este trabajo es identificar una inestabilidad, encontrar el mecanismo (modo) que está causando la inestabilidad, localizar la región más sensible del modo menos estable, modificar el límite o el campo de flujo para manipular y retrasar el inicio inestable de este modo. Con los métodos de optimización, es posible encontrar la estrategia más adecuada para la modificación del límite o del campo de flujo.

Para lograr esto, el análisis de estabilidad permite identificar los modos menos estables y el análisis de sensibilidad ubica la región en el campo fluido donde las modificaciones afectarán más estos modos. Se utilizan distintas técnicas para completar el ciclo de optimización, por ejemplo, la parametrización geométrica se utiliza para representar la geometría con un número limitado de parámetros. Se desarrolla una herramienta de deformación de malla basada en la deformación de forma libre (FFD), evitando generar grandes cantidades de mallas similares desde cero. Se desarrolla un modelo reducido actualizado dinámicamente para garantizar su precisión y eficiencia en el ciclo de optimización, al tiempo que se reducen los puntos de muestreo en el diseño de experimentos. Finalmente, se desarrolla un esquema de seguimiento de los modos en el proceso de optimización para evitar la verificación manual, lo que reduce el costo computacional haciendo que las investigaciones de optimización sean más factible. 
En esta tesis, el análisis de estabilidad y el análisis de sensibilidad se aplican a diferentes problemas e identifican los modos que causan inestabilidades, y se obtiene el mapa de sensibilidad estructural correspondiente a los modos, que señala las regiones donde estos modos son los más afectados. La ubicación de la región más sensible, que proporciona una guía de dónde debe incluirse la deformación de la forma y, por lo tanto, apoya y guía la parametrización geométrica para una investigación de optimización. Mediante la optimización, que se ayuda con un modelo sustituto actualizado dinámicamente, sin un costo computacional excesivo, se obtienen estrategias de control de flujo óptimas. La tesis explora estas técnicas para un canal de contracción, una protuberancia suave que modela una turbina de baja presión.

Keywords: Optimización aerodinámica, análisis de estabilidad, análisis de sensibilidad, control de flujo pasivo / activo. 


\section{Acknowledgements}

It has not been easy during the last few years working and developing this thesis. Yet, it became much easier with so much support and help both academically and mentally.

First of all, I would like to thank my two thesis advisors, Professor Eusebio Valero and Dr. Esteban Ferrer. They have both been very helpful advisors not only in helping me with my work and life in Madrid. I am very grateful for Eusebio's trust in me when he hired me and gave me this opportunity studying in a very fresh field, for his consideration and help when I just arrived in Madrid and had all sorts of problems, and for his patience when I was stuck on my work and had trouble moving forward. I thank Esteban very much for his constructive academic suggestions, for his devotion in his work, which has a great impact on me, and for pushing me forward on my work when I was distracted to other unimportant weariness.

I would also like to thank Professor Yao Zheng, who was my supervisor in graduate school in Zhejiang University. During my time in Madrid, he helped me with too many things that I cannot count.

I do appreciate Dr. Alejandro Martinez-Cava's help during the doctorate study very much. Sitting next to me in the office must have been a very tough job for him since I constantly had questions to ask. He has always been instructive and patient with all the explanations on the theoritical basics of stability analysis and sensitivity analysis. Without him, it would have been much harder to start most of the work included in this thesis.

I also thank Professor Guillermo Paniagua hosting me in Purdue University. I am very grateful for his help with my settling down at first and with my work later in Purdue University. I thank Dr. Jorge Saavedra very much for explaining his work and help in the paper we drafted together. It was a pleasure with with them.

I have been very lucky to be a part of the Department of Applied Mathematics in Aerospace Engineering. Everyone in this group has been very nice and helpful to me. Tere, Andrés, Binghua, Alex, Jesús, Juan, Diego, Miguel, Marta, Mariola, Carlos, Vicky, Ignacio, Javi, José, Mario, Bartolo, Jorge, Iván and Fer. Thank you all very much for having me as a part of the team and for all the inspirations. I am also very grateful to Patricia Alcanda, Inga Drevile, Carmen Gascó and María Jesús for all the administrative support.

I want to thank all my families for their support. I could always find sanctuary when I was confused or lost. I especially thank my mother and father for not urging me into marriage and for pretending so hard to support me when I chose to start working on the PhD.

To all my friends, thank you so much for being around. I am really lucky to have you. I am very grateful to Jin, Fei, Tao, Mingjun, Liang, for your friendship throughout the years, and for always being there for me. I also thank Andrés, for 
all we shared, for all the experiences we had; Binghua, for all the help and comfort you offered; all the guys in SSeMID, Alex, Andrés, Catherine, Fabio, Francesco, Guillaume, Henrique, Iván, Javier, Julian, Luigi, Petr, Prabhat, Souvik and Thibaut, for all the fun we had, for the working atmosphere you created in this project and for helping me through hard times.

Xueqian, you are the one factor that makes this wonderful world even more so. Thank you for everything! Yinzhu, as dumb as you are, you made it through. Thank you for not giving up even during the hardest times!

In the end, I am very grateful to Universidad Politécnica de Madrid (UPM) for hosting me in the last few years. I would like to thank all the European tax payers and the European Commission for funding this work with Marie Skłodowska-Curie grant No. 675008. 


\section{Contents}

Contents $\quad$ xiii

$\begin{array}{ll}\text { List of Figures } & \text { XV }\end{array}$

List of Tables $\quad$ xix

1 Introduction $\quad 1$

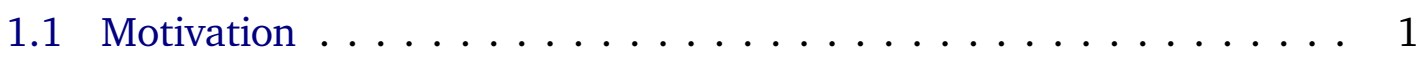

1.2 Research objectives and significance . . . . . . . . . . . 3

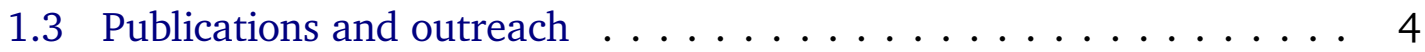

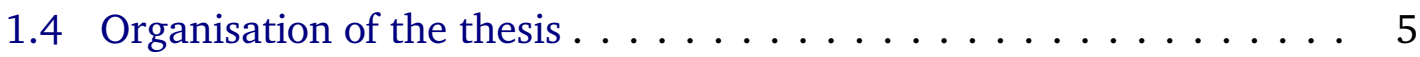

2 Governing Equations and Numerical Solution $\quad 7$

2.1 The Navier-Stokes equations . . . . . . . . . . . . . . . . 8

2.2 Turbulence models . . . . . . . . . . . . . . . . . . . 9

2.3 Spatial discretisation . . . . . . . . . . . . . . . 17

2.4 Temporal discretisation . . . . . . . . . . . . . . . . . . . 20

2.5 Multigrid technique .................... 20

3 Stability Analysis and Sensitivity Analysis 23

3.1 Stability analysis . . . . . . . . . . . . . . . 23

3.2 Adjoint global eigenvalue problem . . . . . . . . . . . . . . 31

3.3 Structural sensitivity analysis . . . . . . . . . . . . 32

4 Aerodynamic Optimisation 35

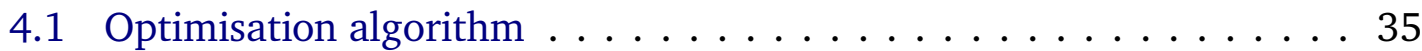

4.2 Surrogate models . . . . . . . . . . . . . . . . . . 37

4.3 Design of experiments . . . . . . . . . . . . . . . . . . . . . . . . . . . . . 49

4.4 Parametric geometry representation . . . . . . . . . . . . . . . . . . 40

4.5 Mesh deformation: freeform deformation . . . . . . . . . . . . . . . 40

4.6 Eigenmode tracking scheme . . . . . . . . . . . . . . . . 41

4.7 Summary of aerodynamic optimisation based on stability analysis and sensitivity analysis . . . . . . . . . . . . . . . 42 
5 Enhanced Stability of Flow through Contraction Channels 45

5.1 Problem description . . . . . . . . . . . . . . . 46

5.2 Base flow and onset of asymmetries . . . . . . . . . . . 48

5.3 Optimisation . . . . . . . . . . . . . . . 57

5.4 Summary and conclusions . . . . . . . . . . . . 72

6 Active flow control optimisation in low-pressure turbines 75

6.1 Problem description . . . . . . . . . . . . . . 76

6.2 Numerical results . . . . . . . . . . . . . . . . . . . . . 78

6.3 Aerodynamic optimisation to find the optimal flow control strategy . 85

6.4 Conclusions . . . . . . . . . . . . . . . . . 97

7 Conclusions and Outlook 99

7.1 Conclusions and Summary . . . . . . . . . . . . . . . . . . . . . . . . . . . . . . . . . .

7.2 Outlook . . . . . . . . . . . . . . . . . . 100

$\begin{array}{ll}\text { Bibliography } & 101\end{array}$ 


\section{List of Figures}

2.1 Control volumes around neighboring points. . . . . . . . . . . . . . 19

4.1 Flow chart of a simple genetic algorithm. . . . . . . . . . . . . 36

4.2 Construction of the surrogate model. . . . . . . . . . . . . . 38

4.3 Three-parameter full factorial designs, with 3 and 4 levels. . . . . . 39

4.4 Examples of three-parameter orthogonal designs, with 3 and 4 levels. 40

4.5 Optimisation flowchart. . . . . . . . . . . . . . . . . 43

5.1 Geometry and the characteristic lengths. . . . . . . . . . . . . . 47

5.2 Bifurcation diagram for contraction ratios $C=2,4$ and 8, illustrated using the reattachment lengths $L_{3}$ of downstream tip corner eddy for varying Reynolds numbers. . . . . . . . . . . . . . . . . 51

5.3 Horizontal velocity component $U$ (a,b) and streamlines (c,d) for $R e=1000$ and $R e=2000$, showing symmetric and asymmetric recirculation bubbles, respectively. Contraction ratio is $C=4$ in all

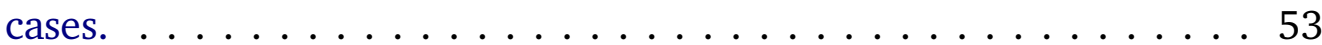

5.4 Recirculation bubbles for contraction ratio $C=4$ at $R e=800$ (dash dot dot), $R e=1200$ (dash dot), $R e=1600$ (solid) and $R e=2000$

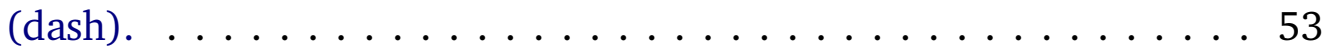

5.5 Growth rates (real part of eigenvalues) against Reynolds numbers,

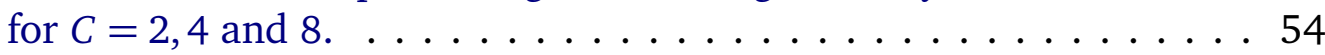

5.6 Eigenvalue spectra for $R e=1000$ and 2000 for contraction ratio $C=$ 4. The least stable mode is marked with a filled square. . . . . . . . . 55

5.7 Direct (a,b) and adjoint (c,d) eigenmodes for $R e=1000$ and $R e=$ 2000, for contraction ratio $C=4$. All show horizontal velocity component $u \ldots \ldots \ldots \ldots \ldots$. . . . . . . . . . . . . . 56

5.8 Structural sensitivity map, Production of kinetic energy and lift-up kinetic energy perturbation, at $R e=2000$ for contraction ratio $C=4$. Red lines indicate the edge of recirculation regions. . . . . . . . . . 57

5.9 Super-elliptic curves with different degrees, with parameters $(a, b, 1 / p) .59$ 
5.10 Geometry parametrisation using the parameters $\boldsymbol{p}=\left(p_{1}, p_{2}, p_{3}\right)$ at the contraction stage. . . . . . . . . . . . . . . . 60

5.11 Examples of geometries using the geometry parametrisation $\boldsymbol{p}=$

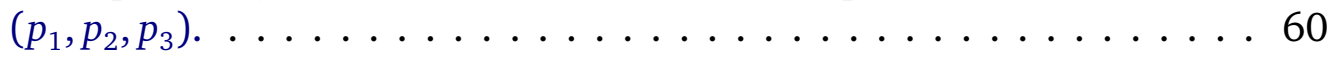

5.12 Examples of mesh deformation for the geometries depicted in figure

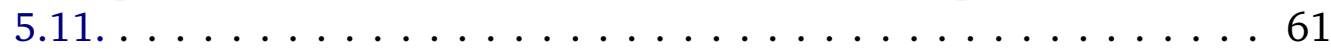

5.13 Convergence process of the optimisation. . . . . . . . . . . . . 62

5.14 Original and optimised geometries for contraction ratio $C=2$ : eigenvalue spectra, eigenmodes, base flows, structural sensitivities and lift-up perturbation kinetic energy terms. Red lines indicate the edge of recirculation regions. . . . . . . . . . . . . . . . . . . 64

5.15 Original and optimised geometries for contraction ratio $C=4$ : eigenvalue spectra, eigenmodes, base flows, structural sensitivities and lift-up perturbation kinetic energy terms. Red lines indicate the edge of recirculation regions. . . . . . . . . . . . . . 65

5.16 Original and optimised geometries for contraction ratio $C=8$ : eigenvalue spectra, eigenmodes, base flows, structural sensitivities and lift-up perturbation kinetic energy terms. Red lines indicate the edge of recirculation regions. . . . . . . . . . . . . . . 66

5.17 Parameter-sensitivity of the growth rate to variations in the geometric parameters $\boldsymbol{p}=\left(p_{1}, p_{2}, p_{3}\right)$, near the optimised configuration. . . . 68

5.18 Bifurcation diagram of the optimised geometries for contraction ratios $C=2$ and 4, illustrated using the reattachment lengths $L_{3}$ of downstream tip corner eddy for varying Reynolds numbers. . . . . . . 69

5.19 Flow field in the optimised geometry, $C=8$. . . . . . . . . . . . . 69

5.20 The relation between drag coefficient and Reynolds number. . . . . . 70

5.21 Verification of the results with three-dimensional domain. . . . . . . 73

6.1 Geometric configuration of the channel with a wall-mounted hump. . 78

6.2 Mesh. . . . . . . . . . . . . . . . . . . . . . . 79

6.3 Detachment and reattachment locations against inlet total pressure . 80

$6.4 u_{x}$ with different inlet total pressure. . . . . . . . . . . 81

6.5 Eigenvalue spectrum, $P_{0}=100500 \ldots \ldots \ldots \ldots \ldots$. . . . . 82

6.6 Eigenmode and its sensitivity map corresponds to $\sigma=(4.272,0) \ldots .83$

6.7 Eigenmode and its sensitivity map corresponds to $\sigma=(-0.5436,2.366) .84$

6.8 Geometric configuration used in the optimisation (zoomed in near the hump), 2-parameter. . . . . . . . . . . . . . . 86

$6.9 u_{x}$, optimal design, 2-parameter, objective: pressure loss. . . . . . . . 87

6.10 The eigenvalue spectrum of the optimal configuration, 2-parameter, objective: pressure loss. . . . . . . . . . . . . . . . . 88

6.11 The most unstable eigenmode of the optimal design, 2-parameter, objective: pressure loss. . . . . . . . . . . . . . . . . . . . . . 89 
6.12 The structural sensitivity map of the most unstable mode, 2-parameter, objective: pressure loss. . . . . . . . . . . . . . . . . . . . . . . . 89

6.13 The eigenvalue spectrum of the optimal configuration, 2-parameter, objective: amplification rate. . . . . . . . . . . . . . . 90

6.14 The most unstable eigenmode of the optimal design, 2-parameter, objective: amplification rate. . . . . . . . . . . . . . . 91

6.15 The structural sensitivity map of the most unstable mode, 2-parameter, objective: amplification rate. . . . . . . . . . . . . . . 91

$6.16 u_{x}$, optimal design, 2-parameter, objective: amplification rate. . . . 92

6.17 Geometric configuration used in the optimisation (zoomed in near the hump), 3-parameter. . . . . . . . . . . . . . . . . . . 92

$6.18 u_{x}$, optimal design, 3-parameter, objective: pressure loss. . . . . . . 93

6.19 The eigenvalue spectrum of the optimal configuration, 3-parameter, objective: pressure loss. . . . . . . . . . . . . . . . . . . . 94

6.20 The most unstable eigenmode of the optimal design, 3-parameter, objective: pressure loss. . . . . . . . . . . . . . . . . . . 94

6.21 The structural sensitivity map of the most unstable mode, 3-parameter, objective: pressure loss. . . . . . . . . . . . . . . . . . 95

6.22 The eigenvalue spectrum of the optimal configuration, 3-parameter, objective: amplification rate. . . . . . . . . . . . . . . 95

6.23 The most unstable eigenmode of the optimal design, 3-parameter, objective: amplification rate. . . . . . . . . . . . . . . 96

6.24 The structural sensitivity map of the most unstable mode, 3-parameter, objective: amplification rate. . . . . . . . . . . . . . 96

$6.25 u_{x}$, optimal design, 3-parameter, objective: amplification rate. . . . . 97 



\section{List of Tables}

4.1 Parameters selected for the Genetic Algorithm. . . . . . . . . . 37

5.1 Geometry parameters and separation/reattachment lengths. . . . . 48

5.2 Grid convergence: Separation and reattachment lengths $L_{1}, L_{2}$ and $L_{3}$ for contraction ratio $C=2 \ldots \ldots \ldots \ldots \ldots \ldots$

5.3 Detailed parameters of the locally refined mesh. . . . . . . . . . 49

5.4 Grid convergence fro eigenvalues: real part $\left(\sigma_{R}\right)$ of the most unstable asymmetric mode, for contraction ratio $C=2 \ldots \ldots \ldots \ldots$. . . 49

5.5 Krylov subspace dimensions convergence for eigenvalues: Real part $\left(\sigma_{R}\right)$ of the most unstable asymmetric mode, for contraction ratio

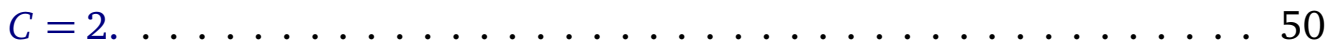

5.6 Parameters selected for the Genetic Algorithm. . . . . . . . . . . 58

5.7 Range for the geometry parameters (or design space): $\boldsymbol{p}=\left(p_{1}, p_{2}, p_{3}\right) .59$

5.8 Total number of iterations and computational time taken for the op-

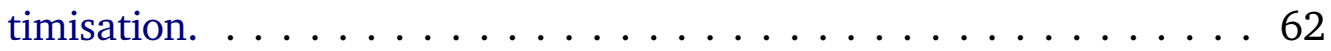

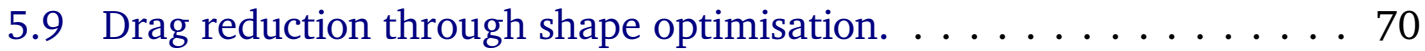

$5.10 L_{3}$ against spanwise size, $R e=1600, C=4 \ldots \ldots \ldots \ldots \ldots 71$

$5.11 L_{3}$ against spanwise size, $R e=1600, C=4 \ldots \ldots \ldots \ldots \ldots 72$

6.1 Parameters describing the geometry and charactorising the flow. . . 78

6.2 Boundary conditions. . . . . . . . . . . . . . . . 79

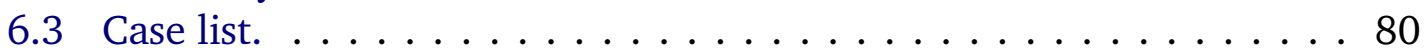

6.4 Design of experiment. . . . . . . . . . . . . . . . 86

6.5 Design of experiment. . . . . . . . . . . . . . . . 92

6.6 Summary of the different optimisation investigations. . . . . . . 98 



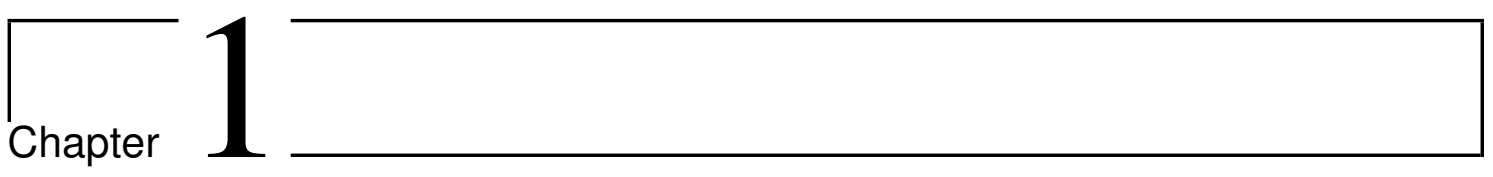

\section{Introduction}

\section{Contents}

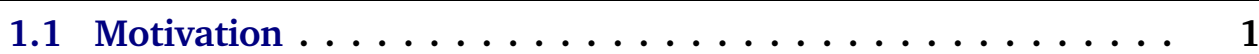

1.1.1 Aerodynamic optimisation $\ldots \ldots \ldots \ldots 1$

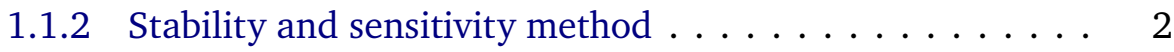

1.2 Research objectives and significance . . . . . . . . . 3

1.3 Publications and outreach . . . . . . . . . . 4

1.4 Organisation of the thesis $\ldots \ldots \ldots \ldots \ldots \ldots$

\subsection{Motivation}

In the early days of aeronautical industry, limited by methodologies and analytical tools, aerodynamic designs is dependent of designers' experience and the "cut and try" skeme. The designs were modified and tested over and over again. This process consumed a great deal of money, time and efforts, but yet did not produce an overall optimal or even feasible design.

\subsubsection{Aerodynamic optimisation}

With the development of computational fluid dynamics (CFD) and high performance computers, numerical simulations have replaced most of the wind tunnel tests, which makes the design process much shorter and leads to much more dedicated design. Based on numerical simulation technology, reverse design [42, 65, 100] and aerodynamic optimisation $[4,51,58]$ methods are developed. The reverse design method, based on a given velocity/pressure profile on the target surface, iteratively modifies the aerodynamic shape to close in to the desired velocity/pressure profile, to get the aerodynamic shape that meets the aerodynamic requirement. With the velocity/pressure profile properly selected, this method could lead to very good result efficiently. Since the introduction of the reverse design 
method by Tranen [107] in transonic airfoil design, it has shown a lot of its applications in the industry $[30,75,114]$. However, since a proper velocity/pressure profile is essential to this method, and the limitation of this method in dealing with aerodynamic and geometrical contraints, the application of this reverse design has been greatly limited. Meanwhile, the industry has put a lot of efforts in reducing the expenses and time cost, which has led to the boost of the development of aerodynamic optimisation methods.

Aerodynamic optimisation replaces an aerodynamic design problem with an optimisation problem, and replaces a design objective with an optimisation objective. An aerodynamic shape with optimal aerodynamic performance is obtained by optimisation algorithms. To conduct aerodynamic optimisation, a proper geometry parametrisation method, a mesh deformation/regeneration and a numerical tool for aerodynamic performance analysis need to be introduced. In comparison with the reverse design method, the aerodynamic optimisation method obtains aerodynamic shapes, which meet the aerodynamic requirements, with less influence by personals. Even with a proper given velocity/pressure profile, the aerodynamic optimisation method is capable of taking the given velocity/pressure profile as an objective in an optimisation process, thus taking the reverse design problem as an optimisation problem.

In the last decades, aerodynamic shape optimisation has become a valuable tool for the design of efficient lifting surfaces and even entire aircraft [79].

The advances in computing power and development of more accurate computational fluid dynamics solvers have promoted optimal shape design [52]. In most recent research, Pralits and Hanifi[83], Flinois and Colonius[27] and Xiao and Papadakis[115], Martínez-Cava[70] have successfully applied aerodynamic optimisation to optimal flow control stragies. However, the use of shape optimisation to delay the onset of unsteadiness has remained elusive, even for simple flows. One of the reasons is that every function evaluation requires two steps, which are both considerably costy when it comes to computation. First, a steady base flow (steady Navier-Stokes base flow simulation) needs to be obtained and, second, an eigenvalue system needs to be solved to find the stability of perturbations. The combination of these two steps leads to function evaluations that can become prohibitive.

\subsubsection{Stability and sensitivity method}

Flow stability analysis [95] predicts how small flow perturbations grow or decay with respect to an equilibrium flow solution (the base flow), providing information about the onset of asymmetries or unsteadiness, the physical mechanism associated to the perturbation responsible for the bifurcation, and can help to determine means by which to control it. The application of stability analysis of fluid dynamics problems is extensive and includes, but not limited to: sudden expansions $[23,24,37,97]$, sudden contractions $[14,15]$, geometries with both an expansion 
and a contraction [73], X-junctions [64], or more recently T-junctions [12, 61, 106]. The physical mechanism behind the onset of asymmetry, in these cases, is a stationary unstable global mode, flows behind bumps [29, 78], behind backward facing steps [6, 63, 69], in boundary layer recirculation bubbles [87] and in lid-driven cavities $[1,39]$.

One of the main results obtained through the stability analysis is the determination of flow bifurcation, either to new steady or unsteady configuration. In this regards, the linear stability community has developed tools to control the onset of bifurcations, which have been popularised as adjoint-based sensitivity analysis. Sensitivity analysis identifies the regions of the flow that, if modified, lead to the greatest damping (or amplification) of the feature responsible for the instability. The numerical study of flow sensitivity relies on the use of adjoint solutions [66]. The importance of adjoint and sensitivity maps, together with the mathematical tools required for the study of the sensitive flow regions, to different parameters, were first introduced by Hill[45], and have been used extensively to examine the receptivity to internal and external modifications [32, 66, 69], and also by the authors Ferrer et al. [25, 26], Browne et al.[10], Iorio et al.[56], Gonzalez et al.[35]. Having determined the most sensitive flow regions, it becomes relatively simple to apply a flow control technique. Passive control introduces inactive objects (e.g. a small cylinder), which modifies the flow to stabilise or modify the frequency of particular flow features [69]. Useful active flow control may also be derived from sensitivity maps to control undesirable instabilities [98].

Finally, it is important to highlight that, sensitivity analysis relies on linear modal flow structures and hence are strictly valid under linear assumptions (e.g. small variations from equilibrium). For example, if one applies sensitivity to geometrical modifications, it is necessary to assume that these modification do not alter significantly the base flow, used to obtain the modal structures. For large modifications, the base flow changes and the sensitivities may not effectively guide the flow control design. To overcome this limitation, an alternative to sensitivity analyses is to use a full optimisation cycle, where the modal eigenvalues constitute the objective function, and the associated base flow is recomputed at each function evaluation.

In what follows, we consider the growth rates of the modes of interest as the target function and attempt to damp these through geometric variations and active flow control strategies (i.e. shape optimisation). The results are analysed and assessed using linear stability analysis and sensitivity analysis.

\subsection{Research objectives and significance}

The main objectives of this thesis are to obtain sensitivity maps of unstable modes under the effect of design variables or external influence, such as surface/shape deformation and injections or suctions, and to create an optimisation tool for aero- 
dynamic optimisation problems aiming at optimal flow control based on stability analysis and sensitivity analysis. This work contributes to provide an efficient optimisation tool that finds optimal passive or active flow control strategies, by seeking the minimal value of the amplification rate of an unfavourable mode. When the amplification rate is below zero, the mode decays in the domain through time and this mode is made stable. In aerodynamic optimisation investigations, the computational cost is very considerable. A dynamically updated surrogate model is utilised to reduce the computational cost, while keeping the accuracy of the surrogate models. The computational time is greatly reduced in the optimisation procedure. Using structural sensitivity analysis, the sensitivity of shape deformation to the most unstable mode is obtained. Based on the sensitivity map and stability analysis, the optimal shape of the contraction channels are obtained, making the flow stable for a much larger range of Reynolds numbers.

Based on stability analysis, aerodynamic optimisation investigations have been conducted for optimal active flow control strategy in low-pressure turbines, giving optimal injection location, angle and blow rate over the turbine and minimising the pressure loss in the flow passage.

\subsection{Publications and outreach}

During the work of this thesis, various publications and presentations have be made. Publications:

- Y. Wang, J. Saavedra, E. Esteban, G. Paniagua and E. Valero, "Optimisation in Active Flow Control in Low-Pressure Turbines", submitted for publication.

- Y. Wang, E. Ferrer, A. Martínez-Cava, Y. Zheng and E. Valero, "Enhanced stability of flows through contraction channels: combining shape optimisation and linear stability analysis", Physics of Fluids, 31, 2019.

- A. Martínez-Cava, Y. Wang, J. de Vicente and E. Valero, "Pressure bifurcation phenomenon on supersonic blowing trailing edge", AIAA Journal, 57(1), 2018.

Presentations and preceedings:

- Y. Wang and A. Martínez-Cava, "Eigenmode optimisation based on stability analysis and sensitivity analysis", EUROGEN 2017, Madrid, September 2017.

- Y. Wang, A. Martínez-Cava, E. Valero, Y. Zheng and E. Ferrer, "Eigenmode optimisation of a contraction channel based on stability analysis", ECCM-ECFD 2018, Glasgow, June 2018.

- Y. Wang, J. Saavedra, E. Ferrer, G. Paniagua and E. Valero, "Optimisation in active flow separation control in low-pressure turbines", 8th EUCASS, Madrid, July 2019. 


\subsection{Organisation of the thesis}

This rest of the thesis is organised as:

Chapter 2 summarises the basic concepts of computational fluid dynamics. In the first part, the Navier-Stokes equations are dicussed. In the second part, turbulence models used in this thesis are discussed, followed by spatial and temporal discretisation and the multi-grid techneque that speeds up the convergence.

Chapter 3 introduces the basics of stability analysis and sensitivity analysis. First, stability analysis is discussed and then adjoint global eigenvalue problem is introduced, followed by the structural sensitivity analysis.

Chapter 4 concludes the essential techniques used in aerodynamic optimisation. First, an introduction of the optimisation algorithm, GA, is given. Then surrogate modelling and a dynamically updated surrogate model is introduced. Followed by the geometry parametrisation and mesh deformation for shape optimisation is discussed. For optimisation investigations based on stability analysis, an eigenmode tracking scheme is introduce. Finally, a summary of aerodynamic optimisation based on stability analysis and sensitivity analysis is given.

Chapter 5 gives an example of aerodynamic shape optimisation on a contraction channel to supress the asymmetry in the channel based on stability analysis and sensitivity analysis. In the first part, a despription of the problem is given. Then the base flow and onset of asymmetries are discussed. Followed by the discussion of the results of the optimisation. Finally, The summary and conclusions of the aerodynamic investigation on this problem is given.

Chapter 6 gives another example of aerodynamic optimisation on active flow control on a low-pressure turbine based on stability analysis and sensitivity analysis.

Finally, the summary of the thesis and outlook about the work described in the thesis are given in Chapter 7. 

$\Gamma_{\text {Chapter }}$

\section{Governing Equations and Numerical Solution}

\section{Contents}

2.1 The Navier-Stokes equations $\ldots \ldots \ldots \ldots \ldots \ldots$

2.2 Turbulence models $\ldots \ldots \ldots \ldots \ldots \ldots$

2.2.1 Spalart-Allmaras turbulence model . . . . . . . . . . . 10

$2.2 .2 k-\omega \operatorname{model} \ldots \ldots \ldots \ldots \ldots \ldots \ldots \ldots$

2.2.3 $\gamma-R e_{\theta t}$ transition transport model . . . . . . . . 13

2.3 Spatial discretisation $\ldots \ldots \ldots \ldots \ldots \ldots \ldots \ldots$

2.3.1 Upwind scheme . . . . . . . . . . . . 17

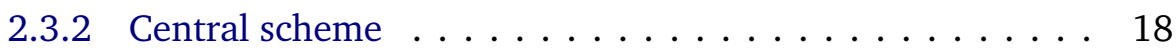

2.4 Temporal discretisation $\ldots \ldots \ldots \ldots \ldots \ldots$

2.5 Multigrid technique . . . . . . . . . . . . . . 20

In aerodynamic optimisation, aerodynamic characteristics related parameters, cost function, constraints, like the lift coefficient and the drag coefficient etc. need to be determined according to specific desing parameters. An optimisation is carried on based on this information to search for an optimal design. Hence, the precision and efficiency of obtaining the aerodynamic characteristics foundamentally affect the efficiency of the optimisation. In this theis, CFD (computational fluid dynamics) is used to obtain the discretised solution as the cost function of an aerodynamic optimisation. The DLR-TAU code is adopted for the simulations, and ansys ICEM is used as the mesh generator for all the work done within the frame of this thesis.

In this section, the governing equations of the flow and the numerical method of solution will be introduced. 


\subsection{The Navier-Stokes equations}

The Navier-Stokes equations for the three dimensional case can be written in conservative form as

$$
\frac{\partial}{\partial t} \iiint_{V} \vec{W} d V=-\iint_{\partial V} \overline{\bar{F}} \cdot \vec{n} d S
$$

where

$$
\vec{W}=\left[\begin{array}{c}
\rho \\
\rho u \\
\rho v \\
\rho w \\
\rho E
\end{array}\right]
$$

is the vector of the conserved quantities. $V$ denotes an arbitrary control volume with the boundary $\partial V$ and the outer normal $\vec{n}$. The flux density tensor $\overline{\bar{F}}$ is composed of the flux vectors in the three coordinate directions:

$$
\overline{\bar{F}}=\left(\vec{F}_{i}^{c}+\vec{F}_{v}^{c}\right) \cdot \vec{e}_{x}+\left(\vec{G}_{i}^{c}+\vec{G}_{v}^{c}\right) \cdot \vec{e}_{y}+\left(\vec{H}_{i}^{c}+\vec{H}_{v}^{c}\right) \cdot \vec{e}_{z}
$$

in which $e_{x}, e_{y}$ and $e_{z}$ are unit vectors in the coordinate directions. The indices $i$ and $v$ denote the inviscid and the viscous contributions, respectively. The viscous contributions are neglected when considering the Euler equations. the viscous and the inviscid fluxes are

$$
\begin{aligned}
& \vec{F}_{i}^{c}=\left[\begin{array}{l}
\rho u \\
\rho u^{2}+p \\
\rho u v \\
\rho u w \\
\rho H u
\end{array}\right], \quad c_{v}^{c}=\left[\begin{array}{l}
0 \\
\tau_{x x} \\
\tau_{x y} \\
\tau_{x z} \\
u \tau_{x x}+v \tau_{x y}+w \tau_{x z}+\kappa_{l} \frac{\partial T}{\partial x}
\end{array}\right], \\
& \vec{G}_{i}^{c}=\left[\begin{array}{l}
\rho v \\
\rho u v \\
\rho v^{2}+p \\
\rho v w \\
\rho H v
\end{array}\right], \quad \vec{G}_{v}^{c}=\left[\begin{array}{l}
0 \\
\tau_{x y} \\
\tau_{y y} \\
\tau_{y z} \\
u \tau_{x y}+v \tau_{y y}+w \tau_{y z}+\kappa_{l} \frac{\partial T}{\partial y}
\end{array}\right], \\
& \vec{H}_{i}^{c}=\left[\begin{array}{l}
\rho w \\
\rho u w \\
\rho v w \\
\rho w^{2}+p \\
\rho H w
\end{array}\right], \quad \vec{H}_{v}^{c}=\left[\begin{array}{l}
0 \\
\tau_{x z} \\
\tau_{y z} \\
\tau_{z z} \\
u \tau_{x z}+v \tau_{y z}+w \tau_{z z}+\kappa_{l} \frac{\partial T}{\partial z}
\end{array}\right],
\end{aligned}
$$

The pressure is calculated by the equation of state

$$
p=(\gamma-1) \rho\left(E-\frac{u^{2}+v^{2}+w^{2}}{2}\right) .
$$


From equation 2.1, and assuming $\vec{W}$ as constant over each volume, the temporal change of the conservative variables $\vec{W}$ can be written as

$$
\frac{\partial}{\partial t} \vec{W}=-\frac{\iint_{\partial V} \overline{\bar{F}} \cdot \vec{n} d S}{\iiint_{V} d V}
$$

The change of the flow conditions in a control volume $\mathrm{V}$ is given by the flux over the control volume boundary $\partial V$ related to the size of $V$. For a control volume fixed in time and space, equation 2.8 can be written as

$$
\frac{d}{d t} \vec{W}=-\frac{1}{V} \cdot \vec{Q}^{F}
$$

in which $\vec{Q}^{F}$ represents the fluxes over the boundaries of the control volume. if the boundary is divided into $n$ faces, $\vec{Q}^{F}$ is given by

$$
\vec{Q}^{F}=\sum_{i=1}^{n} \vec{Q}_{i}^{F}=\sum_{i=1}^{n}\left(\vec{Q}_{i}^{F, c}-\vec{D}_{i}\right)
$$

in which $\vec{Q}_{i}^{F, c}$ denote the inviscid fluxes over the respective face. hence, in order to determine the temporal change of the flow quantities in a control volume, the convective fluxes over the control volume boundaries have to be determined. For upwind schemes the dissipative terms $\vec{D}_{j}$ are zero, but for central schemes additional dissipative terms have to be computed.

\subsection{Turbulence models}

Due to the extensive scale of CFD problems, which could be extremely large or small, directly solving the Navier-Stokes equations (DNS) could be very computationally expensive. The resolution of the mesh and time steps needed for the problem greatly affect the computational cost. From RANS (Reynolds averaged Navier-Stokes equations) to DNS, the computational cost increases as the number of degrees of freedom required to solve the flow increases. Considering computational accuracy and efficiency, in this thesis, all the simulations are carried out using RANS-based turbulence models.

In this section, the turbulence models utilised in this thesis are given.

\subsubsection{Spalart-Allmaras turbulence model}

The Spalart-Allmaras turbulence model (SA model) has been origiinally presented by Spalart and Allmaras[101] and then revised and developed through the years[3]. 
All SA models adopt a single transport equation for the SA viscosity, $\tilde{v}$, which is related to the kinematic eddy viscosity $v_{t}$ given by

$$
v_{t}=\tilde{v} f_{v 1}
$$

in which

$$
f_{v 1}=\frac{\chi^{3}}{\chi^{3}+c_{v 1}^{3}}
$$

is a damping function, which depends on the turbulence Reynolds number given by the viscosity ratio

$$
\chi=\frac{\tilde{v}}{\bar{v}}
$$

in which $\bar{v}$ is the average kinematic viscosity of the fluid, and $c_{v 1}=7.1$.

In case of rough walls the definition of $\chi$ is modified according to Aupoix and Spalart[5]:

$$
\chi=\frac{\tilde{v}}{\bar{v}}+c_{R 1} \frac{k_{s}}{d},
$$

in which $c_{R 1}=0.5, k_{s}$ is the sand roughness and $d$ is the wall distance.

The transport equation for the SA viscosity is given by

$$
\frac{\partial(\bar{\rho} \tilde{v})}{\partial t}+\frac{\partial}{\partial x_{k}} \bar{\rho} \tilde{v} \tilde{U}_{k}=\bar{\rho} P^{(\tilde{v})}-\bar{\rho} D^{(\tilde{v})}-\bar{\rho} \Phi^{(\tilde{v})}+\bar{\rho} C^{(\tilde{v})}+\bar{\rho} T^{(\tilde{v})}
$$

in which $\bar{\rho} P^{(\tilde{v})}$ represents production, $\bar{\rho} \Phi^{(\tilde{v})}$ represents destruction and $\bar{\rho} D^{(\tilde{v})}$ represents diffusion. $\bar{\rho} C^{(\tilde{v})}$ and $\bar{\rho} T^{(\tilde{v})}$ are modifications for compressibility and turbulence onset, respectively, which are treated more or less optionally. Production, destruction and diffusion are given by

$$
\begin{gathered}
\bar{\rho} P^{(\tilde{v})}=c_{b 1}\left(1-f_{t 2}\right) \tilde{S} \bar{\rho} \tilde{v} \\
\bar{\rho} \Phi^{(\tilde{v})}=\left(c_{\omega 1} f_{\omega}-\frac{c_{b 1}}{\kappa^{2}} f_{t 2}\right) \bar{\rho}\left(\frac{\tilde{v}}{d}\right)^{2} \\
\bar{\rho} D^{(\tilde{v})}=\frac{1}{\sigma}\left\{\frac{\partial}{\partial x_{k}}\left[\bar{\rho}(\bar{v}+\tilde{v}) \frac{\partial \tilde{v}}{\partial x_{k}}\right]+c_{b 2} \bar{\rho} \frac{\partial \tilde{v}}{\partial x_{k}} \frac{\partial \tilde{v}}{\partial x_{k}},\right\}
\end{gathered}
$$

in which, the model coefficients take the following values:

$$
c_{b 1}=0.1355, \quad c_{b 2}=0.622, \quad \sigma=\frac{2}{3}, \quad \kappa=0.41, \quad c_{\omega 1}=\frac{c_{b 1}}{\kappa^{2}}+\frac{1+c_{b 2}}{\sigma} .
$$

In the production term, $\tilde{S}$ is a scalar measure of the velocity gradient that is defined differently for different variants of the SA model. In the destruction tuerm,

$$
f_{\omega}=g\left[\frac{1+c_{\omega 3}^{6}}{g^{6} c_{\omega 3}^{6}}\right]^{1 / 6},
$$


with

$$
g=r+c_{\omega 2}\left(r^{6}-r\right)
$$

controls the decay of $\tilde{v}$ towards the boundary layer edge. The used coefficient values are

$$
c_{\omega 2}=0.3, \quad c_{\omega 3}=2 .
$$

Note that the argument $r$ is defined differently for different model versions.

Finally,

$$
f_{t 2}=c_{t 3} \exp \left\{-c_{t 4} \chi^{2}\right\}
$$

appearing in both producion and dissipation terms, has been designed, in order to suppress turbulence in laminar regions. The values of the related coefficients are

$$
c_{t 3}=1.2, \quad c_{t 4}=0.5
$$

In case the value of $\tilde{v}$ is too low in the free steam, the term, $f_{t 2}$ may lead to an unintended pseudo-laminar behaviour of the model in fully turbulent simulations. It is therefore de-activated in some of the model versions $\left(c_{t 3}=0\right)$. In case of active $f_{t 2}$-term, the far field viscosity ratio must be of the order $\left(\mu_{t} / \bar{\mu}\right)_{\infty}=0.2 \quad \ldots \quad$ 1.3.

The compressibility term

$$
\bar{\rho} C^{(\tilde{v})}=-\frac{1}{\sigma}(\bar{v}+\tilde{v}) \frac{\partial \bar{\rho}}{\partial x_{k}} \frac{\partial \tilde{v}}{\partial x_{k}}
$$

with

$$
g_{t}=\operatorname{MIN}\left(0.1, \partial \Delta u \omega_{t} \Delta x\right),
$$

and the coefficient values

$$
c_{t 1}=1, \quad c_{t 2}=2
$$

shall enhance turbulence onset at a specified trip point. In these equations $d_{t}$ is the distance from the trip point, $\omega_{t}$ is the vorticity at the trip point, $\Delta u$ is the velocity difference relative to the trip point, and $\Delta x$ is the streamwise grid spaceing at the trip[3].

\subsection{2 $k-\omega$ model}

In this context, the Menter SST (shear stress transport) version of $k-\omega$ model is adopted. The Menter SST model is a further improvement of the MBL (enter baseline) model[71]. the Menter baseline model is a blending of the Wilcox $k-\omega$ model in the inner part of the boundary layer and the $k-\varepsilon$ model in the outer part of the boundary layer. The aim is to obtain both the accuracy in the near-wall region of the Wilcox $k-\omega$ model and the freestream independence in the outer part of the boundary layer of the $k-\varepsilon$ model. For this purpose, the $k-\varepsilon$ model is transformed into a $k-\omega$ formulation. The difference between both formulations 
is that an additional cross-diffusion term appears in the $\omega$ equation and that the modelling coefficients are different.

In the baseline model $k$ and $\omega$ are the solution of

$$
\begin{aligned}
\frac{\partial(\rho k)}{\partial t}+\vec{\nabla} \cdot(\rho \vec{u} k)-\vec{\nabla} \cdot\left(\left(\mu+\mu_{t} \sigma_{k}\right) \vec{\nabla} k\right)=\tilde{\mathscr{P}}-\beta_{k} \rho k \omega, \\
\quad \frac{\partial(\rho \omega)}{\partial t}+\vec{\nabla} \cdot(\rho \vec{u} \omega)-\vec{\nabla} \cdot\left(\left(\mu+\mu_{t} \sigma_{\omega}\right) \vec{\nabla} \omega\right) \\
=\frac{\gamma \rho}{\mu_{t}} \mathscr{P}-\beta \rho \omega^{2}+2 \sigma_{\omega 2} \rho \omega^{2}+2 \sigma_{\omega 2}\left(1-F_{1}\right) \frac{\rho}{\omega} \vec{\nabla} k \cdot \vec{\nabla} \omega .
\end{aligned}
$$

The production term in the $k$ equation is limited:

$$
\tilde{\mathscr{P}}=\operatorname{MIN}\left(\mathscr{P}, C_{\text {limit } P_{k}} \varepsilon\right), \quad \varepsilon=\beta_{k} \rho k \omega,
$$

in which $C_{\text {limit }}$ is a user-chosen constant. The production limiter was devised by Menter for in regions of small values for $\omega$, erroneous spikes of $v_{t}$ can occur. There might stem from the term $v_{t} \omega^{-1} \mathscr{S}^{2}$ in the $v_{t}=k / \omega$ equation. This production term can be amplified by small values for $\omega$. As a remedy the production term is limited.

The coefficients $\phi \in\left\{\sigma_{k}, \sigma_{\omega}, \gamma, \beta_{\omega}\right\}$ of the model are interpolated using the blending formula

$$
\phi=F_{1} \phi_{1}+\left(1+F_{1}\right) \phi .
$$

$\phi_{1} \in\left\{\sigma_{k 1}, \sigma_{\omega 1}, \gamma_{1}, \beta_{\omega 1}\right\}$ as in the inner layer, particularly for Menter SST model

$$
\sigma_{k 1}=0.85, \quad \sigma_{\omega 1}=0.5, \quad \gamma_{1}=0.555556, \quad \beta_{\omega 1}=\beta_{k}\left(\gamma_{1}+\frac{\sigma_{\omega 1} \kappa^{2}}{\sqrt{\beta_{k}}}\right)
$$

$\phi_{2} \in\left\{\sigma_{k 2}, \sigma_{\omega 2}, \gamma_{2}, \beta_{\omega 2}\right\}$ as in the outer layer are given by

$$
\sigma_{k 2}=1.0, \quad \sigma_{\omega 2}=0.857, \quad \gamma_{2}=0.44, \quad \beta_{\omega 2}=\beta_{k}\left(\gamma_{2}+\frac{\sigma_{\omega 2} \kappa^{2}}{\sqrt{\beta_{k}}}\right),
$$

in which $\kappa=0.41, \beta_{k}=0.09$ and using the blending function $F_{1}$ given by

$$
\begin{aligned}
F_{1}=\tanh \left(\arg _{1}^{4}\right), \quad \arg g_{1} & =\operatorname{MIN}\left(\operatorname{MAX}\left(\frac{\sqrt{k}}{\beta_{k} \omega y}, \frac{500 v}{y^{2} \omega}\right), \frac{4 \rho \sigma_{\omega 2} k}{C D_{k \omega} y^{2}}\right), \\
C D_{k \omega} & =\operatorname{MAX}\left(2 \rho \sigma_{\omega 2} \frac{1}{\omega} \vec{\nabla} k \cdot \vec{\nabla} \omega, 10^{-20}\right) .
\end{aligned}
$$

in which substitution gives $\beta_{\omega 1}=0.07522$ and $\beta_{\omega 2}=0.08282$.

The function $F_{1}$ controls the blending of the model coefficients and the cross diffusion term. Therefore, $F_{1}$ has to be one in the near-wall region and in the logarithmic layer and has to tapper off to zero well within the wake region of the boundary layer in order to prevent the freestream dependence of the $k-\omega$ model. 
Specially for Menter SST model, shear-stress correction is introduced as

$$
\mu_{t}=\operatorname{MIN}\left(\frac{\rho k}{\omega}, \frac{a_{1} \rho k}{\bar{\Omega} F_{2}}\right)
$$

in which

$$
\begin{gathered}
F_{2}=\tanh \left(\arg 2^{2}\right), \quad \arg g_{2}=\operatorname{MAX}\left(2 \frac{\sqrt{k}}{\beta_{k} \omega y}, \frac{500 v}{y^{2} \omega}\right), \\
\bar{\Omega}=\sqrt{2 \Omega: \Omega}, \quad \Omega_{i j}=\frac{1}{2}\left(\frac{\partial u_{i}}{\partial x_{j}}-\frac{\partial u_{j}}{\partial x_{i}}\right),
\end{gathered}
$$

where $a_{1}=0.31$ is the Bradshaw constant. The underlying idea is to remedy the tendency of two-equation models to overestimate the shear stress, in particular for flows with adverse pressure gradients. For this purpose, a bound on the stressintensity ratio $u^{\prime} v^{\prime} / k$ is imposed. The limitation of $v_{t}$ ensures that $u^{\prime} v^{\prime} / k \leqslant a_{1}$ while $a_{1}=0.31$.

\subsection{3 $\gamma-R e_{\theta t}$ transition transport model}

The $\gamma-R e_{\theta t}$ model holds two transport equations, one for the intermittency $\gamma$ and the other for the Reynolds number based on momentum thickness at the transition point, $R e_{\theta t}$. The intermittency $\gamma$ represents the fraction of time the flow is turbulent at a fixed position in the flow field. It is zero in case of laminar flow and one in case of turbulent flow. In the transition region, $\gamma$ varies in between zero and one. The transport equation for $R e_{\theta t}$ is controlled by an empirical transition criterion, which is only valid outside of the boundary layer. Inside the boundary layer, the information about transition onset is transported by convection and diffusion terms.

$$
\begin{gathered}
\frac{\partial(\rho \gamma)}{\partial t}+\nabla \cdot(\rho \boldsymbol{u} \gamma)-\nabla \cdot\left(\left(\mu+\frac{\mu_{t}}{\sigma_{\gamma}}\right) \nabla \gamma\right)=\mathscr{P}_{\gamma}-\mathscr{E}_{\gamma}, \\
\frac{\partial\left(\rho \operatorname{Re}_{\theta t}\right)}{\partial t}+\nabla \cdot\left(\rho \boldsymbol{u} R e_{\theta t}\right)-\nabla \cdot\left(\sigma_{\theta t}\left(\mu+\mu_{t}\right) \nabla R e_{\theta t}\right)=\mathscr{P}_{\theta} t .
\end{gathered}
$$

The production term of the intermittency equation is given by

$$
\mathscr{P}_{\gamma}=F_{\text {length }} c_{a 1} \rho \tilde{S} \sqrt{\gamma F_{\text {onset }}}\left(1-c_{e 1} \gamma\right) \text {, }
$$

where $\tilde{S}$ is the magnitude of the strain rate and $F_{\text {length }}$ is an empirical function that controls the length of the transition area. It is defined by the second transport quantity $R e_{\theta t} . F_{\text {onset }}$ is used to trigger the intermittency production. The empirical 
function $F_{\text {length }}$ is given by

$$
F_{\text {length }}=\left\{\begin{array}{ll}
398.2 e^{-1}-119.3 e^{-4} R e_{\theta t}-132.6 e^{-6} R e_{\theta t}^{2} & \text { if } R e_{\theta t}<400 \\
263.4-124.0 e^{-2} R e_{\theta t}+194.5 e^{-5} R e_{\theta t}^{2}-101.7 e^{-8} R e_{\theta t}^{3} & \text { if } 400 \leq R e_{\theta t}<596 \\
0.5-\left(R e_{\theta t}-596.0\right) \cdot 3.0 e^{-4} & \text { if } 596 \leq R e_{\theta t}<1200 \\
0.319 & \text { if } 1200 \leq R e_{\theta t}
\end{array} .\right.
$$

Additionally, an extra function $F_{\text {sublayer }}$ is introduced which accounts for the right value of $F_{\text {length }}$ in the viscous sublayer downstream the transition point, which is defined by

$$
F_{\text {length }}=F_{\text {length }} \cdot\left(1-F_{\text {sublayer }}\right)+40.0 \cdot F_{\text {sublayer }},
$$

in which,

$$
F_{\text {sublayer }}=\exp \left(-\left(\frac{5}{2} R e_{\omega}\right)^{2}\right)
$$

in which,

$$
R e_{\omega}=\frac{\rho y^{2} \omega}{500 \mu}
$$

The production term of the second transport equation for $R e_{\theta t}$ is given by $F_{\text {onset, }, 1}$. The empirical function $F_{\text {onset }}$ is derived from the three functions $F_{\text {onset }, 1}, F_{\text {onset }, 2}$ and $F_{\text {onset }, 3}$ as

$$
F_{\text {onset }, 1}=\frac{R e_{v}}{2.193 R e_{\theta c}}
$$

in which,

$$
\begin{gathered}
\operatorname{Re}_{\theta c}=\frac{\rho y^{2} \tilde{S}}{\mu}, \\
F_{\text {onset }, 2}=\operatorname{MIN}\left(\operatorname{MAX}\left(F_{\text {onset }, 1}, F_{\text {onset }, 1}^{4}\right), 2.0\right), \\
F_{\text {onset }, 3}=\exp \left(1-\left(\frac{2 \mu_{t}}{5 m u}\right)^{3}\right), \\
F_{\text {onset }}=\operatorname{MAX}\left(F_{\text {onset }, 2}-F_{\text {onset }, 3}, 0.0\right) .
\end{gathered}
$$

Here, the Reynolds number at the transition point is replaced by an empirical function $R e_{\theta c}$, which forces the production of intermittency upstream of the transition point. $R e_{\theta c}$ is given by

$$
F_{\text {length }}=\left\{\begin{array}{lr}
R e_{\theta t}-396 e^{-2}-120.7 e^{-4} R e_{\theta t}+868.2 e^{-6} R e_{\theta t}^{2}-696.5 e^{-9} R e_{\theta t}^{3}+174.1 e^{-12} R e_{\theta t}^{4} \\
R e_{\theta t}-593.1+\left(R e_{\theta t}-1870\right) \cdot 0.482 & \text { if } R e_{\theta t} \leq 1870 \\
& \text { if } 1870<R e_{\theta t}
\end{array}\right.
$$


The destruction or relaminarisation term of the intermittency equation is

$$
\mathscr{E}_{\gamma}=c_{a 2} \rho \tilde{\Omega} \gamma F_{t u r b}\left(c_{e 2} \gamma-1\right),
$$

where $\tilde{\Omega}$ is the vorticity magnitude and $F_{\text {turb }}$ is an empirical function that depends on the viscosity ratio

$$
F_{\text {turb }}=\exp \left(-\left(\frac{1 \mu_{t}}{2 m u}\right)^{4}\right) \text {. }
$$

The constants for the intermittency equation are

$$
c_{e 1}=1.0, \quad c_{a 1}=0.5, \quad c_{e 2}=50.0, \quad c_{a 2}=0.03, \quad \sigma_{\gamma}=1.0 .
$$

The production term of the second transport equation for $R e_{\theta t}$ is given by

$$
\mathscr{P}_{\theta t}=c_{\theta t} \frac{\rho}{t}\left(R e_{\theta t}^{\star}-R e_{\theta t}\right)\left(1.0-F_{\theta t}\right),
$$

where $R e_{\theta t}^{\star}$ is obtained from an empirical transition criterion and $F_{\theta t}$ is an empirical function. The transition criterion was introduced by Langtry and Menter:

$$
F_{\text {length }}=\left\{\begin{array}{ll}
\operatorname{Re}_{\theta t}^{\star}=\left[1173.51-589.43 T u+\frac{0.2196}{T u^{2}}\right] F\left(\gamma_{\theta}\right) & \text { if } T u \leq 1.3 \% \\
\operatorname{Re}_{\theta t}^{\star}=331.5[\mathrm{Tu}-0.5658]^{-0.671} F\left(\gamma_{\theta}\right) & \text { if } 1.3 \%<T u
\end{array},\right.
$$

where

$$
F_{\text {length }}=\left\{\begin{array}{ll}
F\left(\gamma_{\theta}\right)=1-\left[-13 \gamma_{\theta}-123.7 \gamma_{\theta}^{2}-405.7 \gamma_{\theta}^{3}\right] \exp \left(-\left(\frac{2 T u}{3}\right)^{1.5}\right) & \text { if } \gamma_{\theta} \leq 0 \\
F\left(\gamma_{\theta}\right)=1+0.275\left[1-\exp \left(-35 \gamma_{\theta}\right)\right] \exp (-2 T u) & \text { if } 0<\gamma_{\theta}
\end{array} .\right.
$$

For numerical robustness, the following limitations are used:

$$
-0.1 \leq \gamma_{\theta} \leq 0.1, \quad T u \geq 0.027, \quad R e_{\theta t} \geq 20 .
$$

The pressure gradient parameter $\gamma_{\text {thet }}$ and the turbulence intensity $T u$ are defined by

$$
\gamma_{\theta}=\frac{\rho \theta^{2}}{\mu} \frac{d U}{d s}, \quad T u=100 \sqrt{\frac{2 k}{3 U}} .
$$

The velocity gradient $d U / d s$ is the acceleration along the streamwise direction and can be computed by the derivatives of $U$, the coordinates $x, y$ and $z$ and the velocity components $u, v$ and $w$.

The use of streamwise direction is not Galilean invariant. The empirical function $F_{\theta t}$ is

$$
F_{\theta t}=\operatorname{MIN}\left(\operatorname{MAX}\left(F_{\text {wake }} \cdot \exp \left(-\left(\frac{y}{\delta}\right)^{4}\right), 1.0\right),\right.
$$

where $\delta$ is the boundary layer thickness and $F_{\text {wake }}$ depends on the Reynolds number based on $\omega$ as follows

$$
\theta_{B L}=\frac{\operatorname{Re}_{\theta t} \mu}{\rho U}
$$




$$
\begin{gathered}
\delta_{B L}=7.5 \theta_{B L}, \\
\delta=\frac{50 \tilde{\Omega} y}{U} \delta_{B L}, \\
R e_{\omega}=\frac{\rho \omega y^{2}}{\mu}, \\
F_{\text {wake }}=\exp \left(-\left(\frac{R e_{\omega}}{10^{50}}\right)^{2}\right) .
\end{gathered}
$$

The model constants for the $R e_{\theta t}$ transport equation are

$$
c_{\theta t}=0.03, \quad \sigma_{\theta t}=2.0 \text {. }
$$

Finally, the intermittency is modified by prediction of separation induced transition using

$$
\gamma_{\text {sep }}=\operatorname{MIN}\left(s_{1} \operatorname{MAX}\left(0, \frac{R e_{v}}{3.235 \operatorname{Re}_{\theta c}}-1\right) F_{\text {reattach }}, 2.0\right) F_{\theta t}
$$

where $s_{1}=2.0$ and $F_{\text {reattach }}=\exp \left(-\left(\frac{\mu_{t}}{20 \mu}\right) 4\right)$. The effective intermittency becomes $\gamma_{\text {eff }}=\operatorname{MAX}\left(\gamma, \gamma_{\text {eff }}\right)$. The effective intermittency is coupled to the $k$-equation of the Menter-SST turbulence model. Production and destruction terms are modified as follows

$$
\tilde{\mathscr{P}}_{k}=\gamma_{e f f} \mathscr{P}_{k}, \quad \tilde{\mathscr{D}}_{k}=\operatorname{MIN}\left(\operatorname{MAX}\left(\gamma_{e f f}, 0.1\right), 1.0\right) \mathscr{D}_{k}
$$

The original blending function of the Menter-SSt model F_1, orig is modified using

$$
R_{y}=\frac{\rho y \sqrt{k}}{\mu}, \quad F_{3}=\exp \left(-\left(\frac{R_{y}}{120}\right)^{8}\right), \quad F_{1}=\operatorname{MAX}\left(F_{\text {orig }, 1}, F_{3}\right)
$$

For the two empirical functions $F_{\text {length }}$ and $R e_{\theta c}$, additional approaches exist in the literature and some of them are implemented in DLR-TAU code. The correlations given by Suluksna et al. [TAU technical doc 83] are

$$
\begin{gathered}
F_{\text {length }}=\operatorname{MIN}\left(0,1 \exp \left(-0.022 R e_{\theta t}+12\right)+0.45,300\right), \\
\operatorname{Re}_{\theta c}=\operatorname{MIN}\left(\operatorname{MAX}\left(-\left(0.025 R e_{\theta t}\right)^{2}+1.47 R e_{\theta t}-120,125\right), \operatorname{Re}_{\theta t}\right) .
\end{gathered}
$$

The correlations of Pettersson et al. [TAU technical doc 70] are

$$
\begin{gathered}
F_{\text {length }}=0.086743+75.285 \exp \left(-\frac{\left(R e_{\theta t}-43.789\right)^{2}}{50313},\right. \\
R e_{\theta c}=0.92345 R e_{\theta t} .
\end{gathered}
$$

The correlations of Krause et al. [TAU technical doc 45] are

$$
F_{\text {length }}=\left\{\begin{array}{ll}
\frac{\ln \left(R e_{\theta t}+1\right)}{T u_{\infty}} & \text { if } T u_{\infty} \leq 1.0 \% \\
\ln \left(R e_{\theta t}+1\right)\left(0.2337 T u_{\infty}^{2}-1.3493 T u_{\infty}+2.1449\right) & \text { if } 1.0 \%<T u_{-} \infty
\end{array},\right.
$$




$$
R e_{\theta c}=\frac{R e_{\theta t}}{-0.042 T u_{\infty}^{3}+0.4233 T u_{\infty}^{2}+0.0118 T u_{\infty}^{2}+1.0744} .
$$

Finally, the correlations of Malan et al. [TAU technical doc 52] are

$$
\begin{gathered}
F_{\text {length }}=\operatorname{MIN}\left(\exp \left(7.168-0.01173 R e_{\theta t}\right)+0.5,300\right), \\
\operatorname{Re}_{\theta c}=\operatorname{MIN}\left(0.615 R e_{\theta t}+61.5, R e_{\theta t}\right) .
\end{gathered}
$$

In addition to the transition criterion given by Menter and Langtry, the transition criterion of Abu-Ghannam and Shaw [TAU technical doc 4] is implemented using the local turbulence intensity, instead of the freestream turbulence intensity, which is

$$
R e_{\theta t}^{\star}=163+\exp \left(F\left(\gamma_{\theta}\right)-\frac{F \gamma_{\theta}}{6.91} \mathrm{Tu}\right)
$$

where

$$
F_{\text {length }}= \begin{cases}F\left(\gamma_{\theta}\right)=6.91+12.75 \gamma_{\theta}+63.64 \gamma_{\theta}^{2} & \text { if } \gamma_{\theta}<0 \\ F\left(\gamma_{\theta}\right)=6.91+2.48 \gamma_{\theta}-12.27 \gamma_{\theta}^{2} & \text { if } \gamma_{\theta}>0\end{cases}
$$

\subsection{Spatial discretisation}

Spatial discretisation is used to calculate the viscous and inviscid fluxes on a cell through numerical approximation. The major methods of spatial discretisation are finite differential method (FDM), finite volume method (FVM) and finite element method (FEM). The finite volume method is capable of better ensuring the conservation laws, and is easier implemented in more complex domains[111]. In this section, upwind scheme and central scheme will be discussed.

\subsubsection{Upwind scheme}

The approximation of the governing equations can be done with finite-difference quatients. The partial differential equations involves a number of different partial derivative terms which can be replaced by finite differences. The resulting formula is call a difference equation. This difference equation is an algebraic representation of the partial differential equation. To be more detailed, we consider the the continuity equation

$$
\frac{\partial \rho}{\partial t}+\frac{\partial(\rho u)}{\partial x}=0
$$

If we apply the velocity $u$ as constant, equation 2.4 takes the typical form of a linear convection equation:

$$
\frac{\partial \rho}{\partial t}+u \frac{\partial \rho}{\partial x}=0, \quad \text { or } \quad \rho_{t}+u \rho_{x}=0
$$

which decribes the transport of mass $\rho$ by a flow of velocity $u$. 
Taking the flux balance for the dual cell around point $i$, a superscript $n$ denote the time step and applying a forward difference scheme in time, the corresponding finite volume reads

$$
\frac{\rho_{i}^{n+1}-\rho_{i}^{n}}{\Delta t}=-\frac{u}{\Delta x}\left(\rho_{i+\frac{1}{2}}^{n}-\rho_{i-\frac{1}{2}}^{n}\right) .
$$

As $u>0$ and constant we may use a simple upwind scheme, i.e. the flux at $i+\frac{1}{2}$ and $i-\frac{1}{2}$ is approximated by $\rho_{i}$ and $\rho_{i-1}$, respectively, then we have

$$
\frac{\rho_{i}^{n+1}-\rho_{i}^{n}}{\Delta t}=-\frac{u}{\Delta x}\left(\rho_{i}^{n}-\rho_{i-1}^{n}\right)
$$

which represents a first-order upwind scheme.

This upwind scheme can be written in artificial viscosity form, i.e., as a central scheme with artificial dissipation. If we use a central scheme for the flux at $i+\frac{1}{2}$ and $i-\frac{1}{2}$, then we have

$$
\frac{\rho_{i}^{n+1}-\rho_{i}^{n}}{\Delta t}=-\frac{u}{\Delta x}\left[\frac{1}{2}\left(\rho_{i}^{n}+\rho_{i+1}^{n}\right)-\frac{1}{2}\left(\rho_{i-1}^{n}+\rho_{i}^{n}\right)\right]
$$

which is of second order accuracy. Subtracting the upwind scheme as in equation 2.82 from the central scheme as in equation 2.83 , we get

$$
\left(\rho_{i}^{n}-\rho_{i-1}^{n}\right)-\left[\frac{1}{2}\left(\rho_{i}^{n}+\rho_{i+1}^{n}\right)-\frac{1}{2}\left(\rho_{i-1}^{n}+\rho_{i}^{n}\right)\right]=-f \operatorname{rac} 12\left(\rho_{i}^{n}-\rho_{i+1}^{n}\right)+\frac{1}{2}\left(\rho_{i-1}^{n}-\rho_{i}^{n}\right) .
$$

Thus the upwind scheme can be written as

$\frac{\rho_{i}^{n+1}-\rho_{i}^{n}}{\Delta t}=-\frac{u}{\Delta x}\left[\frac{1}{2}\left(\rho_{i}^{n}+\rho_{i+1}^{n}\right)-\frac{1}{2}\left(\rho_{i-1}^{n}+\rho_{i}^{n}\right)-f \operatorname{rac} 12\left(\rho_{i}^{n}-\rho_{i+1}^{n}\right)+\frac{1}{2}\left(\rho_{i-1}^{n}-\rho_{i}^{n}\right)\right]$.

Hence, the upwind scheme can be written in artificial viscosity form as

$$
\begin{aligned}
\frac{\rho_{i}^{n+1}-\rho_{i}^{n}}{\Delta t}= & -\frac{u}{\Delta x}\left\{\left[\frac{1}{2}\left(\rho_{i}^{n}+\rho_{i+1}^{n}\right)-\frac{1}{2}\left(\rho_{i-1}^{n}+\rho_{i}^{n}\right)\right]\right. \\
& \left.-\left[\frac{1}{2}\left(\rho_{i}^{n}-\rho_{i+1}^{n}\right)-\frac{1}{2}\left(\rho_{i-1}^{n}-\rho_{i}^{n}\right)\right]\right\}
\end{aligned}
$$

\subsubsection{Central scheme}

Same as the explanation in upwind scheme in the former section, the approximation of the governing equations is again done with finite-difference quotients. we take the continuity equation 2.79 and equation 2.80 , and apply again the appropriate initial and boundary conditions and $u>0$, this becomes a first order hyperbolic 
equation. Using a second order difference formula for the discretisation of the spatial derivative $\rho_{x}$ at mesh point $i$ and again a forward difference formula for the time derivative, the discrete equation read:

$$
\frac{\rho_{i}^{n+1}-\rho_{i}^{n}}{\Delta t}=-\frac{u}{\Delta x}\left(\rho_{i+1}^{n}-\rho_{i-1}^{n}\right)
$$

The index $n$ describes the time level. We now have a second-order central scheme.

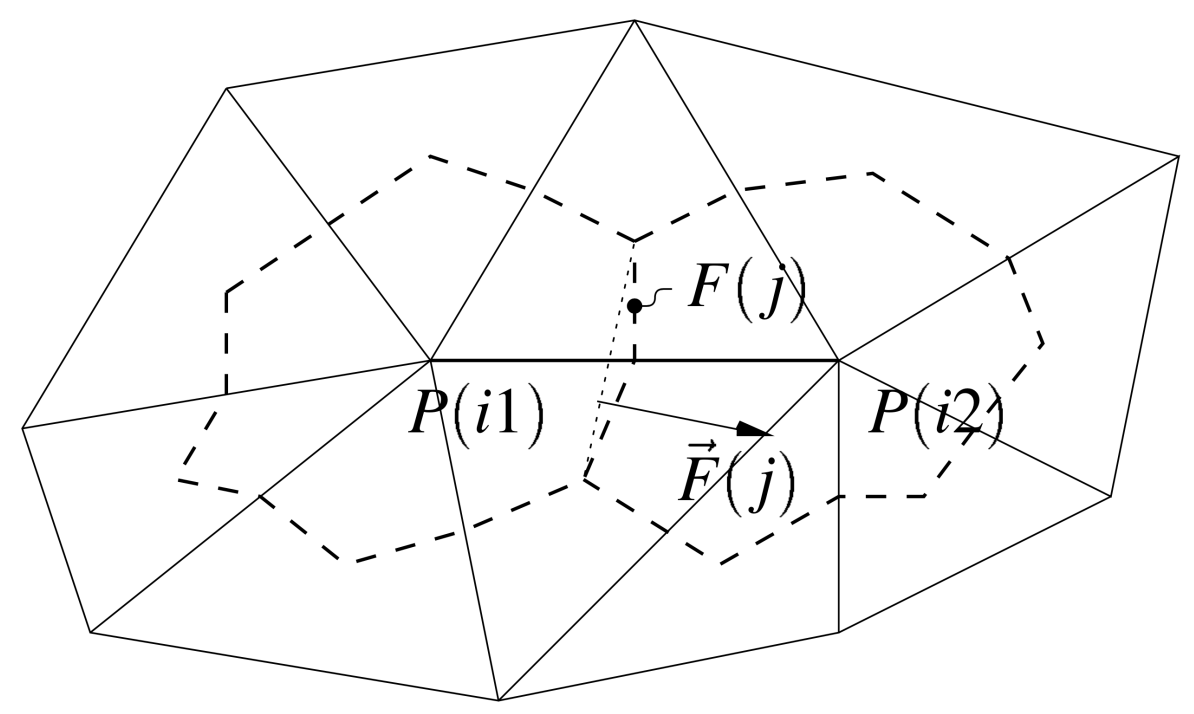

Figure 2.1: Control volumes around neighboring points.

As shown in figure 2.1, let the points $P(j 1)$ and $P(j 2)$ be separated by the face $F(i): P(j 1)=C_{F}^{P}(i, 1)$ and $F(i): P(j 2)=C_{F}^{P}(i, 2)$. The convective fluxes over the face are computed based on the flow conditions in the points $P(j 1)$ and $P(j 2)$. The convective fluxes have to be considered as fluxes between the control volumes surrounding the points $P(j 1)$ and $P(j 2)$. The size and orientation of the face is described by the face noremal vector $\vec{F}_{i}=\left(F_{i}^{x}, F_{i}^{y}, F_{i}^{z}\right)^{T}$ as shown in figure 2.1. The central fluxes over the face can then be computed as

$$
\vec{Q}_{F}^{F, c}=\frac{1}{2}\left(\overrightarrow{F_{r}}(i)+\overrightarrow{F_{l}}(i)\right)-\frac{1}{2} \tilde{\alpha}\left(\overrightarrow{\omega_{r}}-\overrightarrow{\omega_{l}}\right),
$$

in which, $\tilde{\alpha}$ describes the type of the dissipation, scalar or matrix dissipation. The difference $\overrightarrow{\omega_{r}}+\overrightarrow{\omega_{l}}=\vec{D}(j 1)$ is computed as:

$$
\vec{D}(j 1)=\left(\overrightarrow{\omega_{r}}-\overrightarrow{\omega_{l}}\right)=\varepsilon_{(2)}\left(\overrightarrow{u_{r}}-\overrightarrow{u_{l}}\right)-\varepsilon_{(4)}\left[L\left(\overrightarrow{u_{r}}\right)-L\left(\overrightarrow{u_{l}}\right)\right]
$$

The vector $\vec{w}$ holds the conservative variables $\rho, \rho u, \rho v, \rho w$ and $\rho E$. The Laplacian $\nabla^{2}$ of $L\left(\overrightarrow{u_{i}}\right)$ is calculated from each neighboring point

$$
L\left(\overrightarrow{u_{i}}\right)=\sum_{j=1}^{n \text { neighbors }}\left(\overrightarrow{u_{j}}-\overrightarrow{u_{i}}\right)
$$




\subsection{Temporal discretisation}

Once the spatial discretisation of the system has been done, the system of the PDEs can be rewritten in a discrete form as:

$$
\frac{d u}{d t}=F(u)
$$

in which, $u$ is a time-dependent $\mathrm{N}$-dimension vector, originates from the spatial discretisation of the computational domain $D$, and $F$ is the numerical discretisation of the fluxes on the same domain. The temporal discretisation is then employed in calculating the evolution in time of the system Eq. 2.91, once a solution at the initial time $t_{0}$ (initial value problem) is given. By performing the temporal discretisation, a set of snapshots fo the solution is obtained. These snapshots represent the solution of the unknown function at consecutive instants.

$$
\left\{u\left(t_{0}\right), u\left(t_{1}\right), u\left(t_{2}\right), \quad \ldots\right\}
$$

Numerical methods used for the temporal discretisation of equations 2.92 are mainly devided into two categories: implicit and explicit schemes. In implicit methods, the function at the current step $t_{n}$ depends on the current step $t_{n}$ itself. Explicit methods refer to all the schemes at the current step (i.e. using the solutions at time $\left.t_{n-1}, t_{n-2}\right)$. It is possible to obtain the solution at the time $t_{n}$. Explicit methods are commonly easy to implement, but they are limited in obtaining a stable solution. Meanwhile, implicit methods are more stable, but lead to high computational cost since they require inverting the function used to describe the time evolution, which is defined by the specific schemes. Within all the possible numerical methods for the numerical integration, the explicit and implicit Euler methods are the simplest choices [91].

\subsection{Multigrid technique}

Multigrid technique is a very effective method for speeding up the convergence by a considerable scale[8]. Finer grid levels could very efficiently eliminate the high frequency error compared to low frequency error. However, a coarser mesh is capable of eliminating low frequency error. Taking advantage of the capability of both coarse and fine mesh in eliminating high and low frequency error, multigrid technique could speed up the convergence. Furthermore, coarse meshes take comparatively less time to converge, which also reduces the computational time.

Since the multigrid does not depend on the equations to be solved, we can consider a scalar non-linear equation:

$$
L(w)=S,
$$


with the solution $w$ and a solution independent right hand side $S$. With the finest grid $f$, the equation is discretised as a system:

$$
L_{f}\left(w_{f}\right)=S_{f},
$$

which has to be solved simultaneously for all control volumes. The true solution $w_{f}^{\text {true }}$ of the discretised equation is iterated numerically. Each iteration step starts with a solution $w_{f}^{0}$ and ends wigh $w_{f}^{1}$. The normal convergence bebaviour of an iteration method slows down after a rapid start, due to the fact that low frequency errors are hardly dampened. The advantage of a multigrid method is that these low frequency errors (attached to a long wavelength) can be well resolved on a coarser mesh. On a coarser mesh, the calculation of these errors is much easier because its wavelength becomes shorter compared to the grid spacing and the number of equations to be solved decreases with the number of agglomerated volumes. In addition, the higher order terms of an upwind scheme can be neglected, because they are not necessary to resolve the dampening of a smooth low frequency error. But the equations on the coarser grid have to be changed in a way that the solution converges to the fine grid solution. Therefore, an iteration step on the fine grid results in a residual res:

$$
r e s_{f}=L_{f}\left(w_{f}^{1}\right)-S_{f} .
$$

By subtracting from the true solution, we get

$$
L_{f}\left(w_{f}^{\text {true }}\right)-L_{f}\left(w_{f}^{1}\right)=-r e s_{f} .
$$

For the smooth low frequency errors this leads on a coarser mesh $(\mathrm{m})$ to

$$
L_{m}\left(w_{m}^{\text {true }}\right)=I_{f m}^{r}\left(-r e s_{f}\right)+L_{m}\left(w_{m}^{0}\right) .
$$

The restriction operator $I^{r}$ of the residual has to obey

$$
I_{f m}^{r}\left(-r e f_{f}\right)=-I_{f m}^{r}\left(-r e f_{f}\right) \Rightarrow I_{f m}^{r}(0)=0 .
$$

If the residual on the fine grid equals to zero, the starting solution is a true solution of equation 2.97. As a starting value for the iteration on the mesh $m$, a restriction $I^{w}$ of the iterated fine grid solution is used:

$$
w_{m}^{0}=I_{f m}^{w}\left(w_{f}^{1}\right) \text {. }
$$

The difference between the best approximation $\left(w^{b a}\right)$ of the true solution and the starting solution is called correction $C$ :

$$
C_{m}=w_{m}^{b a}-w_{m}^{0}=w_{m}^{b a}-I_{f m}^{w}\left(w_{f}^{1}\right) .
$$

The correction is prolongated to the finer grid to improve the solution there

$$
w_{f}^{b a}=w_{f}^{1}+I_{m f}^{c}\left(C_{m}\right) .
$$


The same approach can be applied to calculate the best approximation to the true solution of equation 2.97 on the grid $m$ by considering an even coarser mesh $c$. An iteration step on grid $m$ stops with a residual

$$
r e s_{m}=L_{m}\left(w_{m}^{1}\right)-L_{m}\left(w_{m}^{0}\right)-I_{f m}^{r}\left(-r e s_{f}\right),
$$

which can be used to formulate equation 2.97 on the next coarser grid $(c)$ :

$$
L_{c}\left(w_{c}^{\text {true }}\right)=I_{m c}^{r}\left(-\operatorname{res}_{m}\right)+L_{c}\left(w_{c}^{0}\right) .
$$

The way to a coarser mesh can be taken again and again, but in practice, the use of more than about four mehses does not increase the convergence sufficiently to pay for the additional operations. On the coarsest grid the iterated solution is taken as the best approximation.

Due to the different equations on the fine grid and on the coarser ones the residuals res are defined differently depending on the grid level. In a different notation these residuals $R$ of the governing equation. The equation on the coarser level 2.97 then gives

$$
\frac{d}{d t} w_{m}+R_{m}-F_{m}=0
$$

with a forcing function $F_{m}$ given by

$$
F_{m}=R_{m}\left(w_{m}^{0}\right)-I_{f m}^{r}\left(R_{f}\left(w_{f}^{1}\right)-F_{f}\right) .
$$

The forcing function on the finest grid is equal to zero. 


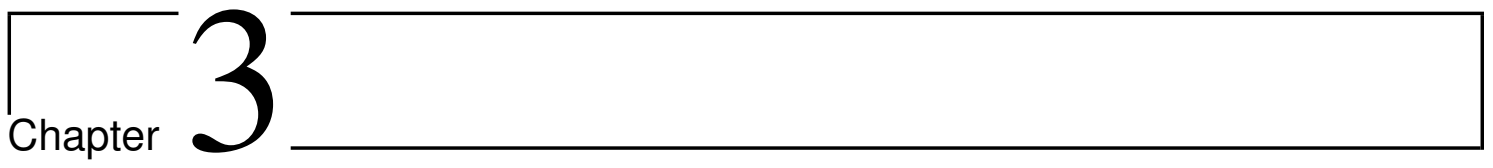

\section{Stability Analysis and Sensitivity Analysis}

\section{Contents}

3.1 Stability analysis . . . . . . . . . . . . . . . . 24

3.1 .1 Linear stability theory $\ldots \ldots \ldots \ldots \ldots \ldots$

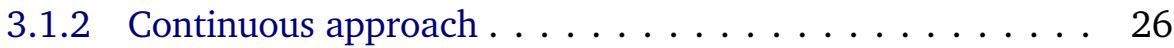

3.1 .3 Discrete approach . . . . . . . . . . . . . . 27

3.1.4 Numerical solution of the eigenvalue problem . . . . . . . 29

3.2 Adjoint global eigenvalue problem . . . . . . . . . 31

3.3 Structural sensitivity analysis $\ldots \ldots \ldots \ldots \ldots$

In this chapter, an introduction to global stability analysis is given. With the aid of the definition of the BiGlobal linear stability, the formulation of the related EigenValue Problem (EVP) is given. Furthermore, adjoint formulation and structural sensitivity analysis method are discussed. More detailed reviews of the concepts introduced here can be found in Ref. [2, 10, 18, 31, 33, 54, 55, 74, 85, 104, 105]. Furthermore, the continuous and discret approaches to analyse the stability problem will be introduced in this section.

\subsection{Stability analysis}

\subsubsection{Linear stability theory}

Linear stability analysis is related with the docomposition of any flow quantity $U$ in a base steady flow $\bar{U}$ plus a disturbance component $\tilde{U}$,

$$
U(X, t)=\bar{U}(X)+\varepsilon \tilde{U}(X, t)
$$


This decomposition is fed into the NS equations. After subtracting the equation for the base flow $(O(1)$ terms, corresponding to the base flow) and neglecting the non-linear $O\left(\varepsilon^{2}\right)$ terms, the perturbed problem can be stated only in $O(\varepsilon)$ terms.

Particularly, in the case of the incompressible NS equations $U=(u, P)$ yields:

$$
\begin{aligned}
\frac{\partial \tilde{u}}{\partial t}+(\tilde{u} \cdot \nabla) \bar{u}+(\bar{u} \cdot \nabla) \tilde{u} & =\frac{1}{R e} \nabla^{2}-\nabla \tilde{P}, \\
\nabla \cdot \tilde{u} & =0 .
\end{aligned}
$$

In the modal stability theory, using separation of variables, the general solution can be found as a linear superposition of normal modes, each of the form

$$
\tilde{U}(X, t)=\hat{U}(X) \exp (\Omega t)
$$

When this decomposition is introduced into the linearised problem the stability analysis becomes a generalised engenvalue problem (EVP) of the form, as an example, this decomposition and the definitions of matrices $A$ and $M$, are given below as

$$
A \hat{U}=\Omega M \hat{U} .
$$

Using this approach, the stability of the base flow is determined by the eigenvalues of the EVP. If $\operatorname{Re}(\Omega)<0$ for all the eigenvalues, the base flow is asymptotically stable and unstable if $\operatorname{Re}(\Omega)>0$ for at least one eigenvalue.

In the NS solutions, depending on the non-homogeneities of the flow, a wide range of simplifications to the flow stability problem are possible in order to make it solvable. From the simplest parallel-flow assumption to the TriGlobal instability analysis complexity increases for both the base flow and disturbances. In the following, a general review of the instability problem formulation for increasing flow complexity are given.

Orr-Sommerfeld approach[76]: Local stability analysis is considered in the study of fluid problems with only one inhomogeneous direction. Despite the fact that almost any real flow of interest is truly inhomogeneous, its simplicity and its low computational cost have made this approach one of the most used tools to study stability of many flows: shear layers, boundary layer transition, jet flows and even secondary instabilities.

The local stability approach relies on the parallel flow assumption. The base flow depends only on the inhomogeneous direction (usually spanwise direction, $y)$.

This is the standard approach in boundary layer transition problem or when curvature effects are negligible. In these cases, the decomposition is considered as $[92,96]$ :

$$
U(x, y, z, t)=\bar{U}(y)+\epsilon \hat{U}(y) e^{\theta_{1 D}}, \quad \theta_{1 D}=\alpha x+\beta z-\Omega t,
$$

permitting the periodic evolution in the spanwise $(\beta)$ and streamwise $(\alpha)$ direction and temporal $(\Omega)$ perturbations. 
Parabolised Stability Equations (PSE). This is considered an extension of the local-stability problem when small non-local parallel effects are taking[43]

$$
U(x, y, z, t)=\bar{U}\left(x^{*}, y\right)+\epsilon \hat{U}\left(x^{*}, y\right) e^{\theta_{2 D}^{*}}, \quad \theta_{2 D}^{*}=\int_{x_{0}}^{x} \alpha(\xi) d \xi+\beta z-\Omega t,
$$

where, $x^{*}=\epsilon x$, and $\epsilon \simeq$ boundary layer thickness accounts for small non-parallel flows in the streamwise direction. The resulting linear system is parabolic in $x^{*}$ and measure the evolution of an initial unsteady (or stable) mode obtained at the initial position $x_{0}$ by local analysis. Non-linear terms can also be retaining to obtain the non-linear PSE.

BiGlobal stability analysis assumes spatial homogeneity in only one out of three spatial directions. The other two directions being resolved in a coupled manner. Consequently, this approach is appropriate for analysis of the temporal or spatial development of small-amplitude (modal or non-modal[93, 96]perturbations, superimposed upon steady or time-periodic two-dimensional laminar or turbulent basic states over complex geometries. Such states may comprise one, two or all three velocity components, all of which may be two-dimensional functions of the resolved coordinates[104].

$$
U(x, y, z, t)=\bar{U}(x, y)+\epsilon \hat{U}(x, y) e^{\theta_{2 D}}, \quad \theta_{2 D}=\beta z-\Omega t,
$$

substituting this approach into the NS equations results in an eigenvalue problem for the determinsation fo the complex eigenvalue $\Omega+\Omega_{r}+i \Omega_{i}$. In the temporal framework used, $\Omega_{r}$ represents the amplification/damping rate and $\Omega_{i}$ is a frequency of the disturbance sought, while quantities with bars and hats denote base and disturbance flow quantities, respectively. Spatial homogeneity along the direction $z$ is assumed, in which the wavenumber parameter $\beta$ is introduced, related with a periodicity length $L_{z}$ along this direction by $L_{z}=2 \pi / \beta$.

TriGlobal stability analysis is the natural extension of BiGlobal stability analysis to a fully three-dimensional base flow and its perturbation. TriGlobal does not assume any homogeneity either in the flow or the perturbation,

$$
U(x, y, z, t)=\bar{U}(x, y, z)+\epsilon \hat{U}(x, y, z) e^{\theta_{3 D}^{*}}, \quad \theta_{3 D}=\Omega t .
$$

With this approach, an eigenvalue problem of a linear system of three-dimensional partial differential equations is obtained. Only the parameter $\Omega$ is involved in the computation. This solution, although is capable of providing more information than any other approach, is by far the most expensive computationally. The eigenvalue problem is computationally very demanding. Only a few works can be found in the literature to the application of TriGlobal analysis to fluid dynamics[53]. This is the main reason why other alternatives, such as the time stepping methods[108], are considered in the analysis of 3D flows.

There are two approaches solving the stability problem, one is the continuous approach and the other is discrete approach, which will be discussed in the following sections. 


\subsubsection{Continuous approach}

In the continuous approach, the stability equations are derived directly from the NS equations and then liearised to form the continuous operator. In this context, the method in the BiGlobal analysis for the incompressible NS equations will be illustrated.

Between the simplified paralle-flow assumption and the numerically challenging TriGlobal instability analysis, BiGlobal instability analysis represents an intermediate and affordable step in the instability analysis. The key difference with the parallel flow assumption is that, three-dimensional space comprises an inhomogeneous two-dimensional domain which is extended periodically in $z$ characterised by a wavelength $L_{z}$. Considering the decomposition exposed in equation 3.7, substituted into the linearised equations equation 3.2 yields:

$$
\begin{aligned}
{\left[\mathscr{L}-\left(\mathscr{D}_{x} \bar{u}\right)\right] \hat{u}-\left(\mathscr{D}_{y} \bar{u}\right) \hat{v}-\mathscr{D}_{x} \hat{p} } & =-i \Omega \hat{u}, \\
-\left(\mathscr{D}_{x} \bar{v}\right) \hat{u}+\left[\mathscr{L}-\left(\mathscr{D}_{y} \bar{v}\right)\right] \hat{v}-\mathscr{D}_{y} \hat{p} & =-i \Omega \hat{v}, \\
-\left(\mathscr{D}_{x} \bar{w}\right)-\left(\mathscr{D}_{y} \bar{w}\right) \hat{v}+\mathscr{L} \hat{w}-i \beta \hat{p} & =i \Omega \hat{w}, \\
\mathscr{D}_{x} \hat{u}+\mathscr{D}_{y} \hat{v}+i \beta \hat{w} & =0,
\end{aligned}
$$

where $\mathscr{D}_{x}=\partial / \partial x, \mathscr{D}_{y}=\partial / \partial y$ and

$$
\mathscr{L}=\frac{1}{R e}\left(\frac{\partial^{2}}{\partial x^{2}}+\frac{\partial^{2}}{\partial y^{2}}-\beta^{2}\right)-\bar{u} \frac{\partial}{\partial x}-\bar{v} \frac{\partial}{\partial y}-i \beta \bar{w} .
$$

The boundary conditions depend on the particular problem to analyse but are usually considered homogeneous either in the value of the perturbation or in its normal derivative. Only the particular case of PSE, which more than a real eigenvalue is an evolution problem in the streamwise direction, a Dirichlet boundary condition is imposed at the beginning of the domain. This boundary (initial) condition is usually obtained by the most unstable modes solution of the Orr-Sommerfeld equation and its streamwise evolution is captured by the PSE.

Moreover, in the strong fromulation, continuity between the function and its derivative in the direction normal to the interface is imposed at the interface for each flow variable $U$. Namely, considering the interface between domains 1 and 2 yields:

$$
\begin{aligned}
U_{N_{x}, i}^{1} & =U_{0, i}^{2}, \\
\left.\frac{\partial U^{1}}{\partial x}\right|_{N_{x}, i} & =\left.\frac{\partial U^{2}}{\partial x}\right|_{0, i} .
\end{aligned}
$$

For the particular case of non-conforming grids, the interpolation tool is necessary fro imposing interface conditions. For example, supposing connection between domains in $y_{\max }^{1}$ to $y_{\text {min }}^{2}$

$$
\begin{aligned}
U_{j, N_{y}}^{1} & =\stackrel{2 \rightarrow 1}{I_{j, i}} U_{i, 0}^{2}, \\
\left.\stackrel{1 \rightarrow 2}{I_{j, i}} \frac{\partial U^{1}}{\partial y}\right|_{i, N_{y}} & =\left.\frac{\partial U^{2}}{\partial y}\right|_{j, 0} .
\end{aligned}
$$


Assembling all the unknows and equations in one matrix, a generalised eigenvalue problem is finally obtained for the determination of the perturbation eigenvector $\hat{U}=(\hat{u}, \hat{v}, \hat{w}, \hat{p})^{T}$.

$$
A \hat{U}=\Omega M \hat{U}
$$

The matrix $A$ is the equivalent discretised operator of equation 3.9, some details about the topological structure of this matrix are given in the following sections. The matrix $M$ is diagonal, and in general the identity matrix. However, for the incompressible NS equations or when the boundary conditions are implemented in a strong way, some of its elements can be zero, which in any case, does not invalidate the analysis here described.

\subsubsection{Discrete approach}

The discrete approach, valid for any kind of numerical approximations since the starting point is the discretised non-linear PDE. In the discrete approach, we consider a discretisation of a system of PDEs, typically NS equations, in a computational mesh $(h)$ defined over the problem geometry. The discrete approach formulation is independent of the particular numerical scheme used to dicretise the spatial derivatives of the equations. Thus,

$$
M \frac{d U^{h}}{d t}=F\left(U^{h}\right),
$$

in which $U^{h}$ is a vector of dimension $N$, which contains the number of degrees of freedom for the problem. $M$ is usually a mass matrix, and $F\left(U^{h}\right)$ is a vector of dimension $N$, which defines the numerical fluxes obtained by the discretisation of the continous PDEs in the computational mesh. From here, the superscript ${ }^{h}$ is omitted to simplify the notation.

Inserting the decomposition $U(t)=\overline{(} U)+\varepsilon \tilde{U}(t)$ into equation 3.14 and taking Taylor series about the base flow for the numerical fluxes and neglecting second order $(\tilde{U})^{2}$ terms, we get

$$
M \frac{d \tilde{U}}{d t}=\left.\frac{\partial F(U)}{\partial(U)}\right|_{\bar{U}} \tilde{U}
$$

in which, it is assumed that a base flow or steady solution $\bar{U}$ that statisfies $F(\bar{U})=0$ has been computed and converged.

Then we consider these perturbations in the form of normal modes $\tilde{U}=\hat{U} e^{\Omega t}$, which when inserted into equation 3.15, gives an eigenvalue problem equivalent to the one obtained in the continuous approach:

$$
\Omega M \hat{U}=\left.\frac{\partial F(U)}{\partial(U)}\right|_{\bar{U}} \hat{U}=A(\bar{U}) \hat{U}
$$

where $A(\bar{U})=\left.\frac{\partial F(U)}{\partial(U)}\right|_{\bar{U}}$ is referred to as the Jacobian matrix and $\hat{U}$ is the direct eigenmode correspondent to the eigenvalue $\Omega$. 
The Jacobian matrix can be obtained following a discrete approach. The NS equations are first discretised, equation 3.14, and then linearised around a base flow, equation 3.15. This approach does not require the implementation of a new solver specifically developed for the linear Navier-Stokes equations, and in theory, any existing NS solvers can be used for the computation. A practical implementation is to obtain the Jacobian matrix by numerically differentiating the numerical fluxes around the base flow by using a finite difference approximation. Using Taylor series,

$$
F(\bar{U}+\epsilon k)=F(\bar{U})+\left.\epsilon \frac{\partial F(\bar{U})}{\partial q}\right|_{\bar{U}} k+\mathscr{O}\left(\epsilon^{2}\right),
$$

in which, $k$ is a numerical disturbance (no physical relevance) used to evaluate the Jacobian and $\epsilon$ is a relatively small number. neglecting $\epsilon^{2}$ and higher order terms we get:

$$
\frac{F(\bar{U}+\epsilon k)-F(\bar{U})}{\epsilon}=\left.\frac{\partial F(\bar{U})}{\partial U}\right|_{\bar{U}} k \approx A(\bar{U}) k
$$

From a practical implementation point of view, to calculate the $j^{\text {th }}$ column of the Jacobian matrix, the $j_{t h}$ row of the $k$ vector is set to one while the other rows are set to zero. This process is successively repeated for as many number of degrees of freedom of the matrix. The first order formula, equation 3.18, requires the computation of $N$ numerical fluxes, with $N$ being the dimension of $\bar{U}$. As the dimensional of the problem $N$ increases, the computational cost associated to obtain the Jacobian can become prohibitive. However, the sparse nature of most of the numerical schemes introduce different alternatives to reduce the computational cost of this loop, such as evaluating the fluxes which are only part of the stencil of each computational node. High-order spectral multidomain methods, for the natural compactness of their stencil, makes them particularly well suited for this approach. Moreover, second or third order difference schemes can improve the accuracy of the numerical Jacobian but at the expense of additional computation of fluxes. An alternative to improve the accuracy of the Jacobian without sacrificing the computational cost, is to use complex variables[10].

\subsubsection{Numerical solution of the eigenvalue problem}

The stability analysis starts with a NS solver to obtain the base steady state. Once the base steady state is obtained, it is then fed to the linearised flow equations leading to a generalised eigenvalue problem (EVP) for the recovery of the disturbance eigenvectors.

The generalised eigenvalue problem, as in equations 3.13 and 3.17, in in general real and non-symmetric, so specific algorithms must be employed to recover its eigenvalues. Moreover the size and CPU time required to address interesting applications are determinant factors when choosing the EVP solver. In this section, direct and iterative methods will be discussed. 
Embracing the whole numerical analysis, an important feature that compels an specific methodology is the sparsity of the matrices involved. This sparsity pattern may be exploited by making use of specialised formats for storing and handling the data. The choice of a particular format is made according to its compatibility with software package libraries employed for parallel solution of linear systems and EVP solvers.

\subsubsection{Direct methods}

For matrices of small dimension, direct algorithms, as the classic QZ, can recover the whole eigenspectrum. The QZ algorithm is an analog of the QR algorithm for the generalised eigenvalue problem. QR algorithm is based on decompsing the matrix A in a product of an oorthogonal matrix and an upper trangular matrix, $A=Q R$. In the QZ algorithm, the matrix $A M^{-1}$ is QR-decomposed, and the eigenvalues are recovered after an iterative process.

The QZ algorithm hase been implemented in LAPACK (Linear Algebra PACKage). LAPACK is a library in Fortran77 subroutines for solving the most commonly occurring problems in numerical linear algebra. The subroutine xGGEV computes the generalised EVP for $A$ and $M$ non-symmetric matrices.

The QZ algorithm leads to all eigenvalues and, optionally, to the right and left eigenvectors. It requires $\mathscr{O}\left(n^{3}\right)$ memory locations, where $n$ is the order of $A$ and $M$. More specifically, it requires about $30 n^{3}$ floating point operations for computing the eigenvalues only. If right eigenvectors are desired, then an additional $16 n^{3}$ are necessary, and likewise for the left eigenvectors. These estimates of work are based on the experience that about two QZ iterations per eigenvalue is sufficient (the convergence properties of QZ are about the same as for $\mathrm{QR}$ ).

There are two main drawbacks of this methodology: the first one is related with the library itself, LAPACK does not manage sparse format, so storage capabilities are an important limitation for the problem discretization; the second one is due to the algorithm itself, the classic QZ algorithm is inefficient or impractical. The computational resources demanded may constrain the analysis to significant low Reynolds numbers and low dimensional problems.

\subsubsection{Iterative methods}

The major challenge associated with the instability analysis is the size of this generilised non-symmetric matrix eigenvalue problem. High Reynolds number problems require a fine spatial descretisation to reach an accurate solution. The Arnoldi method provides an accurate approximation to an arbitrarily large scope of eigenvalues of a matrix at a fraction of both the memory and the computing time requirements of the QZ algorithm. Arnoldi's procedure starts finding an orthogonal basis in a krylov subspace. Then the matrix $A$ is projected onto this Krylov subspace. The eigenvalues of the orthogonal projection are called the Ritz eigenvalues and 
converge to eigenvalues of $A$. Because the standard Arnoldi methods converge to the eigenvalues with larger real part (in module), in order to be applied to stability analysis, it should be accompanied with different strategies to improve the accuracy of the computation in only a portion of the spectrum, normally the area of real part close to zero: shift-and-invert, Cayley transform, Chebyshev polynomial filtering, least squares or Jacobi-Davidson approach[88] are between the common approaches to get this objective. From them, perhaps the most normally used is the shift-and-invert strategy, which recovers only the part of the eigenspectrum which contains the most interesting, from a physical point of view, unstable/least stable eigenvalues.

If $(\tilde{U}, \Omega)$ is a solution of the generalised EVP, $A \tilde{U}=\Omega M \tilde{U}$, then this strategy solves instead the following

$$
(A-\sigma M)^{-1} M \tilde{U}=\tilde{U},
$$

where $\lambda=\frac{1}{\Omega-\sigma}$, with $\sigma$ a shift parameter close to the eigenvalues sought. The transformation maps the eigenvalues $\Omega$ closer to $\sigma$ to the eigenvalues $\lambda$ with largest real part of the new matrix $(A-\sigma M)^{-1} M$, leaving the eigenvectors untouched.

To exploit the starsity pattern of the EVP, the implementation of the Arnoldi iterative method utlised for the recovery of the leading Ritz values and vectors has been done using the ARPACK software package. ARPACK is a collection of Fortran77 subroutines designed to solve large scale eigenvalue problems. This software is based upon an algorithmic variant of the Arnoldi process called the Implicitly Restarted Arnoldi Method (IRAM).

\section{Schematically, the EVP algorithm utilised is as follows:}

- - Only the non-zero elements of the discretised matrix $A$ and the diagonal matrix $M$ are stored as vectors using a sparse format.

- - Matrix $(A-\sigma M)$ is LU-decompsed.

- - Through an iterative procedure using ARPACK, approximations to the leading Ritz values and vectors of the linear operator $(A-\sigma M)^{-1} M$ are computed, the latter is post-processed in order to obtain the desired eigensystem of the original problem.

Second step in the scheme constitutes an improvement in the iterative Arnoldi procedure. ARPACK subroutines demand the repetitive solution of the linear system,

$$
(A-\sigma M) x=b,
$$

with identical left hand side, while the right hand side terms are updated in each iteration. A prior LU decomposition of the matrix $(A-\sigma M)$ will accelerate the linear system solver.

An LU-decomposition software with the capability to handle sparse data format is necessary. Recently, many new algorithms and software have emerged which 
exploit sparsity and new architectural features, such as memory hierarchu and parallelism. Several linear algebra packages for sparse matrices have been tested including Aztec, $S+$ and SuperLU. SuperLU is a general purpose library for the direct solution of large, sparse, non-symmetric systems of linear equations on high performance machines. The library is written in $\mathrm{C}$ and is callable form either $\mathrm{C}$ or Frotran. The library routines will perform and LU decomposition with partial pivoting and triangular system solves through forward and back substitution.

Regrettably, since the LU factorisation of a sparse matrix is not necessarily sparse, large matrices (as the one obtained in 3D problems) can not be allocated in memory, limiting the applicability of this method to 2D problems or small 3D problems.

Alternatives and those currently under investigation are Dynamic Mode Decomposition method $[26,94]$, which enter into the category of time-stepping method[22, 108], or instead of using LU factorisation to obtain the Krylov subspace, solve this system by iterative methods as Conjugate Gradients or GMRES.

\subsection{Adjoint global eigenvalue problem}

Adjoint formulation, introduced by Zhigulev[117], is related to the concepts of flow control and geometrical recptivity[10, 33, 45]. It can be used in the evaluation of the effects of initial conditions and forcing terms on the time evolution of the linearised Navier-Stokes equaitons (LNSE). Its utility in identifying flow receptivity and sensitivity is well recognised in both local[44, 90, 104], and global linear instability $[33,45]$. According to the difinition, the adjoint analysis gives the receptivity of the modes to the forcing in the momentum and/or the continuity equation.

We start from a simple eigenvalue problem, $A q=\omega q$, which can be written in the form

$$
(\omega+\Delta \omega)(q+\Delta q)=(A+\Delta A)(q+\Delta q)
$$

in which, $\Delta A$ is the given matrix perturbation and $\Delta \omega$ and $\Delta q$ are the resulting perturbation in eigenvalue and eigenvector, respectively. By rearranging the equation and multiplying by a vector $p$ gives:

$$
p^{H}(A-\omega I) \Delta q=p^{H}(\Delta A-\Delta \omega I) q,
$$

in which, the superscript ${ }^{H}$ indicates the complex conjugate transpose. Let the left hand side of the equation to be zero for all perturbations $\Delta q$, we get

$$
\begin{aligned}
& 0=p^{H}(A-\omega I), \\
& 0=\left(A^{H}-\omega^{*} I\right) p,
\end{aligned}
$$

in which, $p$ is the eigenvecotr of $A^{H}$, the adjoint matrix of $A$, also known as the called adjoint or left eigenvector of $A$. equation 3.23 indicates that the eigenvalues of the adjoint problem are the complex conjugates of the eigenvalues of $A$. 
As the inner product is defined

$$
<p, q>=p^{H} q
$$

we get

$$
<p, A q>=p^{H} A q=<A^{H} p, q>.
$$

With the weighted inner product,

$$
<p, q>=p^{H} M q
$$

which leads to

$$
<p, A q>=p^{H} M A q=p^{H} M A M^{-1} M q,
$$

where $M$ is the matrix of volumes, as in equation 3.4 and associated to the selected finite volume spatial discretisation. Then, the adjoint matrix is defined as:

$$
A_{\text {adj }}=M^{-1} A^{H} M \text {. }
$$

The adjoint modes can be obtained by solving an eigenvalue system, discussed in previous sections, as

$$
A_{a d j} q_{a d j}={ }_{a d j} M q_{a d j},
$$

in which, $\omega_{a d j}$ and $q_{a d j}$ are the eigenvalues and eigenvectors (here known as the adjoint eigenmodes), respectively.

Finally, from a computatinal point of, it is more efficient to define a vector $\overline{\bar{q}}_{a d j}=$ $M q_{a d j}$, such that the eigenvalue system equation 3.29 can be rearranged as:

$$
A^{H} \overline{\bar{q}}_{a d j}=\omega_{a d j} M \overline{\bar{q}}_{a d j} .
$$

Particularly, in the case where $A$ is a real matrix, $A^{H}=A^{T}$, To compare the last expression to the original direct eigenvalue system equation 3.4 , it is necessary to apply the Hermitian operator to equation 3.30, then we get:

$$
\overline{\bar{q}}_{a d j}^{H} A=\overline{\bar{\omega}}_{a d j}^{H} \overline{\bar{q}}_{a d j}^{H} M,
$$

in which it is assumed that, $B^{H}=B$, which is verified for the real diagonal matrix of cell volums. Equation 3.31 shows again, that the adjoint eigenvalues $\omega_{\text {adj }}$ are the compex conjugate eigenvalues of the original direct eigenvalue system and that the adjoint modes are the left eigenvectors of the original direct matris $A$.

\subsection{Structural sensitivity analysis}

The localised regions of sensitivity denote the spatial separation between direct and adjoint modes, which is related to the non-normality [16] of the Navier-Stokes equations. The regions where the direct and adjoint modes overlap define the structural sensitivity to localised feedback or wavemaker regions [32], and relates to the 
origin of absolute instabilities. These sensitivity maps provide information on the flow regions where a generic force-velocity coupling causes the largest drift in the eigenvalues and hence provides useful information on control strategies to attenuate these instabilities $[10,32,66]$. Other flow sensitivities, e.g. to base flow modification or steady forcing $[10,25,68,72,82]$, have been defined in the literature but are not considered here. Generally speaking, sensitivity analyses define the "sweet spots" for the location of passive control mechanisms and the regions where small modifications lead to the largest modification of the eigenmode behaviour (e.g. enhancing its stability).

The structural sensitivity maps can be calculated using the expression:

$$
S=\left\|q_{a d j}\right\| \cdot\|\hat{q}\|
$$

in which,

$$
<q_{a d j}, \hat{q}>=1,
$$

where and ${ }^{+}$are the direct and adjoint modes, where $<\bullet, \bullet>$ denotes the $L_{2}$ inner product, with its associated norm $\|\bullet\|=<\bullet, \bullet>^{1 / 2}$.

According to the theory of Giannetti and Luchini[33], the global feedback is modelled by a volume forcing in the momentum equations. Thus, just the velocity components of direct and adjoint modes, $\hat{q}$ and $q_{a d j}$ are used for the sensitivity computation. The localised region of structural sensitivity denotes the wide spatial separation between direct and adjoint modes, which is related to the non-normality of the compressible and turbulent NS equations[16]. Physically, the sensitivity region shows where flow modifications should be introduced in order to stabilise the system[55]. 



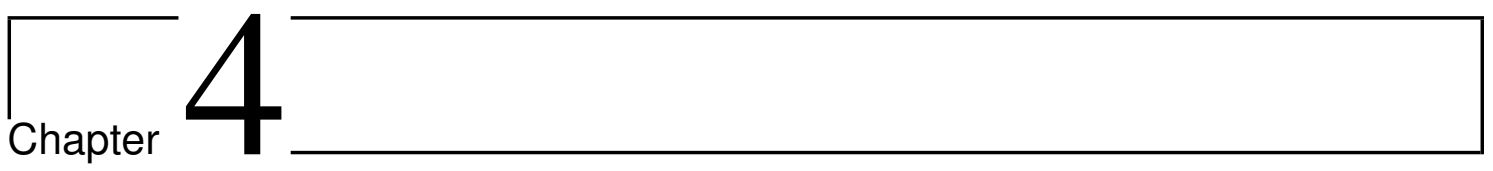

\section{Aerodynamic Optimisation}

\section{Contents}

4.1 Optimisation algorithm $\ldots \ldots \ldots \ldots \ldots \ldots \ldots$

4.2 Surrogate models $\ldots \ldots \ldots \ldots \ldots \ldots \ldots \ldots$

4.2.1 Radial basis funcion $(\mathrm{RBF}) \ldots \ldots \ldots \ldots \ldots$

4.2.2 Dynamically updated surrogate model . . . . . . . . . 38

4.3 Design of experiments . . . . . . . . . . . . 38

4.3.1 Full factorial design $\ldots \ldots \ldots \ldots$

4.3 .2 Orthogonal design . . . . . . . . . . . . . . 39

4.4 Parametric geometry representation . . . . . . . . . . 39

4.5 Mesh deformation: freeform deformation $\ldots \ldots \ldots \ldots 40$

4.6 Eigenmode tracking scheme . . . . . . . . . . . 41

4.7 Summary of aerodynamic optimisation based on stability analysis and sensitivity analysis . . . . . . . . . . 41

In this chapter, techniques of design optimisation envolved in aerodynamic optimisation are detailed. In particular, we introduce optimisation procedure based on genetic algorithm, geometric parametrisation with associated mesh deformation, an eigenmode tracking method that enables the identification of eigenmodes that are of our interest, surrogate modelling, and game theory for multi-objective optimisation.

\subsection{Optimisation algorithm}

The general reference to the optimisation problem in design is to select a set of parameters (variables) and to achieve the optimal design, subjected to a series of 


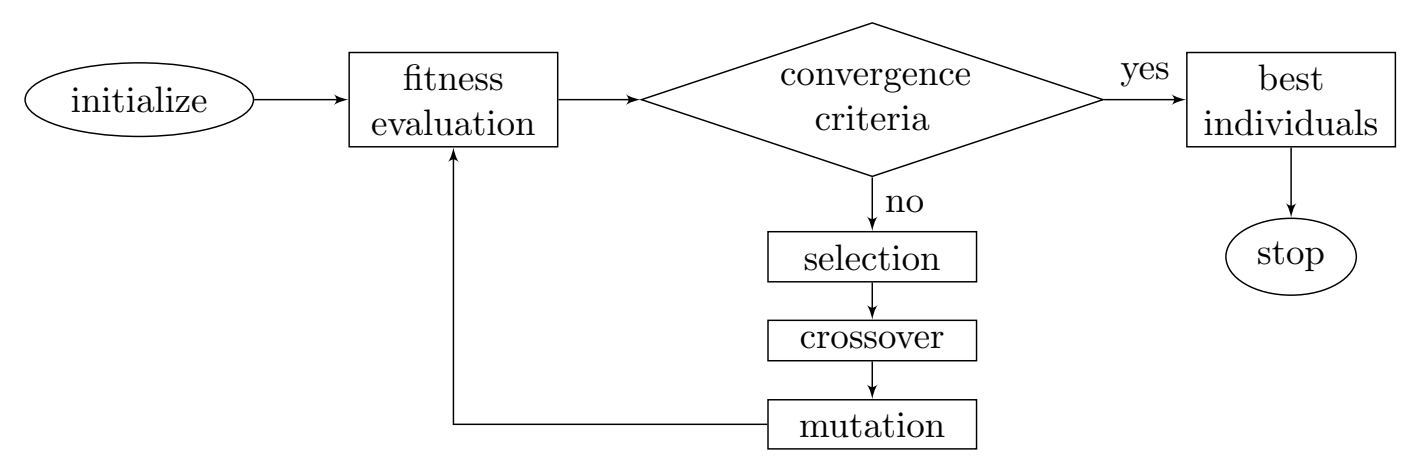

Figure 4.1: Flow chart of a simple genetic algorithm.

relevant constraints. Therefore, the optimization problem can usually be expressed as a mathematical planning problem. When carrying out optimisation design, the design problem should be expressed as a mathematical problem in the above form, and then solved by the optimisation method.

In recent decades, a number of optimisation algorithms have been introduced and deveoped, which are catagorised into derivative-free or derivative-based algorithms. Derivative-free optimisation algorithms mainly include genetic algorithm (GA), simulated annealing (SA), ant colony optimisation (ACO), etc. , while derivative-based optimisation is mainly consisted with gradient descent method, conjugated direction method, and conjugated gradient method, etc.. Considering the complexity and the scale of the problems considered in this thesis, GA is utilised in the investigations mentioned in this thesis.

Genetic algorithm was first introduced by John Holland in 1975[47], which emulates the process of natural selection and genetic mechanism in Darwin's theory of evolution, and seek the optimal solution through a natural genetic process [60]. The genetic algorithm starts with an initial population (a set of potential solutions), which consists of a number of individuals with combinations of different genes. A gene is a member in a parameter array of an individual. By calling a cost function, the fitness of each individual is evaluated, and the best individuals are selected. After crossover and mutation of genes, new generations with higher fitness are obtained. After a certain amount of iterations, an optimal solution is reached. The procedure of a typical genetic algorithm is shown in figure 4.1. In the algorithm, the number of maximum generations, the number of populations, the number of genes, the possibility of crossover and the possibility of mutations, need to be specified. The parameters retained for our optimisation are summarised in Table 5.6. Further details on genetic algorithms may be found in Ref. [17]. 
Table 4.1: Parameters selected for the Genetic Algorithm.

\begin{tabular}{cl} 
Name & Representation \\
\hline$N_{G}$ & Number of maximum generations \\
$N_{p}$ op & Number of population \\
$N_{g}$ & Number of genes \\
$P_{x}$ & Possibility of crossover \\
$P_{m}$ & Possibility of mutation
\end{tabular}

\subsection{Surrogate models}

Surrogate models are often adopted in the process of optimisation with complex cost functions, to reduce calls of cost function evaluation, reducing the impact of uncertainty in evaluation and for easier parallel off-line evaluation, which is particularly important when considering expensive evaluations such as when considering base flow and stability analysis. Surrogate models can be divided into two categories by whether they require gradient information or not. The ones without gradient information are more commonly utilised in global optimisation together with algorithms that do not require gradients of the cost functions, like genetic algorithm. There are a number of gradient-free surrogate models in the literature, including RSM (polynomial Response Surface Model), Kriging or RBFs (Radial Basis Functions) [84]. Among the surrogate models, the regression models like RSM are well suited for local optimisation problems with relatively simpler design space, while interpolation models such as Kriging and RBF can be used for highly-nonlinear multi-model functions, and thus well suited for global optimisation problems with complicated design space [38]. Alongside Kriging, RBF (Radial Basis Function) interpolation is a popular method for constructing surrogates suitable for highly non-linear data using irregular distributions of sample points, which can be adapted to the function being modelled. RBFs have seen increasing use for aerospace applications requiring an interpolation or approximation method, including mesh motion, fluid-structure interaction, and domain element parametrisation, in addition to aircraft data interpolation, owing to the meshless and general nature of the formulation, and tolerable properties of the interpolation kernel [67].

\subsubsection{Radial basis funcion (RBF)}

A Radial Basis Function (RBF) method is a linear combination of a series of basis functions, whose argument is the Euclidean distance between an interpolation point $\boldsymbol{p}$ and all the other points in the known data set. The model has the form:

$$
W(\boldsymbol{p})=\sum_{i=1}^{N} w_{i} \phi\left(\left\|\boldsymbol{p}-\boldsymbol{p}_{i}\right\|\right),
$$


where $W$ is the estimated value at location $p, \boldsymbol{p}$ is the interpolation point, $\phi$ is the chosen basis function, $w_{i}$ is the weight coefficient for each basis function, and $\|\bullet\|$ represents the Euclidean norm. Any function $\phi$ that satisfies the property $\phi(p)=\phi(\|p\|)$ is a radial function, and can be used as a basis function in RBFs. In this work, we select as many RBFs as the number of sampling points for the construction of the surrogate model (see next sections).

\subsubsection{Dynamically updated surrogate model}

Constructing a surrogate model with required presicion can be very expensive, especially when the cost functions are computationally expensive, which, in our case, takes both CFD simulation and stability analysis. As a trade-off of the precision of the surrogate model and computational cost, a surrogate model update scheme is introduced. After the optimisation algorithm i.e. GA finds an optimal, instead of stopping the optimisation procedure, we update the surrogate model with the actually value of the cost function (with CFD simulation and stability analysis), and restart the optimisation algorithm with the updated surrogate model. The flow chart of the construction of a surrogate model is shown in figure 4.2 . With this surrogate model update scheme, the sampling points get much denser near the optimal point, making the optimisation more precise.

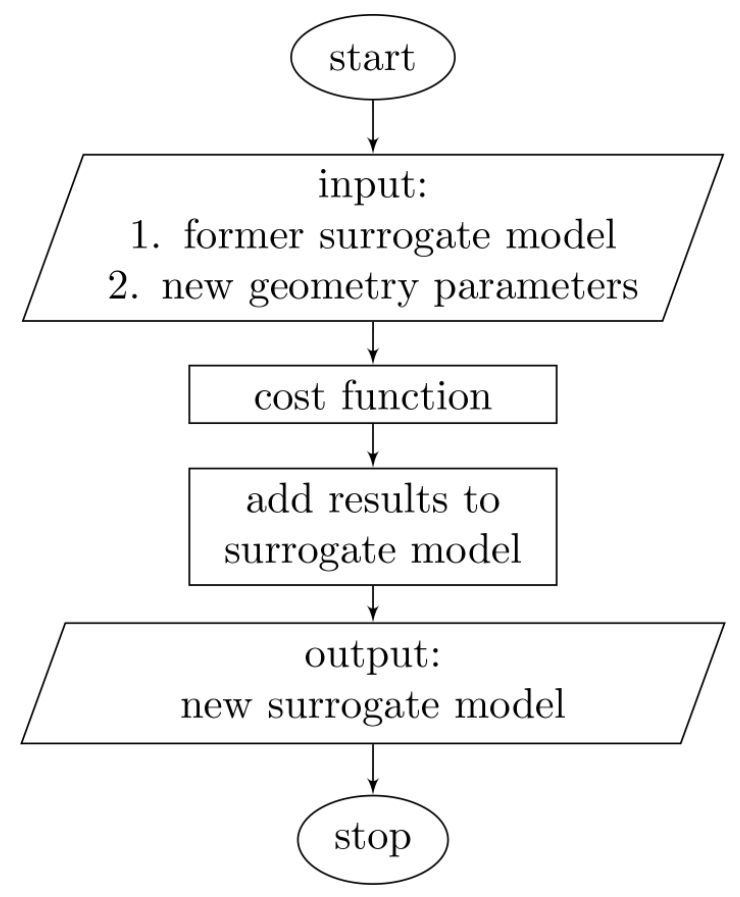

Figure 4.2: Construction of the surrogate model. 


\subsection{Design of experiments}

Design of Experiments enable building surrogate models with minimal sampling of the cost functions[116]. The most commonly employed experimental design methods are currently full factorial design, orthogonal design, uniform design, Latin hypercube sampling, central composite design, etc. Considering both the computational cost of the objective function evaluation and the construction of the efficiency and accuracy of the surrogate model, full factorial design and orthogonal design are adopted for difference optimisation problems in the present work.

\subsubsection{Full factorial design}

Full factorial design is the most conservative of all design types, of which, the sample size grows exponentially with the number of levels. In a full factorial design, we first devide the range of each design parameter into several sections (levels), and perform an experimental run at every combination fo the factor levels. The sample size is the product of the numbers of levels of the factors. Example are given in figure 4.3, shown two three-parameter full factorial designs, with three and four levels.

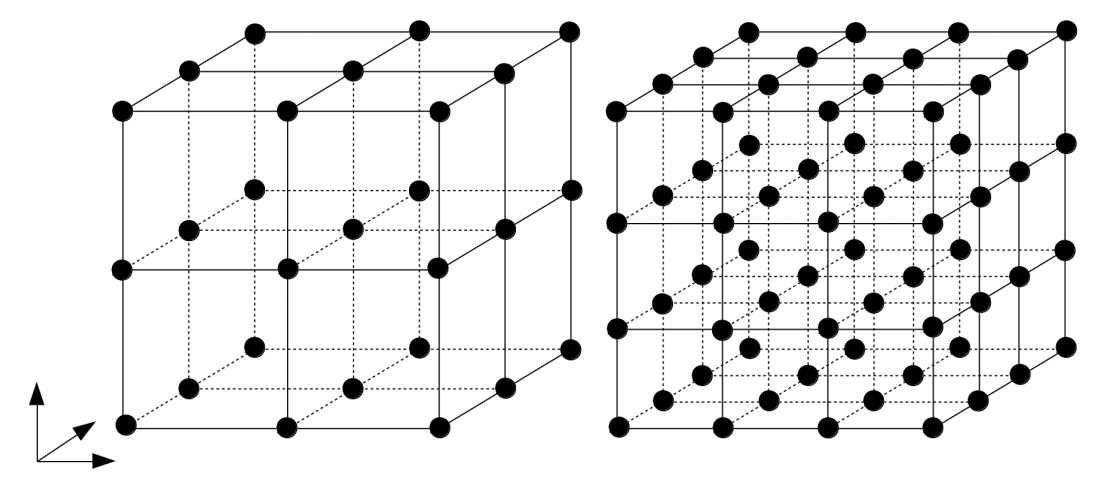

Figure 4.3: Three-parameter full factorial designs, with 3 and 4 levels.

\subsubsection{Orthogonal design}

Orthogonal experimental design, which is a partial design of full factorial design, is an efficient compromise between computational cost and accuracy of the surrogate model. In an orthogonal design, each level of each parameter is only considered once, as shown in figure 4.4 

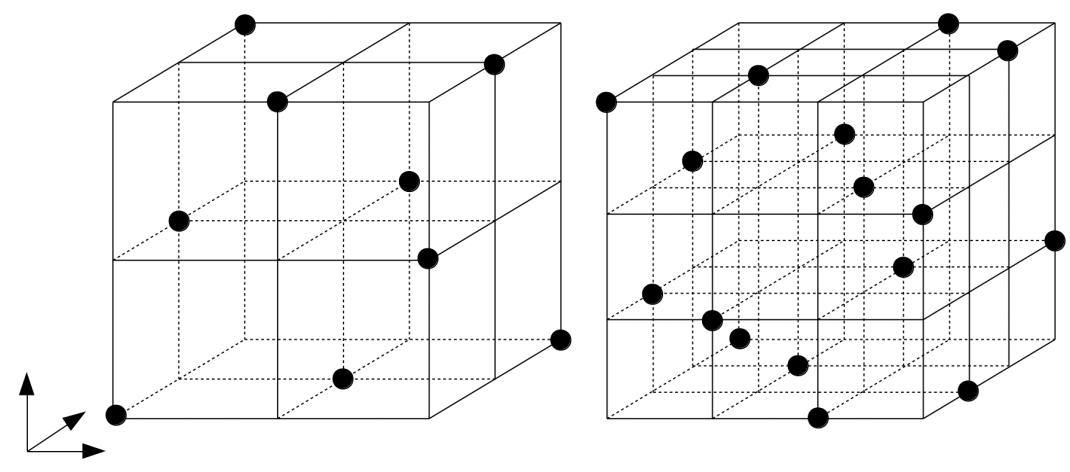

Figure 4.4: Examples of three-parameter orthogonal designs, with 3 and 4 levels.

\subsection{Parametric geometry representation}

Since different optimisation problems may involve very different geometry parametrisation, so it will be discussed along with different optimisation problems in the following chapters.

\subsection{Mesh deformation: freeform deformation}

In aerodynamic optimisation, the geometry shape will deform with the change of the optimisation parameters. To analyse with the new geometry shape, a new mesh is needed. There are two general ways to obtain a new mesh according to the new set of parameters. One is generating a new mesh from scratch with mesh generators, the other is to use mesh deformation techniques to get a new mesh based on a current mesh. Since an aerodynamic optimisation investigation requires a considerable number of sets of parameters, it is computationlly far less expensive to utilise a mesh deformation technique to get new meshes.

A simple FFD for 2-D mesh deformation was developed for investigations conducted within this thesis. The free form deformation acts on the boudnary mesh only, and the inner gird is deformed by this 2-D FFD program, in which, a bivariate $\mathrm{B}$-spline area is defined by:

$$
\vec{P}\left(u_{0}, v_{0}\right)=\sum_{i=0}^{n_{u}} \sum_{j=0}^{n_{v}} N_{i, m_{u}}\left(U_{0}\right) N_{j}, m_{v}\left(V_{0}\right) \vec{Q}_{i, j}
$$

with B-spline basis functions, calculated recursively:

$$
N_{i, m}(u)=\frac{u-u_{i}}{u_{i+m-1}-u_{i}} N_{i, m-1}(u)+\frac{u_{i+m}-u}{u_{i+m}-u_{i+1}} N_{i+1, m-1}(u)
$$


and,

$$
N_{u, 1}(u)= \begin{cases}1, & \text { if } u_{i} \leq u \leq u_{i+1} \\ 0, & \text { else }\end{cases}
$$

The control points $\vec{Q}_{i, j}$ define the basic deformation lattice, that encloses the object, defined by polygonal curves and surfaces, with points $\vec{P}_{\text {Object }}$. First all object points $\vec{P}_{\text {Object }}$ are mapped into the B-spline area by solving for $u_{0}, v_{0}$ using a Newton iteration method. Then the previously calculated $u_{0}, v_{0}$ are re-mapped to achieve the deformed objects points $\vec{P}_{\text {NewObject }}$ from:

$$
\vec{P}\left(u_{0}, v_{0}\right)=\sum_{i=0}^{n_{u}} \sum_{j=0}^{n_{v}} N_{i, m_{u}}\left(U_{0}\right) N_{j}, m_{v}\left(V_{0}\right) \vec{R}_{i, j}
$$

which has a different lattice control points $\vec{R}_{i, j}$, which determine the final deformed grid.

\subsection{Eigenmode tracking scheme}

To include the stability analysis in the optimisation loop, it is necessary to avoid manual identification of the eigenmodes obtained with new channel shape (i.e. identify the eigenmode responsible for the onset of asymmetry). A Modal Assurance Criterion (MAC) [59] is incorporated into the optimisation loop. The MAC method is an efficient way to track the modes by comparing sets of eigenmodes. To rank the similarity between eigenmodes, a MAC index is defined for a pair of eigenmodes $\hat{\mathbf{q}}_{i}$ and $\hat{\mathbf{q}}_{j}$ :

$$
\operatorname{MAC}\left(\hat{\boldsymbol{q}}_{i}, \hat{\boldsymbol{q}}_{j}\right)=\left(\frac{\left|\hat{\boldsymbol{q}}_{i} \cdot \hat{\boldsymbol{q}}_{j}\right|}{\left\|\hat{\boldsymbol{q}}_{i}\right\| \mid\left\|\hat{\boldsymbol{q}}_{j}\right\|}\right)^{2} .
$$

The MAC index approaches 1 when the pair of eigenmodes are linearly dependent (or identical), and is zero for orthogonal eigenmodes (not similar shapes).

The algorithm is more reliable when using eigenmodes computed on the same mesh. In this investigation, we modify the algorithm to allow comparison of eigenmodes in different meshes. The resulting method requires a few steps. First, all the the eigenmodes of interest are fed to the program. Then, the program process a first match to find the eigenmodes that have similar eigenvalues. After eliminating the ones with large eigenvalues differences, the program interpolates the eigenmode into the same mesh to generate a new set of comparable modes. Then, these are matched using the MAC method, which works well when using the same mesh. 


\subsection{Summary of aerodynamic optimisation based on stability analysis and sensitivity analysis}

In this section, the procedure of optimisation base on stability analysis and sensitivity analysis will be given.

First, based on the problem we are investigating, we conduct stability analysis to identify the mode that is causing the unfavourable phenomenon in the flow domain that we want to eleminate.

Second, we conduct sensitivity analysis to find the area in the flow field that affects this unwanted mode the most. It is at this region, we want to find an optimal set of parameters defining a passive or active flow control method to surpress this mode, making it stable, i.e. reducing the amplification rate of this mode to below zero.

Then, according to the complexity of the problem, we choose a design of experiment and construct the initial surrogate model, which is followed by an optimisation algrithm to find the optimal solution based on this surrogate model. In this procedure, the cost function, as shown in figure 4.5(a), will be evaluated several times, according to the design of experiment we choose.

Finally, we update the surrogate model by introducing the actual value of the cost function at the optimal point, and then restart the optimisation algorithm. We repeat this procedure until the error between the surrogate model at the optimal point and the actual value of the cost function is lower than a critical value. That is when we consider the optimisation has found an optimal design of the passive or active flow control.

The flowchart of the optimisation procedure is shown in figure 4.5(b), with $10^{-4}$ as the critical number of the error between the surrogate model at the optimal point and the actual value of the cost function is lower than a critical value. 


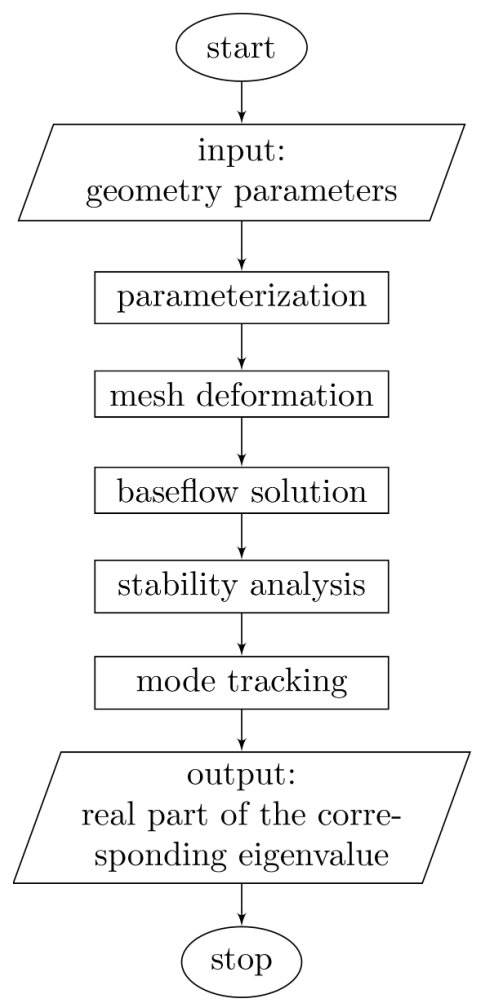

(a) Evaluation of the cost function.

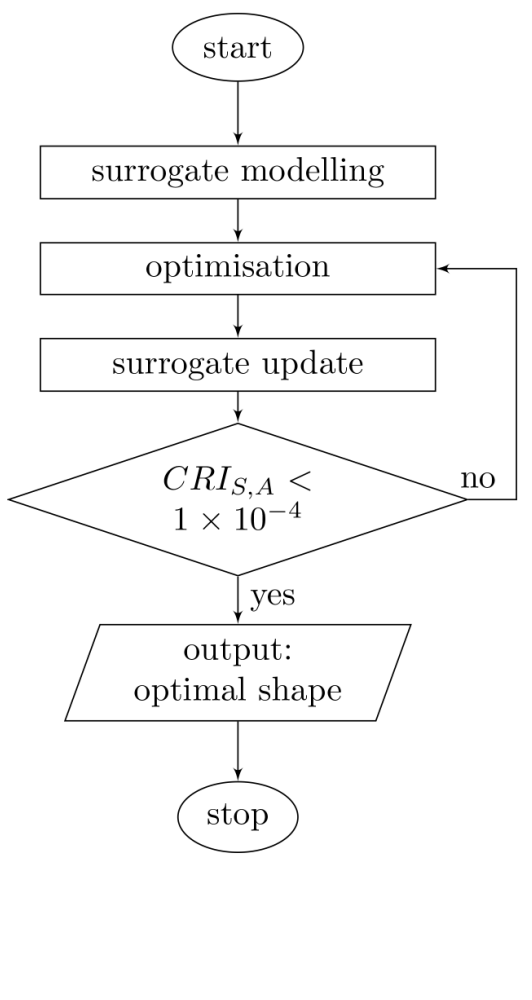

(b) Optimisation loop.

Figure 4.5: Optimisation flowchart. 



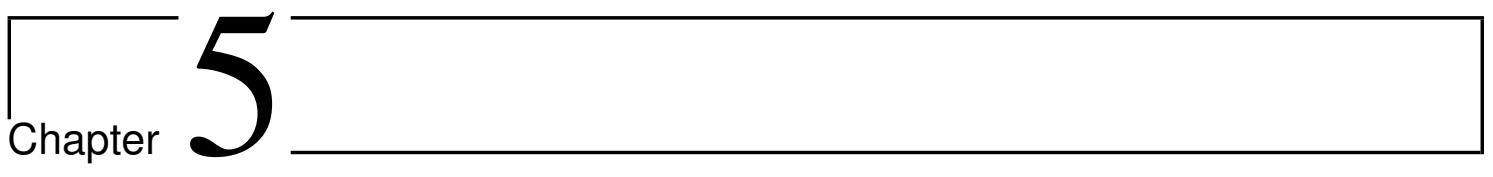

\section{Enhanced Stability of Flow through Contraction Channels}

\section{Contents}

5.1 Problem description . . . . . . . . . . . . . . . . 46

5.2 Base flow and onset of asymmetries . . . . . . . . . . 48

5.2 .1 Convergence study $\ldots \ldots \ldots \ldots \ldots \ldots$

5.2 .2 Numerical results . . . . . . . . . . . . . . . 50

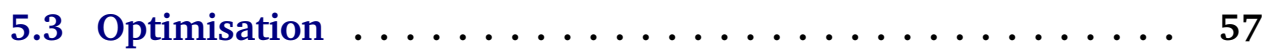

5.3 .1 Methodology . . . . . . . . . . . . . . . 58

5.3.2 Design of experiments . . . . . . . . . . . . . 60

5.3 .3 Optimisation results and discussion . . . . . . . . . . 61

5.3.4 The effect of optimisation on the drag reduction . . . . . . 67

5.3.5 Three-dimensional verification on the optimised configu-

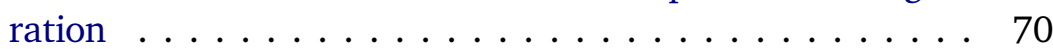

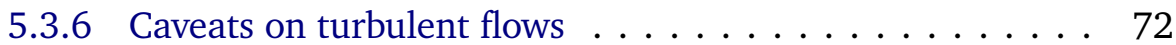

5.4 Summary and conclusions $\ldots \ldots \ldots \ldots \ldots \ldots \ldots \ldots$

In this chapter, we use the detailed methodology on a passive flow control technique, through shape optimisation, to enhance the stability of a contraction channel.

The first flow bifurcation, in channels with a sudden geometry contraction, is controlled through shape optimisation to delay the onset of asymmetry. First, we confirm the existence of a pitchfork type bifurcation instability, already reported in similar geometries. The global mode responsible for this bifurcation leads to asymmetric flow for Reynolds numbers beyond a critical value. Second, we propose a global shape optimisation methodology to introduce small modifications in 
the channel geometry that lead to flows with enhanced stability. Our results include three contraction ratios $C=2,4$ and 8 , where $C$ is the ratio of upstream to downstream channel widths.

The shape optimisation aims at minimising the growth rate of the unstable mode responsible for asymmetry. Sensitivity analysis is used to find an appropriate geometry parametrisation, which is defined through super-elliptic curves, and limited to small deformations. Additionally, a dynamically updated surrogate model (based on Radial Basis Functions) is developed to drive the optimisation. This substitutes expensive function evaluations, each requiring the solution of a steady Navier-Stokes base flow computation and a solution of an eigenvalue problem (linear stability analysis). Finally, a mode tracking algorithm identifies the eigenmode responsible for the onset of asymmetry during the optimisation.

The optimised geometries show rounded corners and are stable for Reynolds numbers well beyond the original values. For all contraction ratios, the critical Reynolds number increases by at least 7.9 times with respect to the original values. three-dimensional simulations confirm that the optimised geometry is more stable than the original when periodic boundaries are used on the side walls. When comparing the drag of the optimised geometry to the original, we obtain a reduction of at least $64 \%$.

\subsection{Problem description}

There have been several investigations aiming at understanding the flow behaviour in sudden contraction channels both numerically $[19,41,50]$ and experimentally [21]. In these geometries, two recirculation bubbles of different sizes are observed on each side downstream the contraction stage, once the critical Reynolds number is surpassed. Below the critical value, the flow remains symmetric. In the contraction channel, the Reynolds number $R e=D U / v$ is typically defined through the upstream channel height $D$, the mean inlet velocity $U$ and the kinematic viscosity $v$.

The experimental work of Cherdron et al.[13] and Sobey and Drazin[99], reported larger recirculation regions on one side of the channel. Sobey and Drazin proposed that the asymmetry could be associated to a shear-layer instability, which would be triggered for Reynolds numbers above the the critical value. These observations were numerically replicated by Chiang and Sheu[15], who showed that a supercritical pitchfork bifurcation (i.e. bifurcating from one fixed point into two fixed points [20]) is responsible for the loss of symmetry after the contraction. Once the symmetry is lost, a pressure gradient forms across the channel and leads to the well known Coanda effect [113].

Flow stability analysis [95] predicts how small flow perturbations grow or decay with respect to an equilibrium flow solution (the base flow), providing information about the onset of asymmetries or unsteadiness, the physical mechanism associ- 


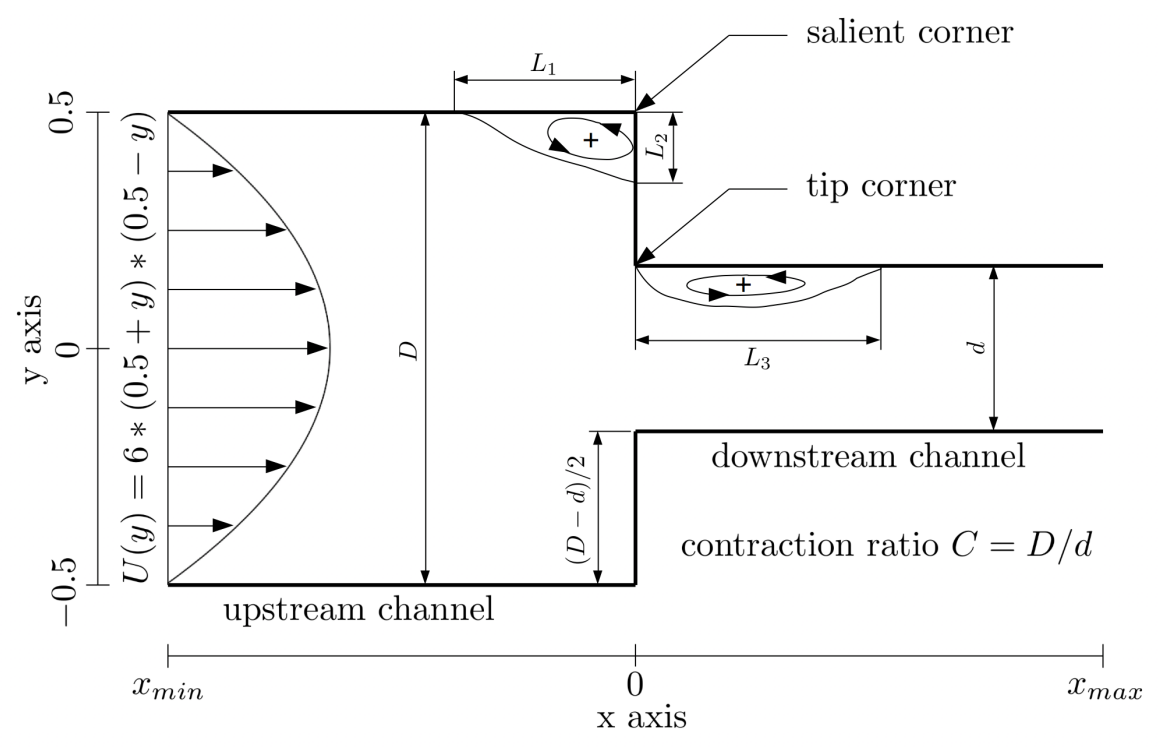

Figure 5.1: Geometry and the characteristic lengths.

ated to the perturbation responsible for the bifurcation, and can help to determine means by which to control it. This technique has been used to analyse similar geometries: sudden expansions [23, 24, 37, 97], sudden contractions [14, 15], geometries with both an expansion and a contraction [73], X-junctions [64], or more recently T-junctions $[12,61,106]$. The physical mechanism behind the onset of asymmetry, in these cases, is a stationary unstable global mode. Similar global instabilities have also been reported for flows behind bumps $[29,78]$, behind backward facing steps [6, 63, 69], in boundary layer recirculation bubbles [87] and in lid-driven cavities [1, 39].

The main objective of this work is to delay the onset of asymmetry (or increase the critical Reynolds number) in contraction channels. This task may be viewed as a passive flow control strategy, where asymmetry needs to be avoided for large values of the Reynolds number.

The geometry of contraction channel is depicted in figure 5.1. A parabolic horizontal velocity profile is imposed in the left inlet, with the form: $U(y)=$ $6 \times(0.5+y) \times(0.5-y)$. As shown in the figure, $D$ and $d$ are the widths of the channels upstream and downstream the contraction, respectively. Three contraction ratios will be subsequently considered: $C=D / d=2,4$ and 8 . Additionally, $\left(0-x_{\min }\right)$ and $\left(x_{\max }-0\right)$ are the lengths of upstream and downstream channels. The flow in this relatively simple geometry may be characterised by two separation regions $[13,15,99]$. The first at the upstream salient corner (before the contraction) and the second after the contraction and close to the tip corner. The second recirculation is sometimes referred to as separation bubble. Three geometric lengths define these two recirculation regions: $L_{1}$ is the separation length of the salient corner bubble, $L_{2}$ and $L_{3}$ are the reattachment lengths of the salient corner bubble 
Table 5.1: Geometry parameters and separation/reattachment lengths.

\begin{tabular}{clc} 
Name & Representation & Value \\
\hline$D$ & height of upstream channel & $1.00 \mathrm{~m}$ \\
$C$ & contraction ratio & $2,4,8$ \\
$d$ & height of downstream channel & $D / C$ \\
$x_{\max }-0$ & length of downstream channel & $5.00 \mathrm{~m}$ \\
$0-x_{\min }$ & length of upstream channel & $2.50 \mathrm{~m}$ \\
$L_{1}$ & separation length of salient corner bubble & \\
$L_{2}$ & reattachment length of salient corner bubble & \\
$L_{3}$ & reattachment length of tip corner bubble &
\end{tabular}

and the tip corner bubble respectively, which are defined as the distance between the separation point and the reattachment points on the walls.

A summary of the parameters describing the geometry and the flow in the contraction channel are shown in table 5.1. Finally, let us recall that the Reynolds number $R e=D U / v$ is typically defined, e.g. Ref. Chiang2002, through the upstream channel height $D$, the mean inlet velocity $U$ and the kinematic viscosity $v$.

\subsection{Base flow and onset of asymmetries}

\subsubsection{Convergence study}

We first include a grid convergence study for the steady base flows, based on the recirculation regions $\left(L_{1}, L_{2}\right.$ and $\left.L_{3}\right)$ defined in section 5.1. We select two Reynolds numbers $R e=1000$ and 2000 and a contraction ratio $C=2$, since these conditions enable comparisons with previously published results by Dennis and Smith[19], Hunt[50], Hawken et al.[41] and Chiang and Sheu[15]. Results are summarised in table 5.2. We consider that grid independence is achieved, when the results of two successive grids are smaller than 5 percent, for the $L_{1}, L_{2}$ and $L_{3}$ lengths. The biggest difference in lengths between the last two sets of grids is 2 percent, so the results we obtain from the grid $h=1 / 1280(2560 \times 1280$ elements $)$ can be considered independent of the mesh. This last mesh has 2 million elements, which leads to very costly computations. Instead of using homogeneous mesh refinement, it is more efficient to use a comparatively coarse mesh with local refinements, where mesh points are clustered near the channel corners. The results from the locally refined mesh are shown in comparison with uniform meshes in table 5.2. By using the locally refined mesh, the computation time is reduced to only 5 percent of that of the finest uniform mesh, while the accuracy is comparable. The final parameters used for the locally refined mesh are included in table 5.3. Additionally, let us note that the converged results show good agreement with previous published data. 
Table 5.2: Grid convergence: Separation and reattachment lengths $L_{1}, L_{2}$ and $L_{3}$ for contraction ratio $C=2$.

\begin{tabular}{lcccccc}
\multicolumn{1}{c}{ Length } & \multicolumn{2}{c}{$L_{1}$} & \multicolumn{2}{c}{$L_{2}$} & \multicolumn{2}{c}{$L_{3}$} \\
\hline Reynolds number & 1000 & 2000 & 1000 & 2000 & 1000 & 2000 \\
\hline Dennis and Smith[19] & 0.138 & 0.182 & 0.081 & 0.093 & & \\
Hunt[50] & 0.154 & 0.197 & 0.82 & 0.094 & 0.207 & 0.481 \\
Hauken et al.[41] & 0.143 & & 0.077 & & 0.239 & \\
Chiang and Sheu[15] & 0.140 & 0.183 & 0.082 & 0.094 & 0.222 & 0.543 \\
Present: $160 \times 80, \quad \mathrm{~h}=1 / 80$ & 0 & 0.062 & 0 & 0.006 & 0 & 0 \\
$320 \times 160, \quad \mathrm{~h}=1 / 160$ & 0.031 & 0.031 & 0.013 & 0.018 & 0.060 & 0.241 \\
$640 \times 320, \quad \mathrm{~h}=1 / 320$ & 0.143 & 0.185 & 0.063 & 0.068 & 0.182 & 0.453 \\
$1280 \times 640, \quad \mathrm{~h}=1 / 640$ & 0.143 & 0.187 & 0.079 & 0.089 & 0.239 & 0.548 \\
$2560 \times 1280, \quad \mathrm{~h}=1 / 1280$ & 0.144 & 0.193 & 0.081 & 0.092 & 0.242 & 0.550 \\
$\quad$ locally refined & 0.143 & 0.189 & 0.081 & 0.090 & 0.241 & 0.550
\end{tabular}

Table 5.3: Detailed parameters of the locally refined mesh.

$$
\begin{array}{ccccc}
C & N_{\Delta x} & N_{\Delta y} & \left(\Delta x_{\min }, \Delta x_{\max }\right) & \left(\Delta y_{\min }, \Delta y_{\max }\right) \\
\hline 2,4,8 & 320 & 360 & (0.001,0.012) & (0.0005,0.003)
\end{array}
$$

Having checked the convergence of the base flows, we now proceed to inspect the convergence of the eigenvalues. To this aim, we compare the eigenvalues for the asymmetric mode at $R e=2500$ and 4000 for $C=2$, in table 5.4. We select higher Reynolds ( $R e=2500$ and 4000) since these represent a symmetric (stable) and an asymmetric (unstable) state for the contraction ratio $C=2$, respectively. As for the base flow, convergence is obtained for the grid $h=1 / 1280(2560 \times 1280$ elements). The eigenvalues obtained for the locally refined mesh are identical (with the retained accuracy of $10^{-3}$ ) to the finest homogeneous mesh.

Finally, retaining the locally refined mesh, we analyse the influence of the Krylov

Table 5.4: Grid convergence fro eigenvalues: real part $\left(\sigma_{R}\right)$ of the most unstable asymmetric mode, for contraction ratio $C=2$.

\begin{tabular}{lccc}
\multicolumn{2}{l}{ Reynolds number } & 2500 & 4000 \\
\hline uniform: & $40 \times 20$ & -0.193 & -0.142 \\
$80 \times 40$ & -0.241 & -0.173 \\
$160 \times 80$ & -0.197 & -0.094 \\
$320 \times 160$ & -0.205 & 1.378 \\
$640 \times 320$ & -0.253 & 1.623 \\
$1280 \times 640$ & -0.294 & 1.763 \\
$2560 \times 1280$ & -0.294 & 1.764 \\
locally refined & -0.294 & 1.764
\end{tabular}


Table 5.5: Krylov subspace dimensions convergence for eigenvalues: Real part $\left(\sigma_{R}\right)$ of the most unstable asymmetric mode, for contraction ratio $C=2$.

\begin{tabular}{clcc}
\multicolumn{2}{c}{ Reynolds number } & 2500 & 4000 \\
\hline Krylov & 40 & -0.293 & 1.437 \\
dimension: & 80 & -0.294 & 1.734 \\
& 160 & -0.294 & 1.764 \\
& 320 & -0.294 & 1.764
\end{tabular}

space size in the convergence of the eigenvalues, when using the Arnoldi method. The Krylov subspace dimension controls the accuracy of the eigenvalues for the Arnoldi methods. table 5.5 shows that a Krylov space if dimension 160 is sufficient to obtain accurate eigenvalues for the two Reynolds numbers. Finally, note that to obtain the previous eigenvalue convergence for grid refinement, table 5.4, we selected 320 to avoid Krylov dimensions effects.

In summary, the results included in subsequent sections use local refinement (see table 5.3) and Krylov spaces of size 320.

\subsubsection{Numerical results}

We select the recirculation length $L_{3}$ as the criterion to determine when flow symmetry is lost and the flow bifurcates following a supercritical pitchfork bifurcation, see figure 5.2. We depict the $L_{3}$ bubble size against Reynolds numbers for contraction ratios $C=2,4$ and 8 , in figure 5.2. Note that due to the symmetry of the channel, the large bubble can appear near the upper or the lower corner randomly and consequently, we choose the same symbol to show the sizes of bubbles when considering the full domain simulations in figure 5.2. Additionally, when the Reynolds number is below the critical value, the two curves overlap. The critical Reynolds number where the flow bifurcates and asymmetries appear decreases for increasing contraction ratios. We obtain critical Reynolds: $R e_{c r i}=3150$ for $C=2$, $R e_{c r i}=1420$ for $C=4$ and $R e_{c r i}=1230$ for $C=8$. Similar bifurcation diagrams and critical Reynolds numbers were reported by Chiang and Sheu[15].

Additionally, figure 5.2 shows that the reattachment lengths of the two recirculation bubbles (at both sides of the contraction) become larger as the Reynolds number increases. Lashgari et al.[64] suggested that the point of the bifurcation is determined by the size of the recirculation region, in a X-junction flow. However, in our contraction channel, the size of the recirculation region at the critical Reynolds number shows a nonlinear decrease for increasing contraction ratios. This may be seen in figure 5.2, where the approximate bubble sizes are 0.8 for $C=2,0.5$ for $C=4$ and 0.4 for $C=8$. In both configurations nonetheless, the mechanism responsible for bifurcation is comparable. As the recirculation bubbles grow in size, the contraction becomes effectively smaller, such that the side bubbles approach, which facilitates the vertical momentum transfer. This mechanism will be further 


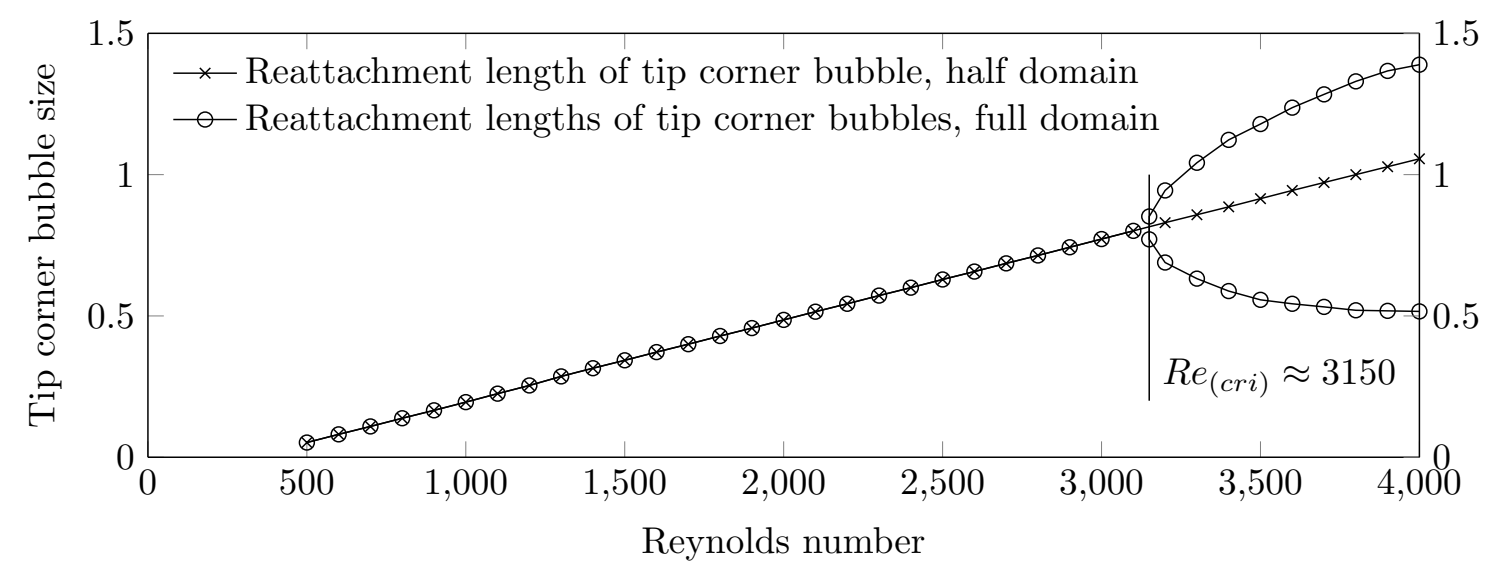

(a) $C=2$.

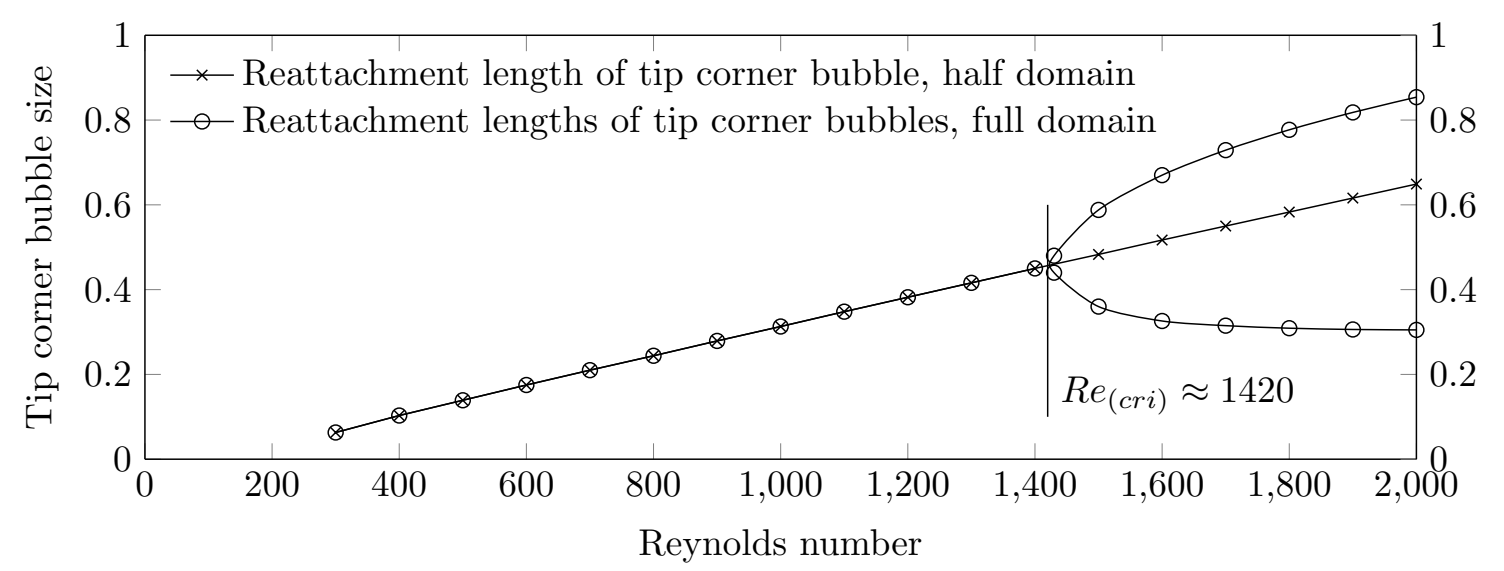

(b) $C=4$.

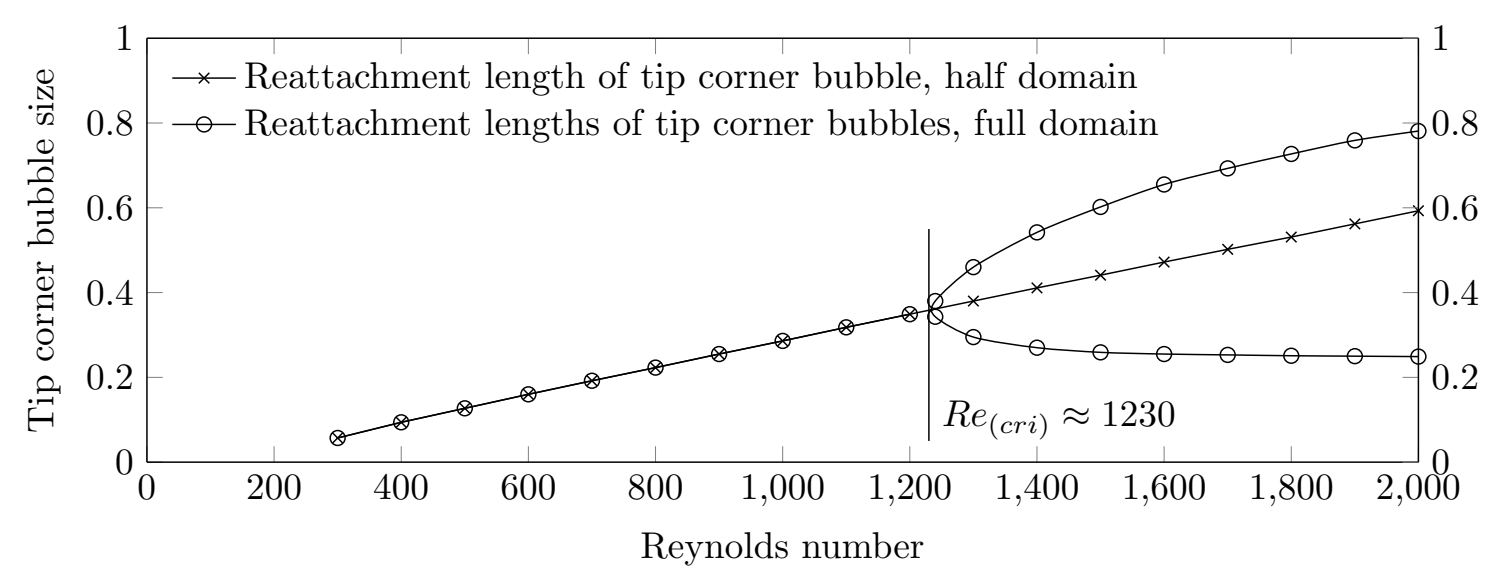

(c) $C=8$.

Figure 5.2: Bifurcation diagram for contraction ratios $C=2,4$ and 8, illustrated using the reattachment lengths $L_{3}$ of downstream tip corner eddy for varying Reynolds numbers. 
explored in following sections and controlled through the proposed shape optimisation.

We illustrate the symmetric and asymmetric flows in figure 5.3, where we present results for Reynolds numbers 1000 and 2000 for contraction ratio $C=4$. For Reynolds number 1000, the simulation converges to a symmetric flow, see Figs. 5.3(a) and 5.3(c). Two recirculation bubbles of the same size form downstream of the contraction and near the tip corners. Further downstream, due to the viscous dissipation, the flow regains its Poiseuille channel flow profile. When the Reynolds number is increased to 2000, an asymmetric steady flow develops, see Figs. 5.3(b) and 5.3(d). In the asymmetric case, two recirculation bubbles of unequal size develop and lead to a lost of symmetry and the well reported Coanda effect [113]. Figs. 5.3(c) and 5.3(d) detail the recirculation regions. The Coanda effect is a consequence of the pressure difference established in the cross-stream direction [99]. Note that the new asymmetric flow is steady and no periodic flow develops, confirming that the bifurcation is of pitchfork type.

[40] suggested that asymmetries resulted from the interaction between the destabilising upstream convection effects by the asymmetric perturbation and the combined stabilising effects of the viscous dissipation and the downstream convection of perturbations by the base symmetric flow. However, Chiang and Sheu[15] and Lashgari[64] have shown that an absolute instability is responsible for the pitchfork bifurcation resulting in lost of symmetry. This will be confirmed in the next section through linear stability analyses.

Finally and for completeness, we depict in figure 5.4 the recirculation regions for various Reynolds numbers. It can be seen that the bubble becomes larger when the Reynolds number increases. Additionally, we observe that for asymmetric unstable flows, unequal bubbles develop.

\subsubsection{Stability analysis}

To better understand the nature of the presented bifurcations, we conduct linear stability analyses for Reynolds number ranges $R e=2500$ to $4000, R e=900$ to 2000 and $R e=900$ to 2000, for contraction ratios $C=2,4$ and 8, respectively. In figure 5.5 , we present the evolution of the growth rates (or real part of the eigenvalue) of the least stable or unstable eigenvalue, against the Reynolds number. We include the critical Reynolds number at which the eigenvalue crosses the real axis, indicating the onset of instability. By comparing these results to the previous bifurcation diagrams (see figure 5.2) in section 5.2.2, it is clear that the bifurcations start at exactly the same Reynolds numbers, which indicates that the selected eigenvalues govern the onset of asymmetry.

Additionally, Figs. 5.6(a) and 5.6(b) illustrate the eigenvalues for contraction ratio $C=4$ and Reynolds numbers $R e=1000$ and 2000, and mark the least stable asymmetric mode with a filled square. We observe that for $R e=1000$ all eigenvalues have negative real part, which indicates that the flow is stable, whilst for 


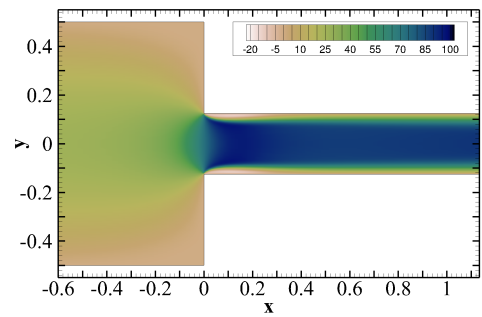

(a) $R e=1000$.

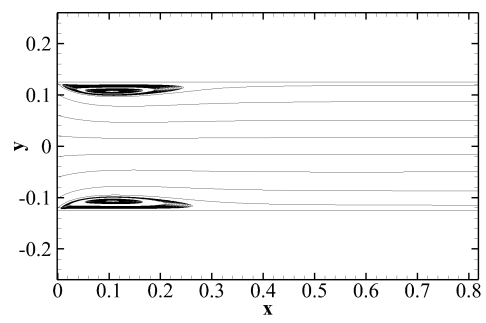

(c) $R e=1000$, zoomed region.

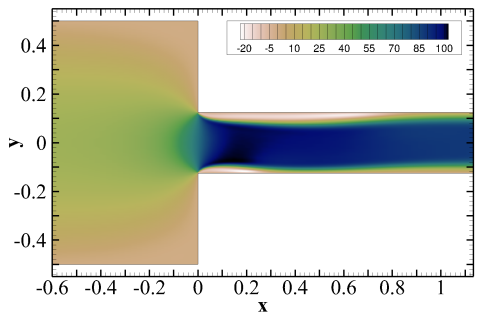

(b) $R e=2000$.

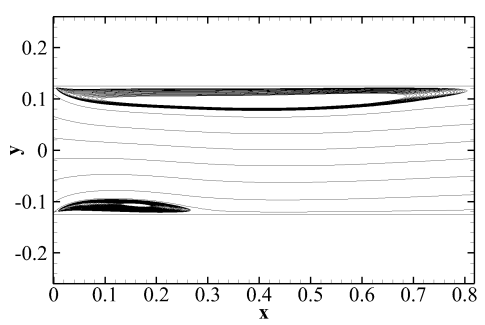

(d) $R e=2000$, zoomed region.

Figure 5.3: Horizontal velocity component $U$ (a,b) and streamlines (c,d) for $R e=$ 1000 and $R e=2000$, showing symmetric and asymmetric recirculation bubbles, respectively. Contraction ratio is $C=4$ in all cases.

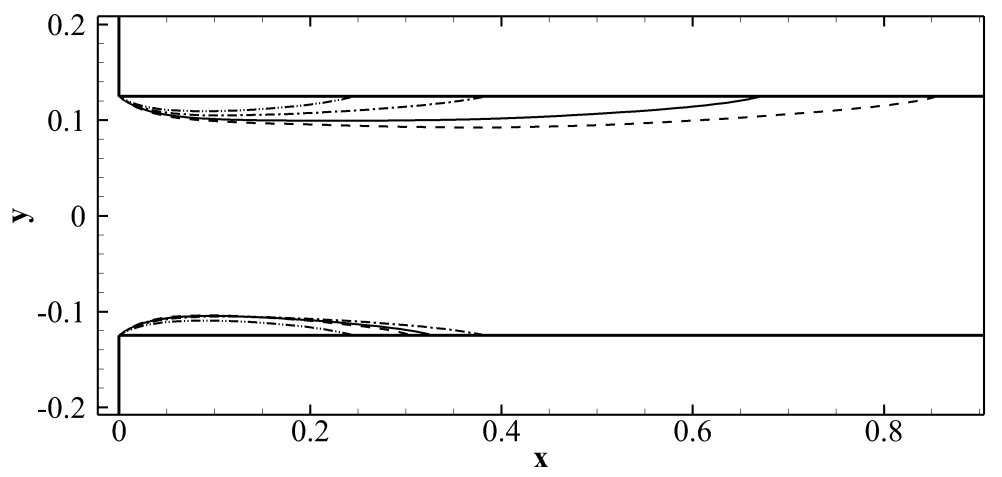

Figure 5.4: Recirculation bubbles for contraction ratio $C=4$ at $R e=800$ (dash dot dot), $R e=1200$ (dash dot), $R e=1600$ (solid) and $R e=2000$ (dash). 


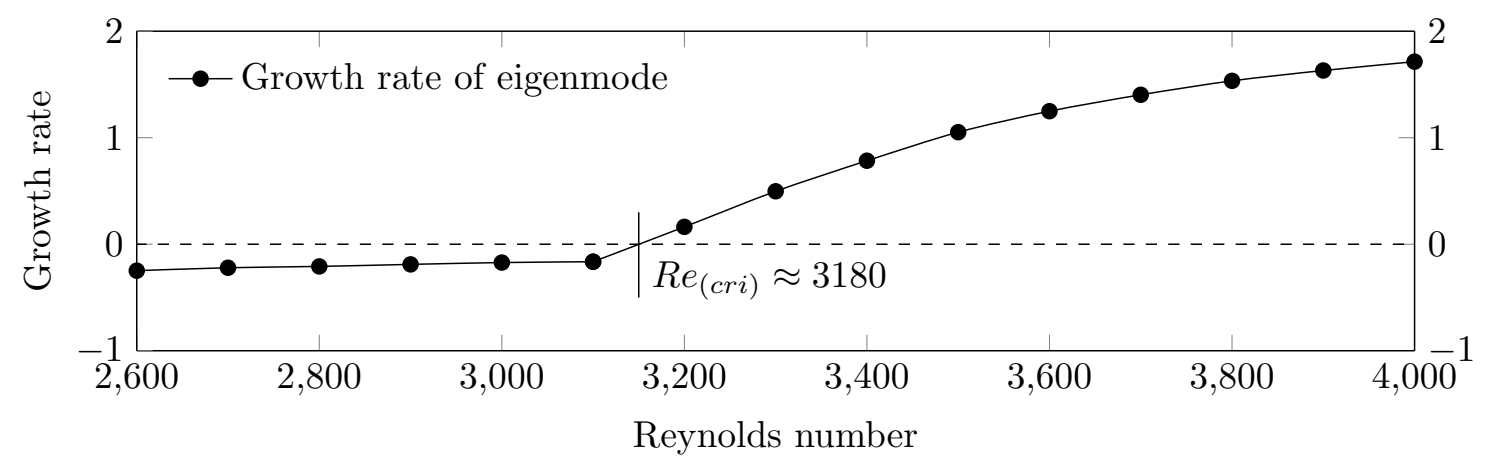

(a) $C=2$.

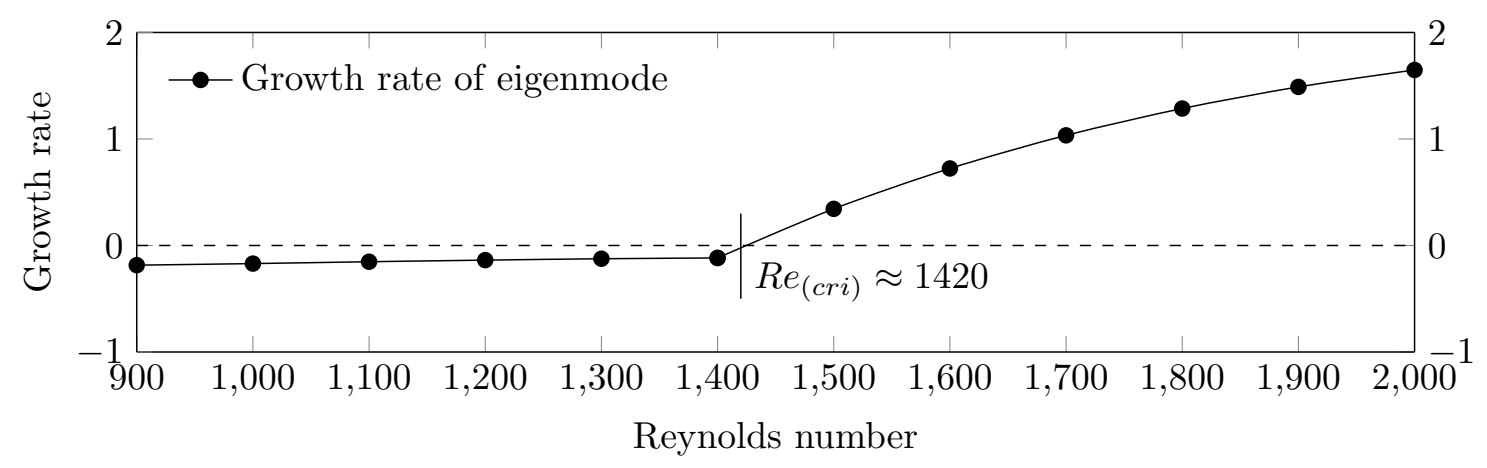

(b) $C=4$.

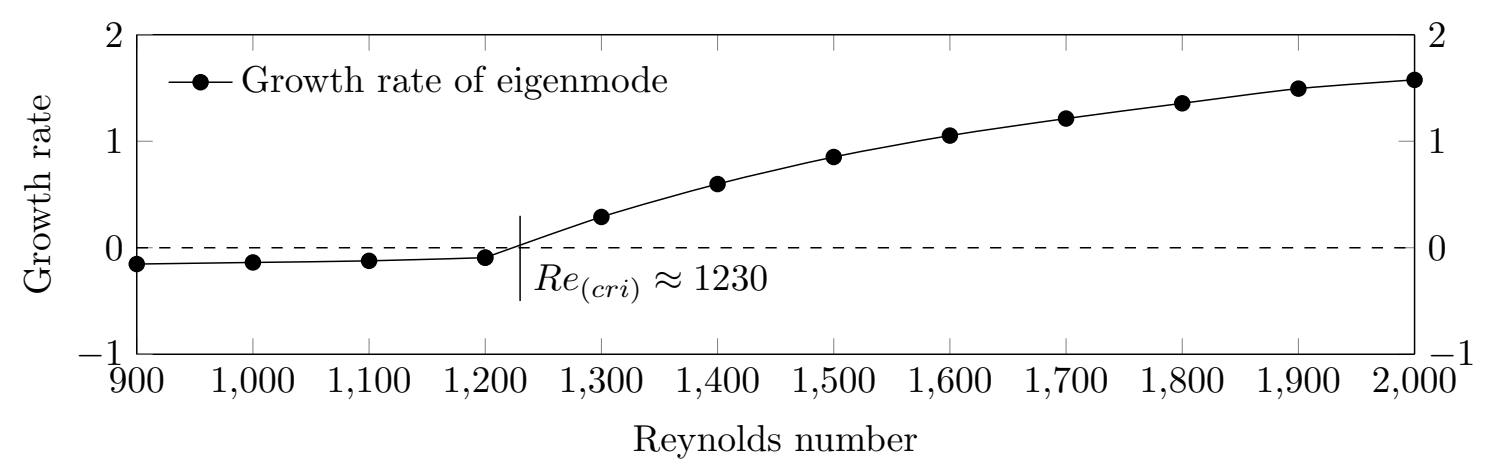

(c) $C=8$.

Figure 5.5: Growth rates (real part of eigenvalues) against Reynolds numbers, for $C=2,4$ and 8 . 
$R e=2000$, there is one unstable mode (positive real part), which is obtained by analysing the symmetric unstable flow obtained through mirroring half domain. Note that all modes are complex but the modes without imaginary component (e.g. asymmetric modes) represent flow structures without temporal oscillating frequencies.

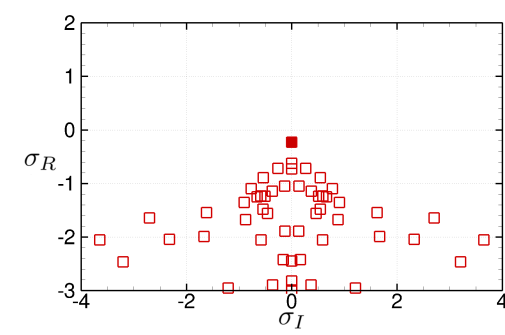

(a) $\operatorname{Re}=1000$.

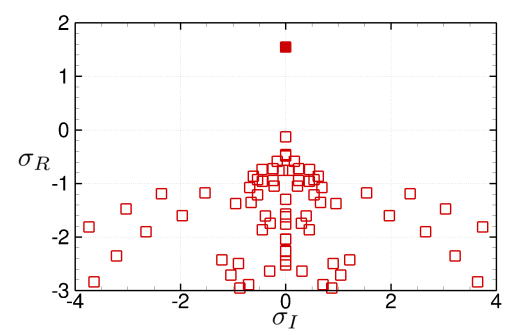

(b) $R e=2000$.

Figure 5.6: Eigenvalue spectra for $R e=1000$ and 2000 for contraction ratio $C=4$. The least stable mode is marked with a filled square.

The eigenmodes corresponding to the marked eigenvalues (filled squares) are depicted in figure 5.6. Particularly, we depict the component of the eigenmode corresponding to the streamwise velocity in Figs. 5.7(a) and 5.7(b).We observe that for both Reynolds numbers, the eigenmodes are asymmetric. These modes are responsible for the first bifurcation and the loss of symmetry. The $u$ perturbation, shown in Figs. 5.7(a) and 5.7(b), is responsible for the increased speed at the outer edge of the top bubble and the decrease at the outer edge of the bottom bubble. The vertical velocity component perturbation $(v)$ (not shown) modifies the flow upwards at $x=0.2$ and downwards at $x=0.7$. The structure of these global modes shows that the streamwise acceleration leads to lower pressure at the upper recirculation area, and higher pressure at the lower recirculation area, which in turn induces a cross-stream pressure gradient that helps to maintain the asymmetric flow profile.

In addition, Figs. 5.7(c) and 5.7(d) show the adjoint modes corresponding to the previous direct modes. It is noticeable that the adjoint modes are located near the salient corner and the tip corner, denoting the importance of these corners in the receptivity of the direct modes and the stability of the flow.

\subsubsection{Structural sensitivity}

The regions with highest structural sensitivity indicate the zones where a localised modification will lead to significant changes in the mode and will need to be considered to control the flow. This sensitivity map is obtained by overlapping the direct 


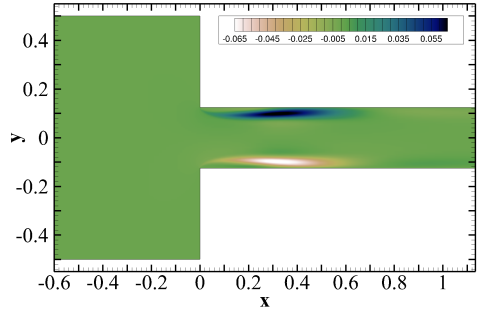

(a) Direct eigenmode, $R e=1000$.

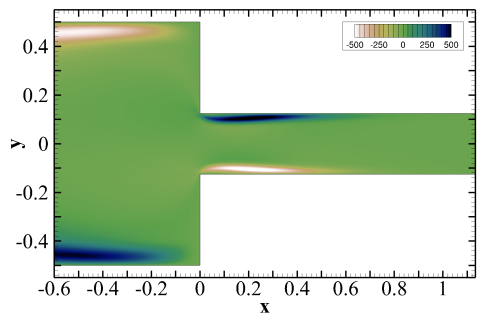

(c) Adjoint eigenmode, $R e=1000$.

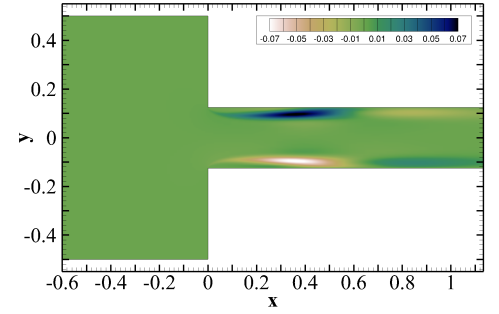

(b) Direct eigenmode, $R e=2000$.

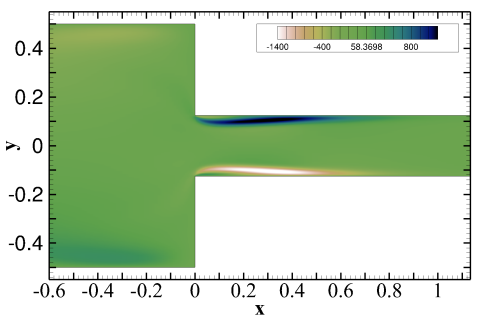

(d) Adjoint eigenmode, $R e=2000$.

Figure 5.7: Direct (a,b) and adjoint (c,d) eigenmodes for $R e=1000$ and $R e=2000$, for contraction ratio $C=4$. All show horizontal velocity component $u$.

and adjoint global modes, as shown in figure 5.8, where the recirculation line (in red) has also been included for reference.

The highest structural sensitivity appears near the edge of the recirculation bubbles and extends from the contraction corner. This shape suggests that by manipulating the geometry of the corner and the surrounding region, the instability may be controlled.

The spatial map of the production of the perturbation kinetic energy is shown in figure 5.8(b) for the same flow, and has been normalised by the overall perturbation kinetic energy in the domain. We found that the region with largest transfer of the kinetic energy from the base state to the perturbations are located in the shear layer at the edge of the recirculation bubbles, and the core of the instability is found to be near the region with maximum positive and negative production. More specifically, we compute the contribution of the lift-up perturbation kinetic energy term: $u v d U / d y$, shown in figure 5.8(c). Comparing Figs. 5.8(b) and 5.8(c), we can see that the energy production by the term $u v d U / d y$ and the total energy production are almost identical in shape and amplitude, confirming that a lift-up mechanism is responsible for the onset of asymmetry. Similar conclusions have been reported by [64] for an X-junction geometry. The lift-up mechanism, where the disturbance extracts energy from the base flow through the term $u v d U / d y$, is common in several shear flow instabilities such as Kelvin-Helmholtz instabilities 


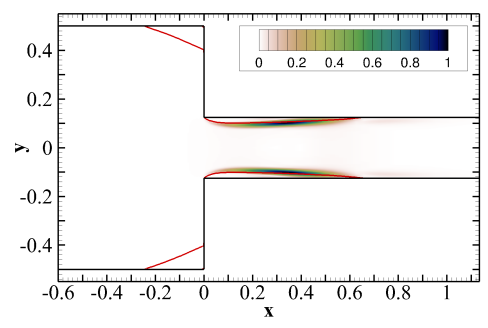

(a) Spatial map of structural sensitivity.

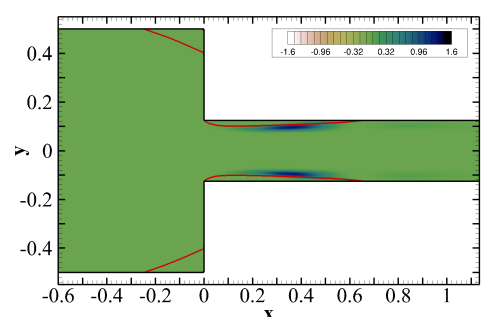

(b) Production of the perturbation energy.

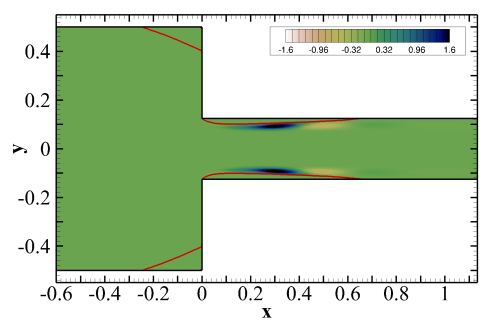

(c) Lift-up perturbation: $u v d U / d y$.

Figure 5.8: Structural sensitivity map, Production of kinetic energy and lift-up kinetic energy perturbation, at $R e=2000$ for contraction ratio $C=4$. Red lines indicate the edge of recirculation regions.

or Tollmien-Schlichting waves. Further details may be found elsewhere [9].

In summary, we have reproduced published results to verify that the onset of asymmetry is governed by an absolute instability (an asymmetric mode). When computing the adjoint of this mode and associated structural sensitivity, we observe a region of high sensitivity near the contraction, starting at the corner and extending downstream. In what follows, we will parametrise the geometry to enable a shape optimisation that can modify the geometry of the sharp corners (by a small amount), to enhance the stability of the problem.

\subsection{Optimisation}

In this section, we first detail the methodology for shape optimisation used in this investigation. In particular, we introduce the optimisation procedure based on a genetic algorithm, the parametrisation of the geometry (and associated mesh deformation), the mode tracking method that enables the identification of the most unstable asymmetric eigenmodes and the surrogate model enabling an efficient optimisation. 
Table 5.6: Parameters selected for the Genetic Algorithm.

\begin{tabular}{clc} 
Name & Representation & Value \\
\hline$N_{G}$ & Number of maximum generations & 500 \\
$N_{p}$ op & Number of population & 100 \\
$N_{g}$ & Number of genes & 3 \\
$P_{x}$ & Possibility of crossover & 0.6 \\
$P_{m}$ & Possibility of mutation & 0.6
\end{tabular}

\subsubsection{Methodology}

Bearing in mind that real part of the eigenvalue $\left(\sigma_{R}\right)$ stands for the growth rate of eigenmodes, the optimisation includes the minimisation of a cost function, which is defined through the real part of the eigenvalue responsible for the onset if asymmetry $\left.\sigma_{R}\right|_{\text {assym. }}$. This eigenvalue is a function of the parameters $\boldsymbol{p}$ defining the channel geometry. Based on this assumption, the optimisation problem may be stated as:

$$
\begin{aligned}
& \left.\min \sigma_{R}\right|_{\text {assym }}(\boldsymbol{p}) \\
& \text { subject to }: \quad h_{i}(\boldsymbol{p})=0, \text { for } i=1,2
\end{aligned}
$$

in which, $\boldsymbol{p}$ is the set of selected parameters defining the geometry (defined in the next section). The flows in the new optimised shapes need to fulfil at all times the Navier-Stokes equation (2.1) and the eigenvalue problem equation (3.4), both defined in previous sections. More specifically, these constraints may be stated by imposing $h_{1}=\mathscr{M} \frac{\partial \boldsymbol{q}}{\partial t}-\mathscr{F}(\boldsymbol{q})$ and $h_{2}=\mathscr{J}(\overline{\boldsymbol{q}}) \hat{\boldsymbol{q}}-\sigma \mathscr{M} \hat{\boldsymbol{q}}$ in the minimisation problem equation (5.1).

Based on the optimisation problem, the optimisation loop requires a surrogate model, a geometry parametrisation, a mesh deformation, a base flow simulation, a stability analysis and a mode tracking. The main loop is started by building the initial surrogate model. Then, followed by the loop of optimisation and surrogate model updating.

The parameters retained for our optimisation are summarised in table 5.6. Further details on genetic algorithms may be found in Ref. 1.davis1991handbook-ofgen.

\subsubsection{Parametrisation and mesh deformation}

The geometry is parametrised using super-elliptic curves of the form:

$$
\left|\frac{x}{a}\right|^{1 / p}+\left|\frac{y}{b}\right|^{1 / p}=1
$$

In our implementation, we set $a=b=1$, as it proves favourable that the major and minor axis of the super-ellipse are equal, to reduce the number of the geometry parameters and computational cost. With higher degree $p$, the curvature radius 
Table 5.7: Range for the geometry parameters (or design space): $\boldsymbol{p}=\left(p_{1}, p_{2}, p_{3}\right)$.

\begin{tabular}{cccc} 
Parameter & Lower bound & Upper bound & Original \\
\hline$p_{1}$ & 0.0 & 1.0 & 0.0 \\
$p_{2}$ & 0.0 & 1.0 & 0.0 \\
$p_{3}$ & $-2.5^{\circ}$ & $2.5^{\circ}$ & 0.0
\end{tabular}

becomes smaller and enables the definition of a straight angle as $p$ tends to zero or infinity, as shown in the figure 5.9. This parametrisation provides a continuous curvature at the intersection point between the parametrised part and the remaining geometry, allowing smooth transitions between different boundary sections. This last property helps to deform the computational mesh and alleviates the apparition of distorted elements.

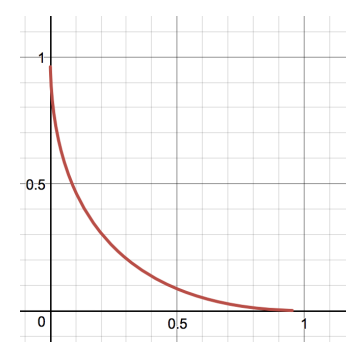

(a) $(1.0,1.0,0.5)$.

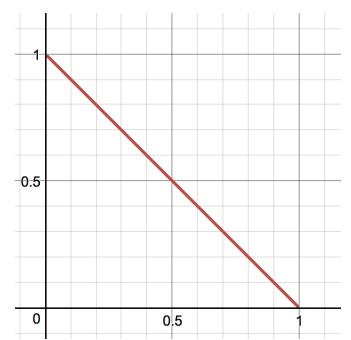

(b) $(1.0,1.0,1.0)$.

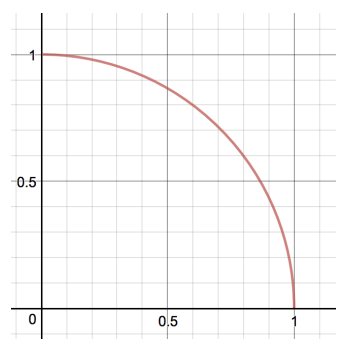

(c) $(1.0,1.0,2.0)$.

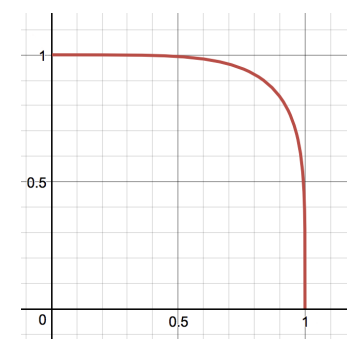

(d) $(1.0,1.0,5.0)$.

Figure 5.9: Super-elliptic curves with different degrees, with parameters $(a, b, 1 / p)$.

As shown in figure 5.7 and discussed in section 5.2.2.1, the mode responsible for the onset of asymmetries is located near the salient corner and the tip corner. Consequently, we parametrise the geometry such that the optimisation algorithm can reshape the geometry near these regions. The top corner and salient corner (at the contraction stage) are parametrised as two distinct super-elliptic curves, with exponents in equation (5.2) being $p_{1}$ for the top and $p_{2}$ for the salient corner. As shown in figure 5.9, the degree of the super-elliptic curve controls the curvature of the corners. An additional parameter is included to control the tangential direction of the contraction stage with respect to the vertical direction. In summary, the set of parameters for optimisation $\boldsymbol{p}=\left(p_{1}, p_{2}, p_{3}\right)$, see equation (5.1), include a parametrisation of the two corners through $p_{1}, p_{2}$. Additionally, $p_{3}$ governs the angle (in degrees) of the contraction stage with respect to vertical direction. These parameters are sketched in figure 5.10 and their ranges (or complete design space) are summarised in table 5.7. Let us note that the selected range of parameters only allow small deformations, whilst retaining the overall shape of the contraction channel.

For completeness, we show examples of the parametrisation in figure 5.11, the 


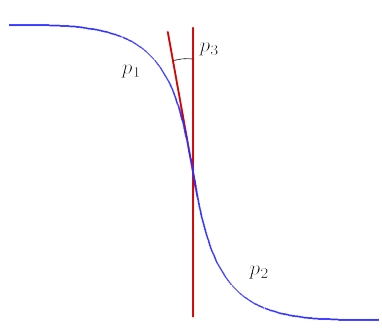

Figure 5.10: Geometry parametrisation using the parameters $\boldsymbol{p}=\left(p_{1}, p_{2}, p_{3}\right)$ at the contraction stage.

the minimum and maximum values for each parameter and the resulting shapes are illustrated.

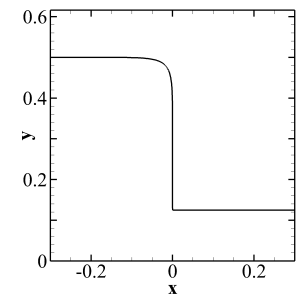

(a) $\left(1.0,0.0,0.0^{\circ}\right)$.

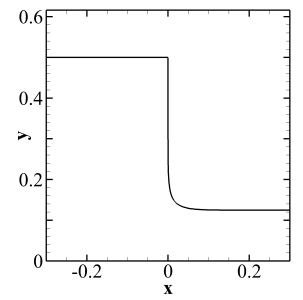

(b) $\left(0.0,1.0,0.0^{\circ}\right)$.

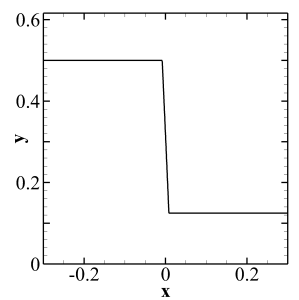

(c) $\left(0.0,0.0,2.5^{\circ}\right)$.

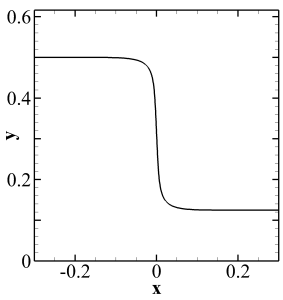

(d) $\left(1.0,1.0,2.5^{\circ}\right)$.

Figure 5.11: Examples of geometries using the geometry parametrisation $\boldsymbol{p}=$ $\left(p_{1}, p_{2}, p_{3}\right)$.

Since the shape optimisation will deform the geometry, it is important to control the mesh deformation to avoid unusable meshes (e.g. with negative or distorted elements). To control the mesh deformation, a Free-form deformation (FFD) method is developed based on Ref. Jamshid2004. The FFD acts on the two-dimensional boundary mesh deforming the inner grid, whilst maintaining the quality of the mesh. Some examples of the mesh deformation are given with different geometry parameters in figure 5.12, where it can be seen that the mesh is fine enough even in the modified geometries.

\subsubsection{Design of experiments}

For the current investigation, as a trade-off of computational cost of the precision of surrogate model, instead of having $L^{N}$ sampling points in a full factorial design, an orthogonal design has $L^{N-1}$ sampling points, having $L$ levels for each parameter. In this work, there are $N=3$ parameters $\left(p_{1}, p_{2}, p_{3}\right)$, each of which takes $L=5$ different levels (or degrees) making a $L^{N-1}=5^{2}$ orthogonal design experiment, with 25 sampling points. 


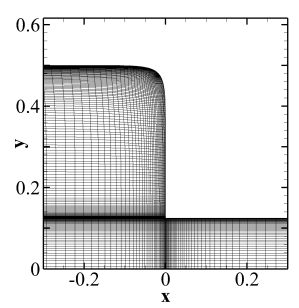

(a)

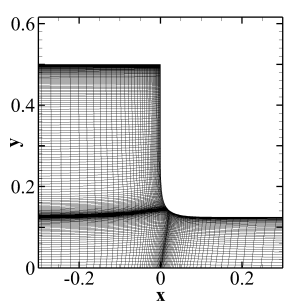

(b)

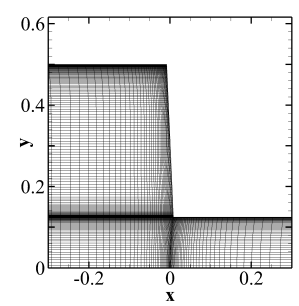

(c)

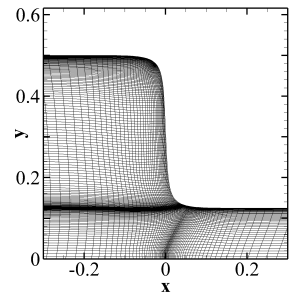

(d)

Figure 5.12: Examples of mesh deformation for the geometries depicted in figure 5.11 .

\subsubsection{Optimisation results and discussion}

In this section, we discuss the result obtained through shape optimisation of the contraction channel, with the aim of increasing the critical Reynolds number. To obtain results that may be compared to optimisations based on stability and sensitivity analyses (e.g. Lashgari et al. [64] for sensitivities to blowing/suction), we only allow small shape deformations. We select one unstable Reynolds number for each contraction ratio $\left(R e_{o p t}\right.$, slightly higher that the critical Reynolds number, see figure 5.2), and optimise. We choose $R e_{o p t}=3200$ for $C=2, R e_{o p t}=1500$ for $C=4$ and $R e_{\text {opt }}=1300$ for $C=8$.

In this optimisation investigation, we select two criteria to check the convergence of the optimisation procedure, $C R I_{S, A}$ and $C r i_{G A}$. First, in the procedure of surrogate model (shown in figure 4.2), we check the relative change of the cost function value obtained from the surrogate model of the current generation compared to the last generation, $\mathrm{Cri}_{G A}$. Second, in the optimisation loop (shown in figure ??), we monitor the error of the optimal obtained by GA from the surrogate and compare it to the value of cost function from the actual simulation at this given point, $C R I_{S, A}$. We choose $C R I_{S, A}<1 \times 10^{-4}$ and $C r i_{G A}<1 \times 10^{-3}$ as the convergence criteria for the present optimisation investigation.

In Figs. 5.13, we show the convergence process of the optimisation on the contraction channels with three contraction ratios. It is noticeable that the error of the surrogate model is un-neglectable in the beginning. However, with the dynamical updating scheme, the error decreases as the optimisation progresses. In less than 20 iterations, the error of the surrogate model, which is the criterion we choose for the optimisation $\left(C R I_{S, A}\right)$, becomes lower than $1 \times 10^{-4}$, and the optimisation is terminated. It is also shown that the grow rates of the eigenmodes causing the bifurcation are greatly reduced to below zero. Total number of iterations taken to converge $\left(N_{c}\right)$ and computing hours $\left(T_{c}\right)$ are shown in table 5.8 for the three contraction ratios.

When the optimal shape is obtained, it is important to test the optimal shape 


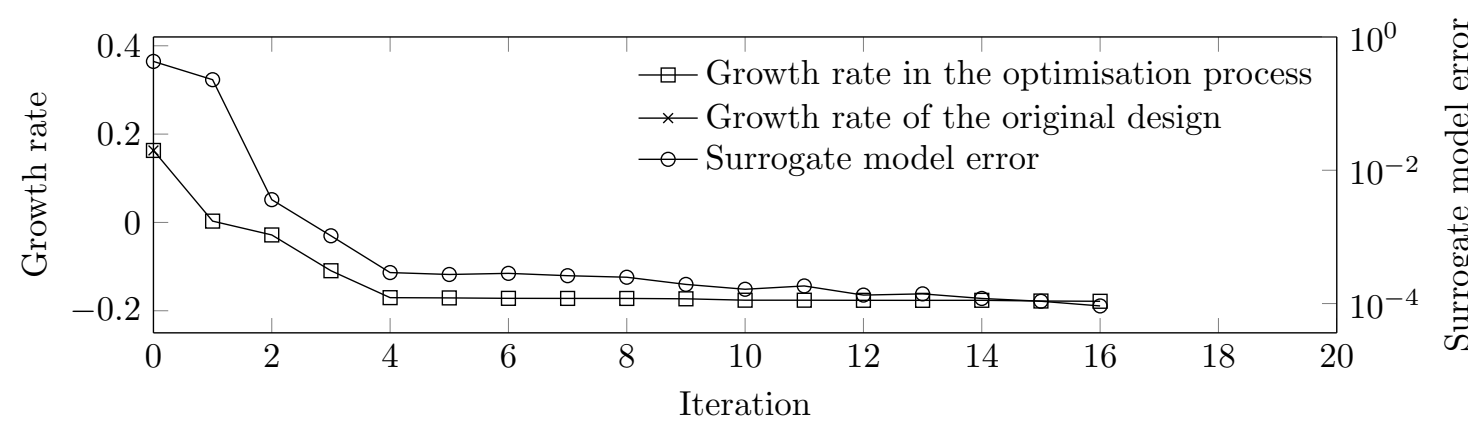

0
0
0
0
0
0
0
0
0
0
0
0
0
0
0
0

(a) $C=2$.

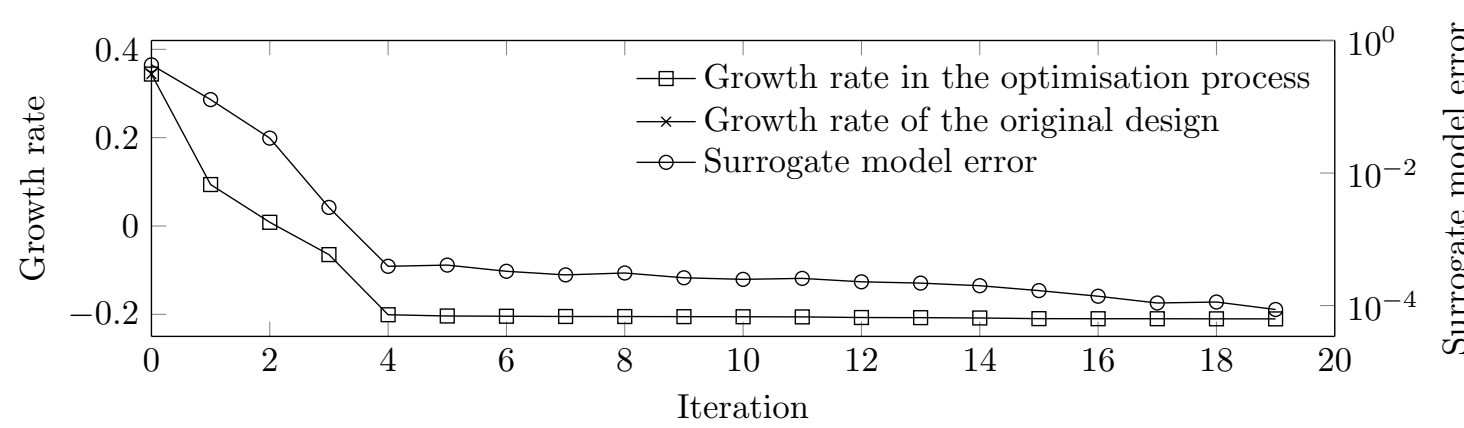

(b) $C=4$.

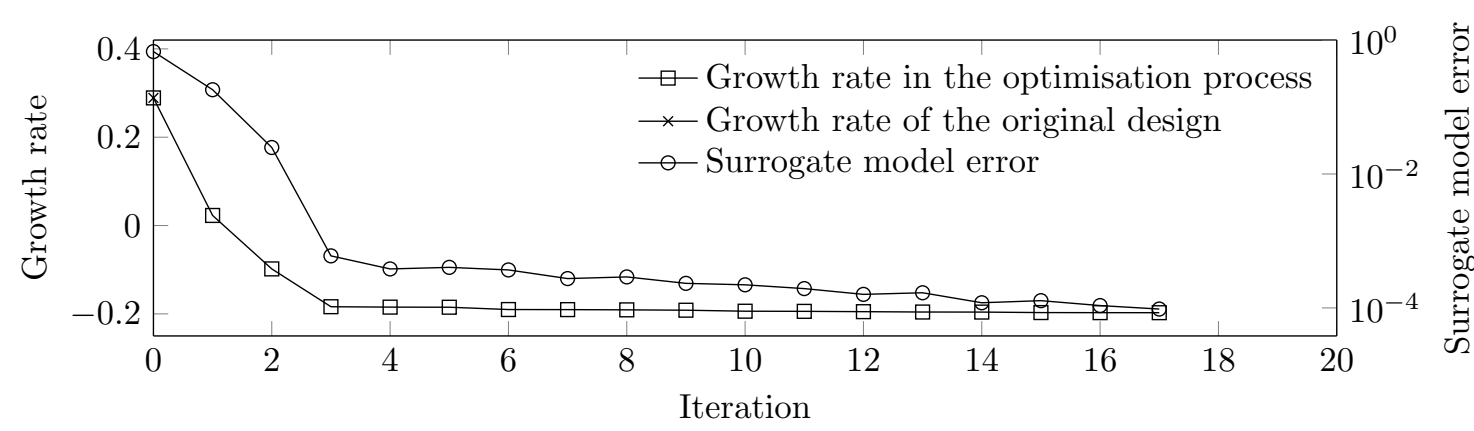

(c) $C=8$.

Figure 5.13: Convergence process of the optimisation.

Table 5.8: Total number of iterations and computational time taken for the optimisation.

\begin{tabular}{ccc}
$C$ & $N_{c}$ & $T_{c}$ (core $\times$ hour $)$ \\
\hline 2 & 16 & 337 \\
4 & 19 & 427 \\
8 & 17 & 439
\end{tabular}


against some random suboptimal shapes, as previous study by Tammisola [103]. After the optimisation is converged, we compare the optimised geometries to the geometries consisting the sample group of the surrogate model and the geometries updated to the surrogate model. All these geometries show less stable flow comparing to the optimal shape obtained after the optimisation is converged.

The shapes resulting from the optimisation are depicted in Figs. 5.14, 5.15 and 5.16. In these figures, the original shapes have also been included for comparison. In all cases, we observe that the tip and salient corners have been rounded and that the contraction stages (vertical walls) have been tilted.

We can see in the eigenvalue spectra, shown in Figs. 5.14(b), 5.15(b) and 5.16(b) in comparison with Figs. 5.14(a), 5.15(a) and 5.16(a) that the growth rates of the most unstable asymmetric modes for each contraction ratio are reduced and below zero in the optimised shapes. It is clear that the optimisation has stabilised the mode responsible for asymmetries but also that the shape deformation has not destabilised other modes. The corresponding eigenmodes are shown in Figs. 5.14(d), 5.15(d) and 5.16(d) in comparison with Figs. 5.14(c), 5.15(c) and 5.16(c). The modes in the optimised geometries are thinner and have been relocated from behind the tip corners to around the corner. These new modes lie along the boundaries following the the curved corner. It is noticeable how small changes in the geometry modify substantially the eigenmodes.

Additionally, we show the base flows for the original and optimised geometry configurations. Results for the optimised shapes are shown in Figs. 5.14(f), 5.15(f) and 5.16(f), in comparison with the original results shown in Figs. 5.14(e), 5.15(e) and 5.16(e). After the optimisation, we observe a significant reduction of the velocity gradients at the contraction. Furthermore, the flows are all symmetric and the sizes of the recirculation bubbles are either greatly reduced $(C=2)$ or even completely eliminated ( $C=4$ and $C=8$ ).

Sensitivity analyses are compared for the original and optimised configurations (and for the three contraction ratios). The structural sensitivity maps for the optimised configurations are shown in Figs. 5.14(h), 5.15(h) and 5.16(h), which can be compared to the sensitivities of the original configurations, Figs. 5.14(g), $5.15(\mathrm{~g})$ and $5.16(\mathrm{~g})$. For all three contraction ratios, the regions with high sensitivity have been relocated from near the tip corner bubbles to the salient corner bubbles, which shows the beneficial effect of the optimisation in stabilising the mode responsible for asymmetries. Additionally, the lift-up perturbation kinetic energy ( $u v d U / d y$ ) has almost disappeared illustrating how the new shapes minimise the lift-up mechanisms resulting in more stable configurations. It is important to note that structural sensitivity and lift-up term show that the physical mechanism behind the pitch fork bifurcation has been changed by very small modification in the geometry.

We now analyse the sensitivity of the shape parameters selected for optimisation with respect to the growth rate. figure 5.17 shows the influence of the parameters in the growth rates of the asymmetric modes, and provides valuable information 


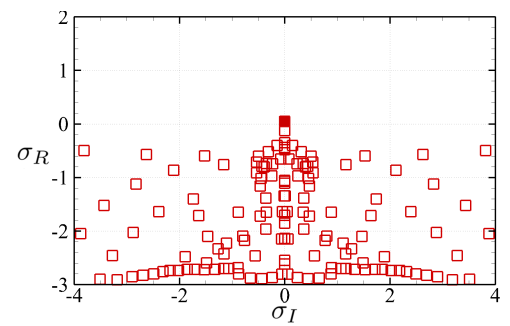

(a) Original eigenvalue spectrum.

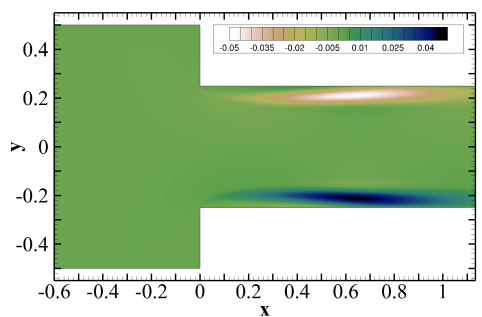

(c) Original eigenmode (horizontal velocity).

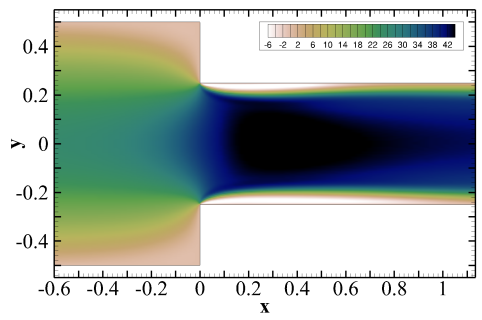

(e) Original base flow (horizontal velocity).

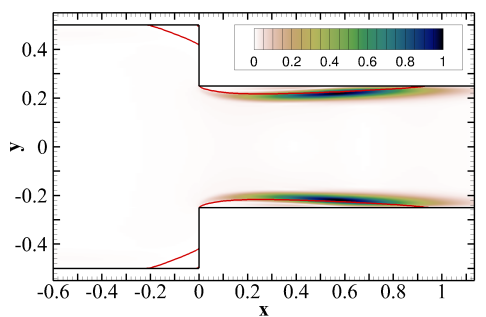

(g) Original structural sensitivity.

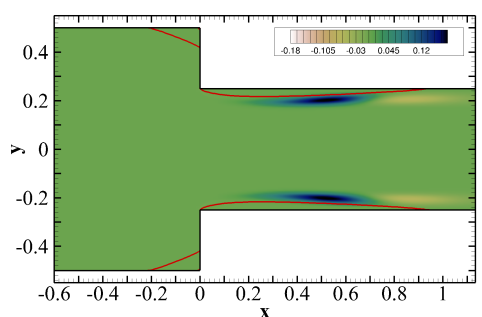

(i) Original lift-up perturbation: $u v d U / d y$.

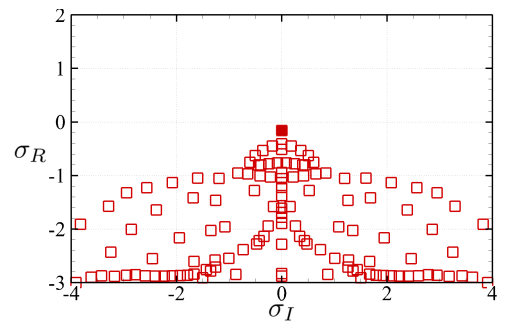

(b) Optimised eigenvalue spectrum.

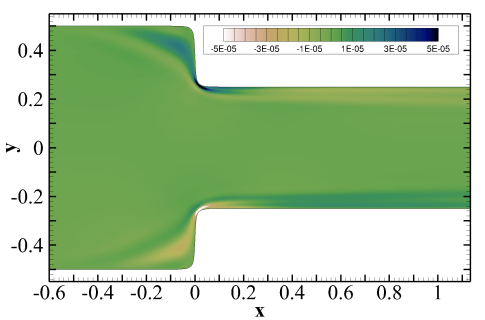

(d) Optimal eigenmodes (horizontal velocity).

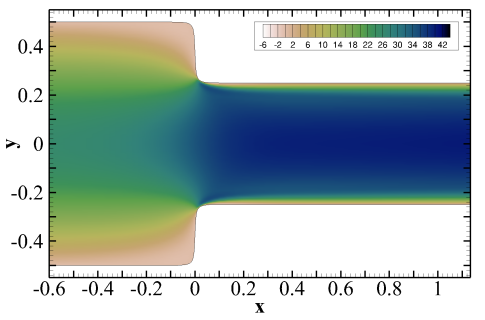

(f) Optimised base flow (horizontal velocity).

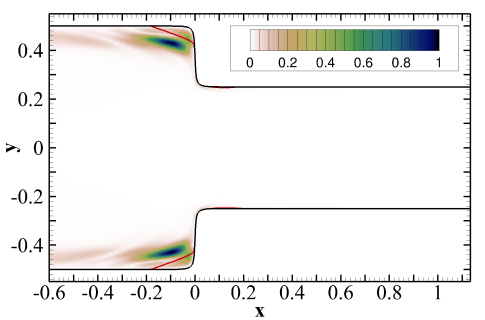

(h) Optimised structural sensitivity.

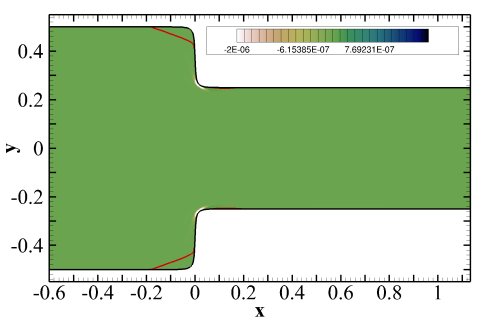

(j) Optimised lift-up perturbation: $u v d U / d y$.

Figure 5.14: Original and optimised geometries for contraction ratio $C=2$ : eigenvalue spectra, eigenmodes, base flows, structural sensitivities and lift-up perturbation kinetic energy terms. Red lines indicate the edge of recirculation regions. 


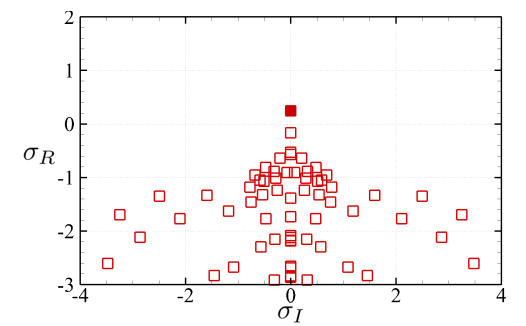

(a) Original eigenvalue spectrum.

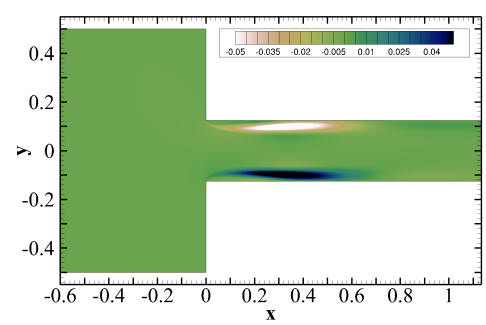

(c) Original eigenmode (horizontal velocity).

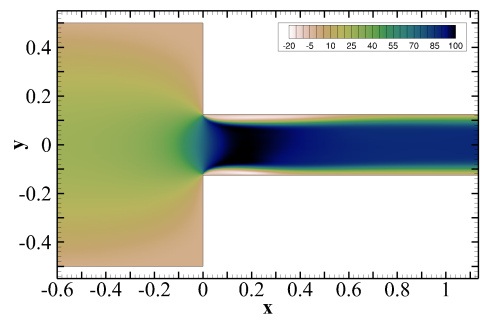

(e) Original base flow (horizontal velocity).

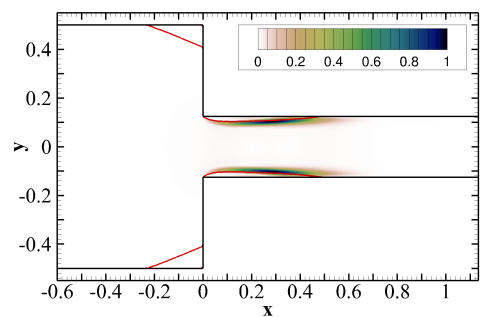

(g) Original structural sensitivity.

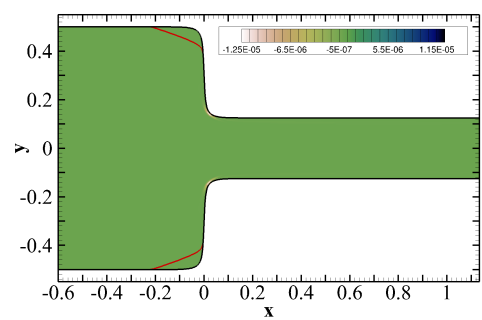

(i) Original lift-up perturbation: $u v d U / d y$.

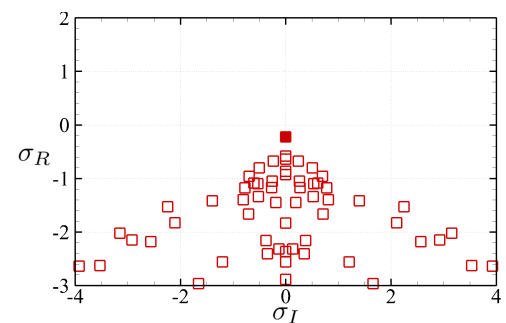

(b) Optimised eigenvalue spectrum.

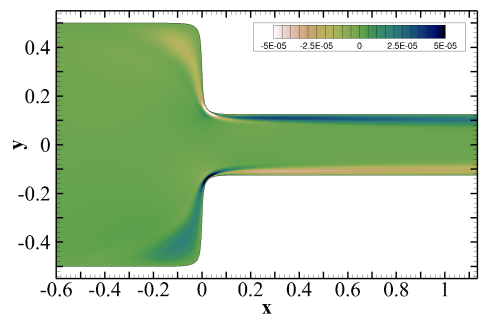

(d) Optimal eigenmodes (horizontal velocity).

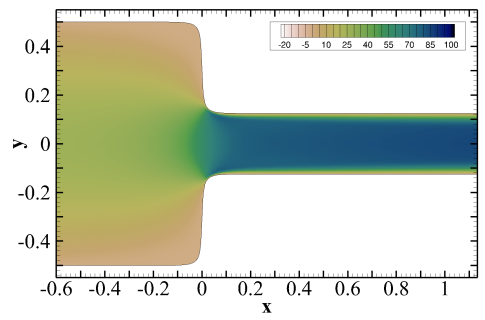

(f) Optimised base flow (horizontal velocity).

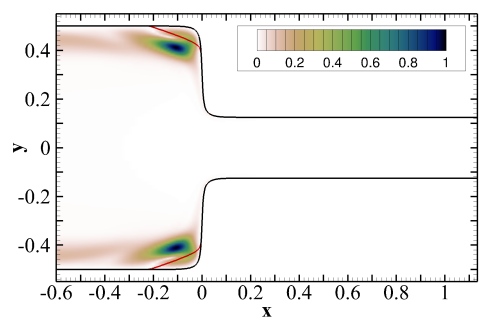

(h) Optimised structural sensitivity.

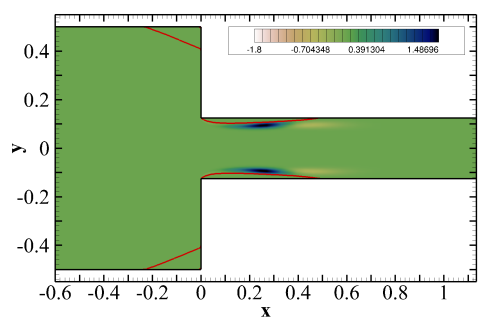

(j) Optimised lift-up perturbation: $u v d U / d y$.

Figure 5.15: Original and optimised geometries for contraction ratio $C=4$ : eigenvalue spectra, eigenmodes, base flows, structural sensitivities and lift-up perturbation kinetic energy terms. Red lines indicate the edge of recirculation regions. 


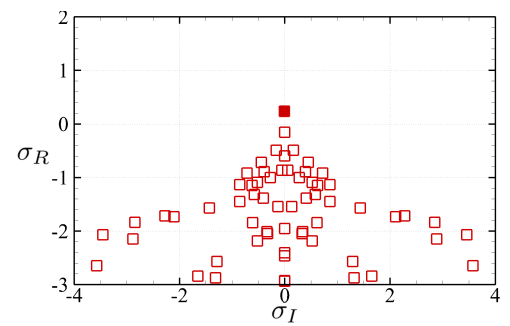

(a) Original eigenvalue spectrum.

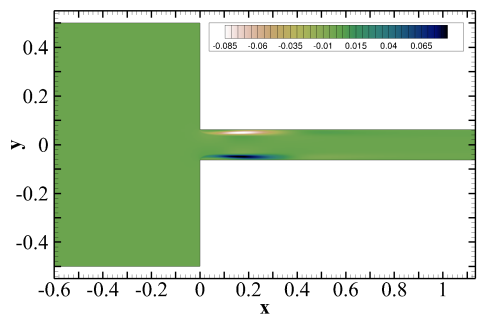

(c) Original eigenmode (horizontal velocity).

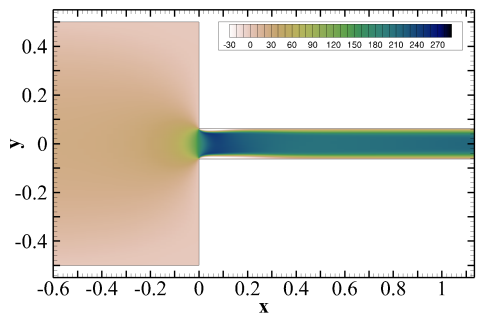

(e) Original base flow (horizontal velocity).

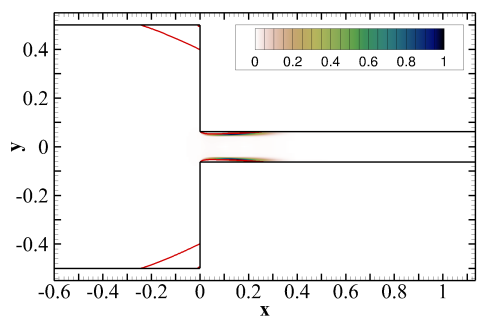

(g) Original structural sensitivity.

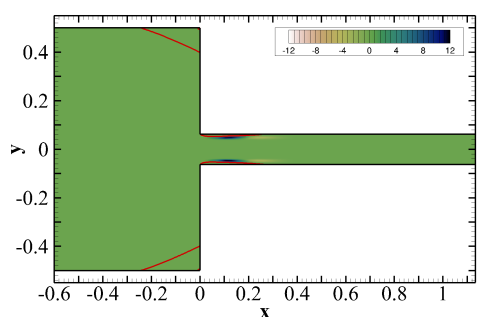

(i) Original lift-up perturbation: $u v d U / d y$.

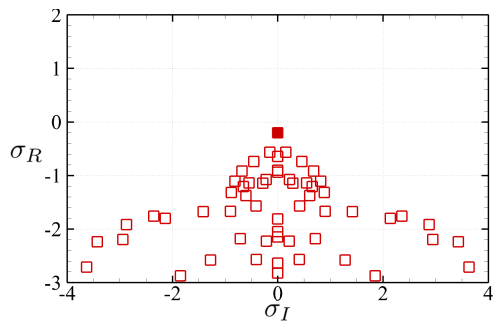

(b) Optimised eigenvalue spectrum.

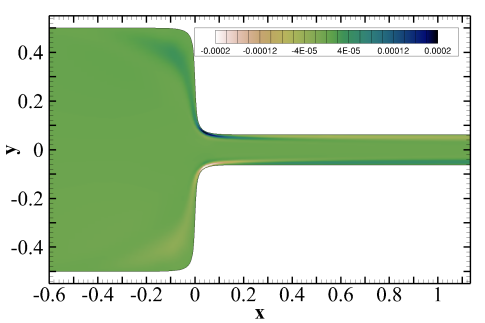

(d) Optimal eigenmodes (horizontal velocity).

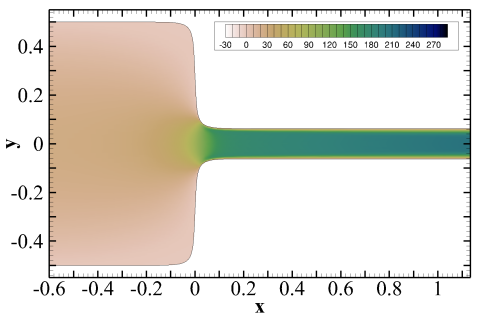

(f) Optimised base flow (horizontal velocity).

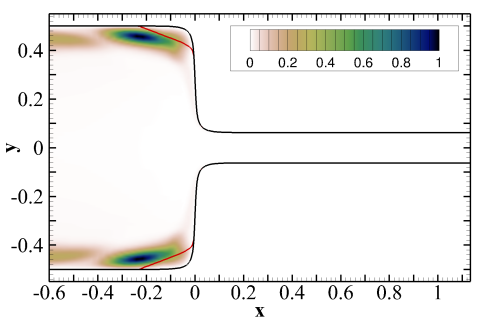

(h) Optimised structural sensitivity.

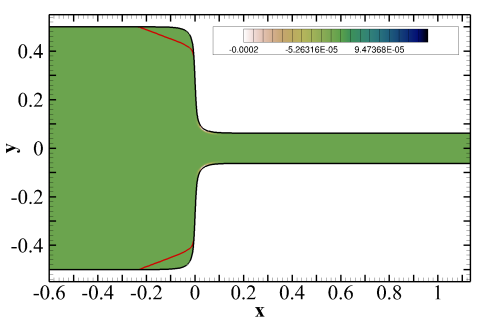

(j) Optimised lift-up perturbation: $u v d U / d y$.

Figure 5.16: Original and optimised geometries for contraction ratio $C=8$ : eigenvalue spectra, eigenmodes, base flows, structural sensitivities and lift-up perturbation kinetic energy terms. Red lines indicate the edge of recirculation regions. 
on the influence of each parameter in the flow stability. We can see for three different contraction ratios that the angle of the contraction stage (vertical walls) affects the growth rate the most whilst the degree of the super elliptic curve at the salient corner affects the least. This parameter-sensitivity analysis suggests that the optimised configurations are optimal within the design space. It is important to note that all optimised configurations reach the edge of the design space, which means that if the design space is enlarged, the optimised configurations may go beyond the current design space. However, we have shown that this design space, which was narrowly restricted to avoid large deformations, is sufficient to enhance flow stability and avoid unsymmetrical flows.

Then, to quantify the increase in critical Reynolds numbers, we include a bifurcation study or the optimised geometries and the three contraction ratios $C=2,4$ and 8 . The size of the recirculation region at the critical Reynolds number has decreased for $C=2$ and 4 . The reattachment lengths against Reynolds numbers of the new shapes of $C=2$ and 4 are shown in figure 5.18.

More interestingly, we can quantify the increase in the critical Reynolds numbers $\left(R e_{c r i}\right)$. For the optimised shapes, these values are $R e_{c r i}=24800$ and $R e_{c r i}=$ 27900 for $C=2$ and 4 respectively. In comparison with the original geometry, in which the critical values were $R e_{c r i}=3150$ and $R e_{c r i}=1420$, the critical Reynolds numbers are vastly increased by 7.9 and 20.9 times for contraction ratio $C=2$ and 4.

Additionally, as for contraction ratio $C=8$, note that there are no signs of bubbles near the tip corners for all the Reynolds numbers tested, which is as high as $4 \times 10^{6}$, shown in figure 5.19 . Thus, the critical Reynolds number is believed not to exist within the range of selected Reynolds numbers.

These results prove that the Reynolds number, at which the bifurcation occur, can be greatly increased through a shape optimisation that includes stability analysis information as the target functional and leads to very small shape deformations.

\subsubsection{The effect of optimisation on the drag reduction}

We can assess the effect of optimisation through the drag, calculated by integrating the forces on all walls. When the Reynolds number is below the critical value, the drag of the full domain channel is exactly the same as twice the drag in the half domain. However, when the Reynolds number is above the critical value, the drag of the full domain channel becomes greater than twice the drag in the half domain. If the Reynolds number is increased, then the ratio between the full domain drag and the doubled half domain drag is also increased.

We choose the channel with contraction ratio $C=4$ as an example to show the relation between drag coefficient and Reynolds number. As shown in figure 5.20, the drag coefficient of both full domain $\left(D_{f}\right)$ and the drag coefficient of doubled half domain $\left(D_{h}\right)$ reduce when the Reynolds number is increasing. Note that $D_{f} / D_{h}=1$ when the Reynolds number is below the critical value. However, when the Reynolds 


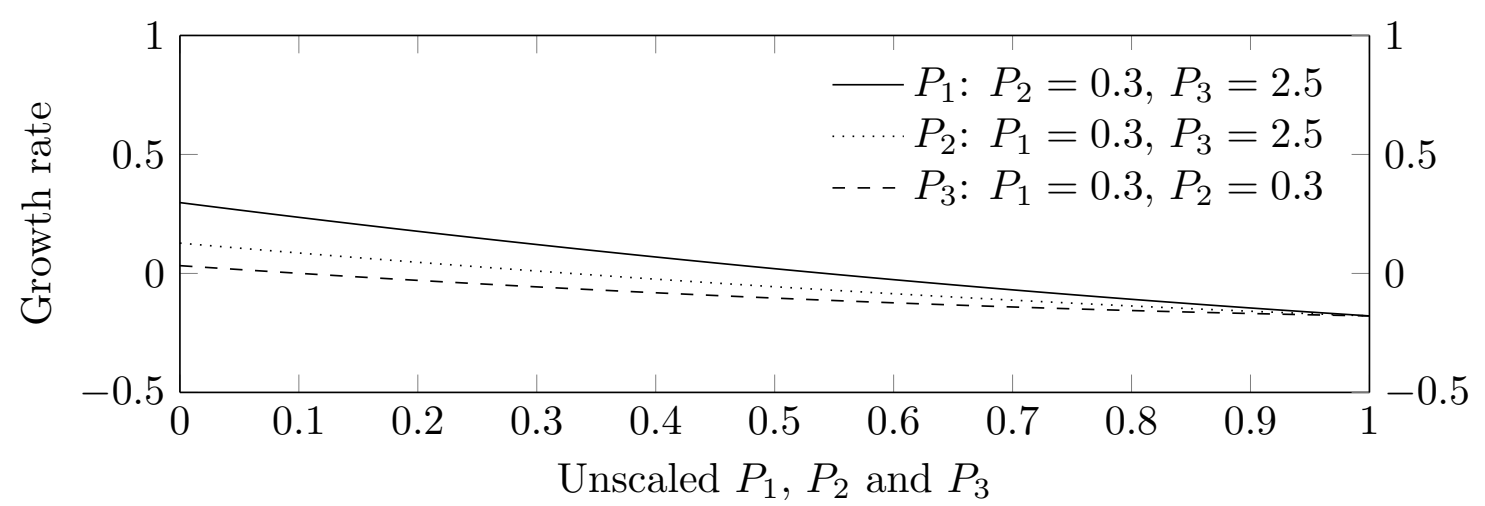

(a) $C=2$.

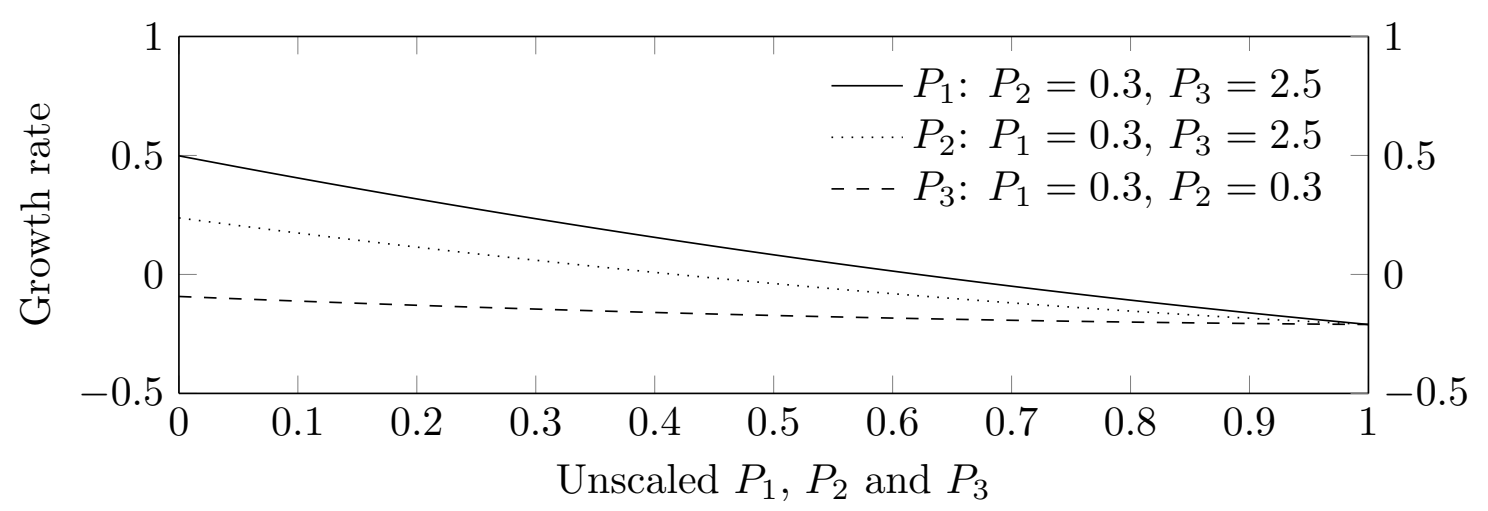

(b) $C=4$.

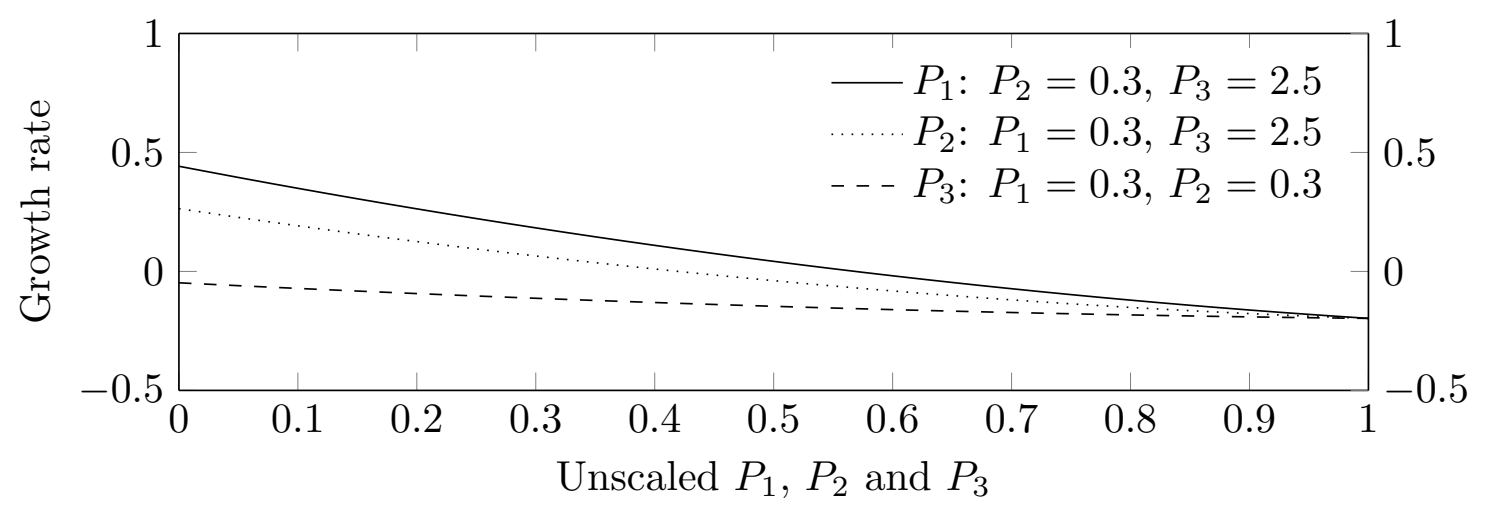

(c) $C=8$.

Figure 5.17: Parameter-sensitivity of the growth rate to variations in the geometric parameters $\boldsymbol{p}=\left(p_{1}, p_{2}, p_{3}\right)$, near the optimised configuration. 


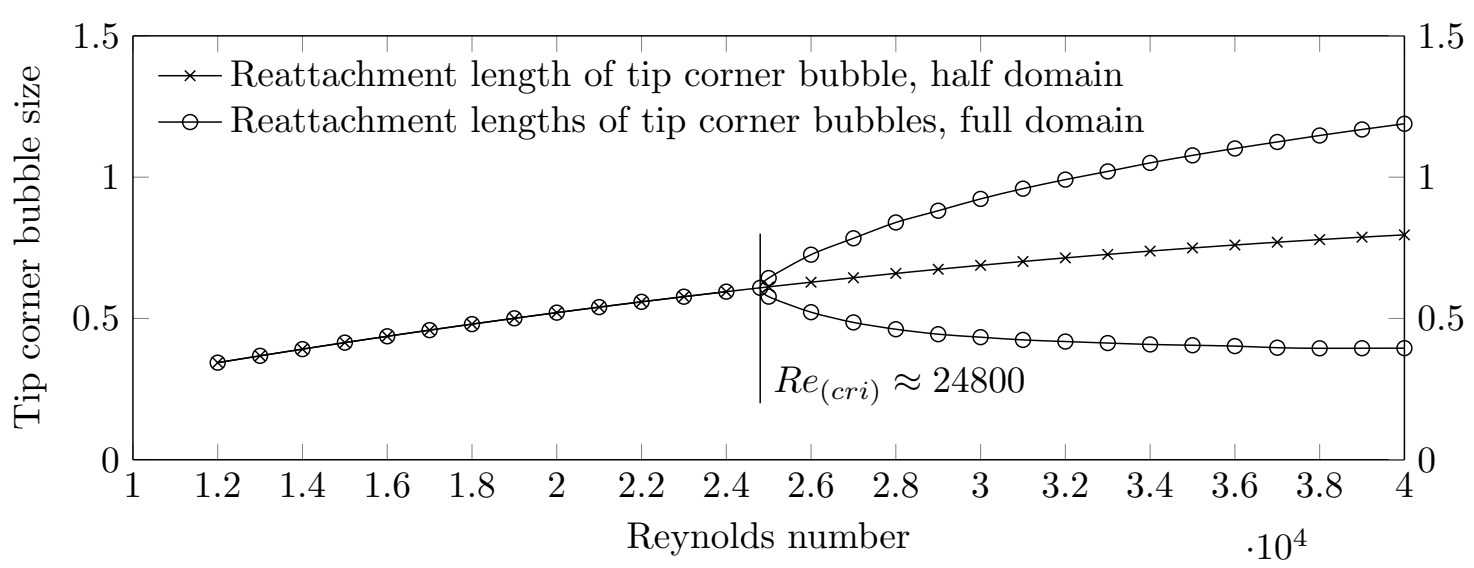

(a) $C=2$.

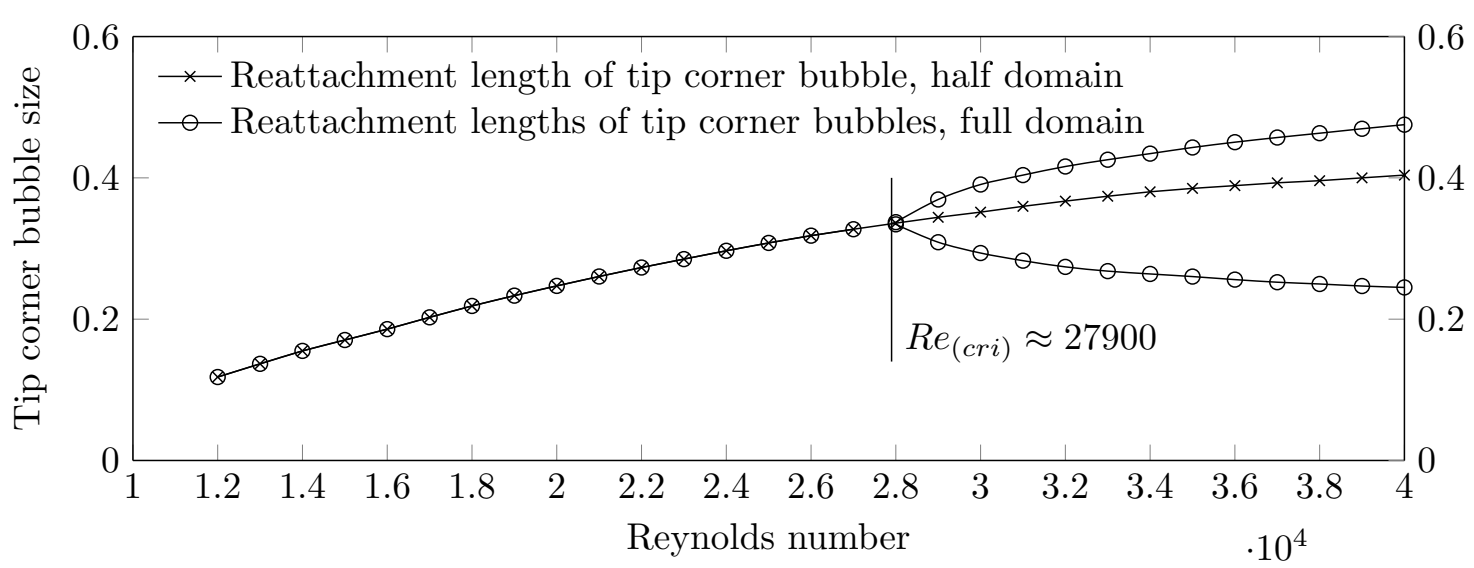

(b) $C=4$.

Figure 5.18: Bifurcation diagram of the optimised geometries for contraction ratios $C=2$ and 4, illustrated using the reattachment lengths $L_{3}$ of downstream tip corner eddy for varying Reynolds numbers.

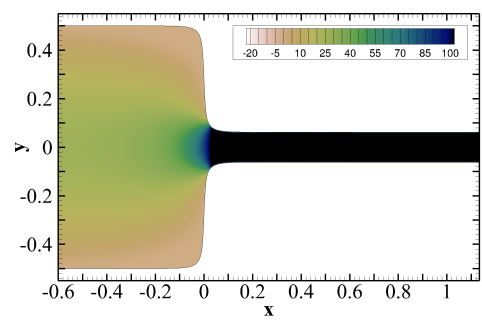

(a) $v_{x}$.

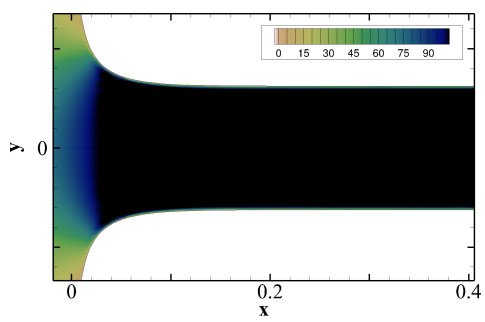

(b) $v_{x}$, zoomed in.

Figure 5.19: Flow field in the optimised geometry, $C=8$. 


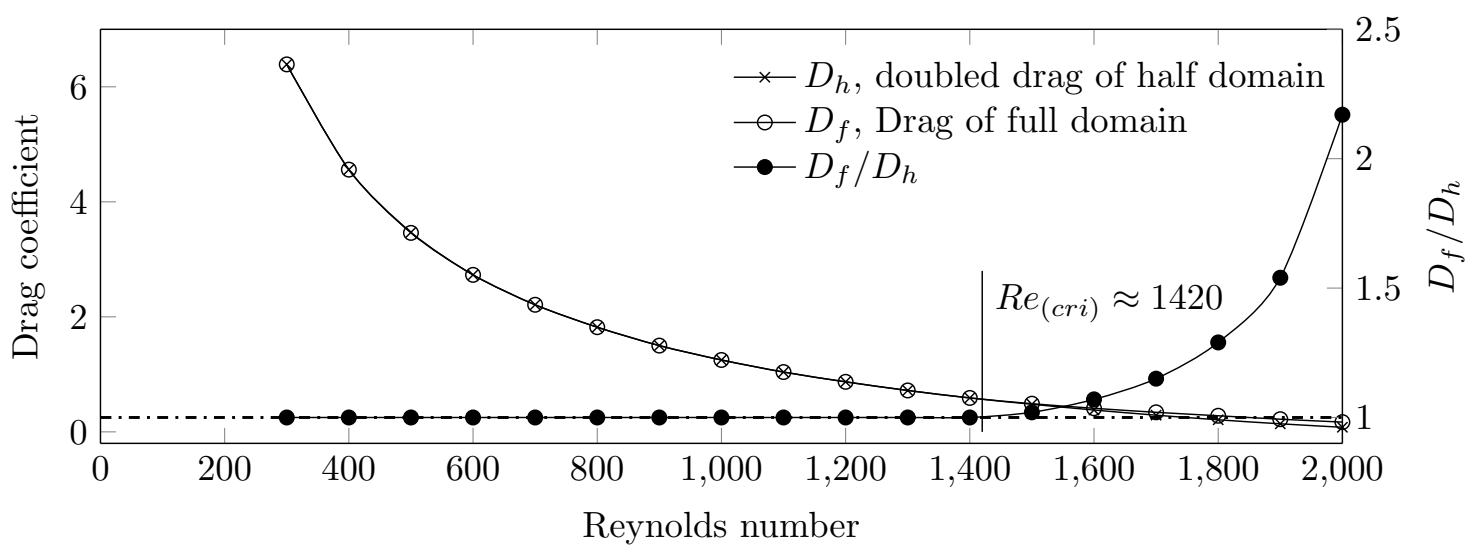

Figure 5.20: The relation between drag coefficient and Reynolds number.

Table 5.9: Drag reduction through shape optimisation.

\begin{tabular}{cccc}
$C$ & $R e_{\text {opt }}$ & $D_{\text {original }}$ & $D_{\text {optimised }}$ \\
\hline 2 & 3200 & 0.116 & 0.042 \\
4 & 1500 & 0.489 & 0.133 \\
8 & 1300 & 1.207 & 0.403
\end{tabular}

number is above $R e_{(c r i)}=1420$, the $D_{f} / D_{h}$ ratio becomes greater than 1 and grows exponentially as the Reynolds number increases.

Then we compare in table 5.9 the drag coefficient before and after the optimisation for the three contraction ratios to find that drag can indeed be greatly reduced by eliminating the bifurcation at the optimisation Reynolds number $\left(R e_{o p t}\right)$. The drag coefficients are reduced by $64 \%, 73 \%$ and $67 \%$ for contraction ratios $C=2$, $C=4$ and $C=8$, respectively.

\subsubsection{Three-dimensional verification on the optimised configuration}

Previously, we have presented the stablisation of the two-dimensional bifurcation by shape optimisation, and showed that the critical Reynolds number can be greatly increased. In this section, we confirm that these results are still valid when considering three-dimensional flows. To this aim, we proceed to examine whether the two-dimensional optimised shape stabilises the three-dimensional configurations, both original and optimised configurations. In this section, we take contraction ratio $C=4$ as an example to examine the optimisation results in three dimensions.

First, we examine the effect of different spanwise channel lengths on the bubble size. Previous studies by Chiang and Sheu[14] and Guevel et al.[37] have shown that the critical value of Reynolds number and the sizes of the bubbles near the salient corners can depend on the spanwise size, for both contraction channels 
Table 5.10: $L_{3}$ against spanwise size, $R e=1600, C=4$.

\begin{tabular}{cc} 
Span & $L_{3}$ \\
\hline two-dimensional & 0.648754 \\
$0.4 \pi$ & 0.672116 \\
$0.8 \pi$ & 0.672113 \\
$1.2 \pi$ & 0.672113 \\
$1.6 \pi$ & 0.672114 \\
$2.0 \pi$ & 0.672114
\end{tabular}

and expansion channels, by examining the bubble sizes with different spanwise lengths of the channel, using walls as boundary conditions on the two side spanwise boundaries.

However, Guevel et al. showed that the bubbles sizes conveyed asymptotically to the two-dimensional sizes as the spanwise length increased, suggesting that threedimensional flows with periodic boundary conditions can lead to results almost identical to two-dimensional. To confirm this, in the present study, we impose periodic boundary conditions on the two spanwise boundaries. We verify the ability of the periodic boundary condition in representing the channel with an infinite span, by comparing the results with different spanwise lengths.

The sizes of the corner bubbles with different spanwise size is shown in table 5.10, with half domain original geometry configuration and contraction ratio $C=4$ at Reynolds number 1600 . We can see that the size of the bubble in twodimensional and three-dimensional simulations are slightly different, and the bubble sizes are bigger in three-dimensional simulations than that in two-dimensional simulations. It is important to note here that when considering in the simulations of different spanwise lengths, the bubble sizes do not vary. We conclude that when using periodic boundary conditions, there are no important three-dimensional effects affecting the bubbles sizes as suggested in Ref. Guevel2018a.

Subsequently, we choose $2.0 \pi$ and $4.0 \pi$ as the spanwise size of the channel (wave number $\beta=\frac{2 \pi}{\text { span }}=1$ and 2 ) and check if there exist a three-dimensional bifurcation occurring below the critical Reynolds number for the two-dimensional bifurcation. We take contraction ratio $C=4$, full domain, $R e=2000$ as an example and show in table 5.11, that the maximum spanwise component of velocity, $\left|w_{\max }\right|$, is very insignificant, and does not affect the bubble sizes.

We then examine the flow in three dimensions to confirm the effect of optimisation on stabilising the flows in the contraction channels. In figure 5.21, we show the bifurcation diagrams for the three-dimensional original geometry with contraction ratio $C=8$ and with a span of $2 \pi$ and optimised geometry with spans of $2 \pi$ and $4 \pi$. We can see in figure 5.21(a) that the critical Reynolds number of bifurcation is 1370 which is slightly smaller than that in two-dimensional original geometry (shown in red in figure 5.21(a)). As shown in Figs. 5.21(b) and 5.21(c), the critical Reynolds numbers are 23900 and 23700, which are similar to each other 
Table 5.11: $L_{3}$ against spanwise size, $R e=1600, C=4$.

\begin{tabular}{cccc} 
Case & span & $\left|w_{\max }\right|(\mathrm{m} / \mathrm{s})$ & $L_{3}$ (upper, lower) \\
\hline Original, $R e=2000$ & two-dimensional & 0.0 & $0.854,0.305$ \\
& $2.0 \pi$ & $7.55 \times 10^{-7}$ & $0.895,0.312$ \\
Optimised, $R e=30000$ & $4.0 \pi$ & $3.63 \times 10^{-6}$ & $0.895,0.311$ \\
& two-dimensional & 0.0 & $0.391,0.295$ \\
& $2.0 \pi$ & $1.14 \times 10^{-6}$ & $0.444,0.228$ \\
& $4.0 \pi$ & $4.90 \times 10^{-9}$ & $0.444,0.228$
\end{tabular}

and also slightly smaller than that in two-dimensional optimised geometry (shown in figure 5.18(b)). Our results suggest that the three-dimensional modes demonstrated in former studies $[14,37]$ are caused by defining the boundary conditions on the spanwise boundaries as walls.

We conclude that the optimisation performed in two-dimensional that enhances the $R e_{c r i}$ is still valid for three-dimensional flow since no three-dimensional effects neglect the advantages of the optimisation.

\subsubsection{Caveats on turbulent flows}

We have presented an optimisation of the contraction channel based on stability analysis that enables a very important increase in the critical Reynolds number that controls the onset asymmetric flow. Additionally, we have shown that the benefits of the two-dimensional optimised geometry hold when considering three-dimensional flows, since no additional three-dimensional bifurcations dominate.

However, the very important increase in critical Reynolds has to be analysed with caution, since other physical mechanisms (e.g. transition due to non-normal transient growth [95]) could change the limiting Reynolds here presented and have not been considered in our study. It may be argued that laminar recirculating bubbles can act as noise amplifier, triggering turbulence transition. Therefore, delaying their appearance (avoiding bifurcations) can delay transition to turbulence. Nevertheless, this is only a particular scenario and other mechanisms (e.g. bypass transition due to non-normal transient growth) can lead to early transition.

In fact, it is likely that transition to turbulence could develop at Reynolds numbers above the original critical Reynolds (not optimised geometry) and below the critical Reynolds of the optimised geometries. Analyses of such features remain out of the scope of this work, but in any case neglect the enhancements in delaying asymmetries showed here.

\subsection{Summary and conclusions}

The following bullet points summarise the main findings included in this work. 


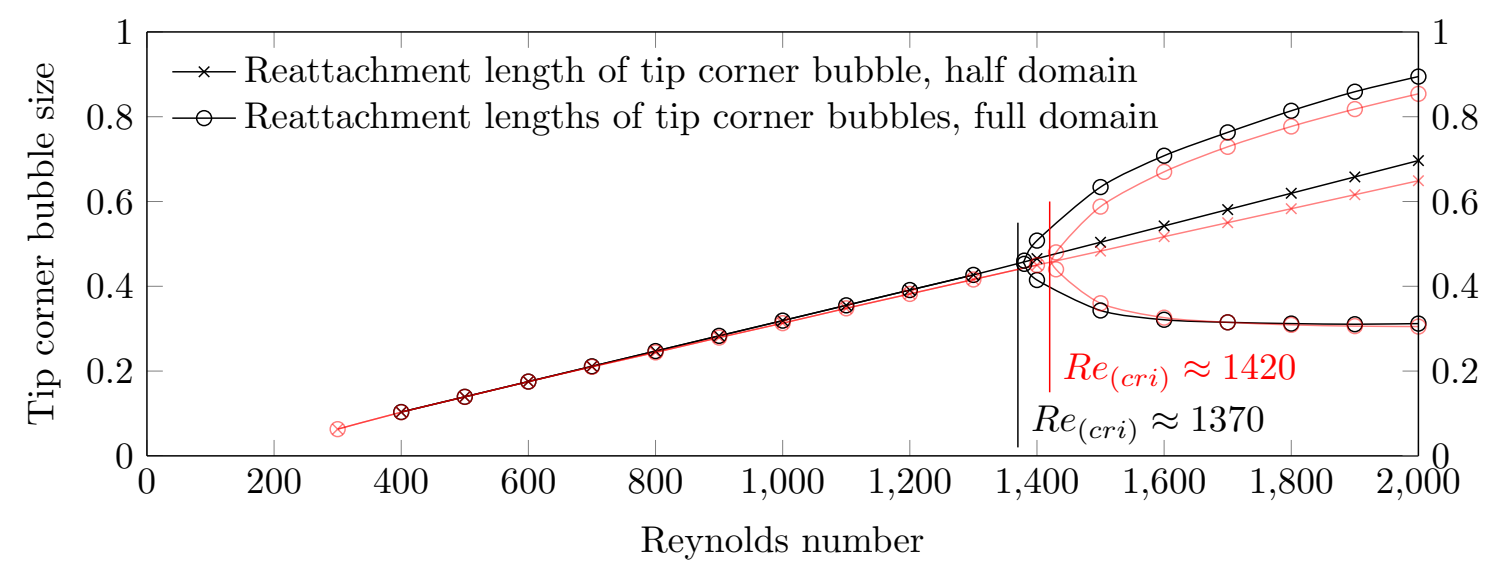

(a) Original geometry, $\operatorname{span}=2 \pi$, (red for 2D).

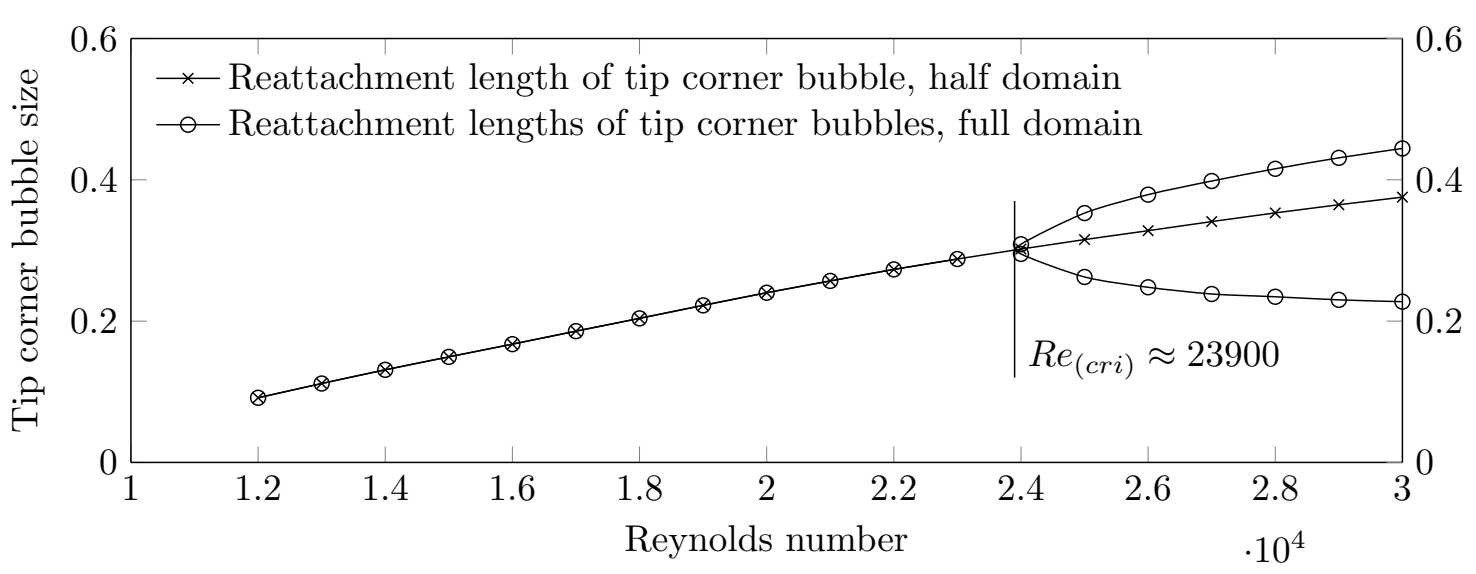

(b) Optimised geometry, $\operatorname{span}=2 \pi$.

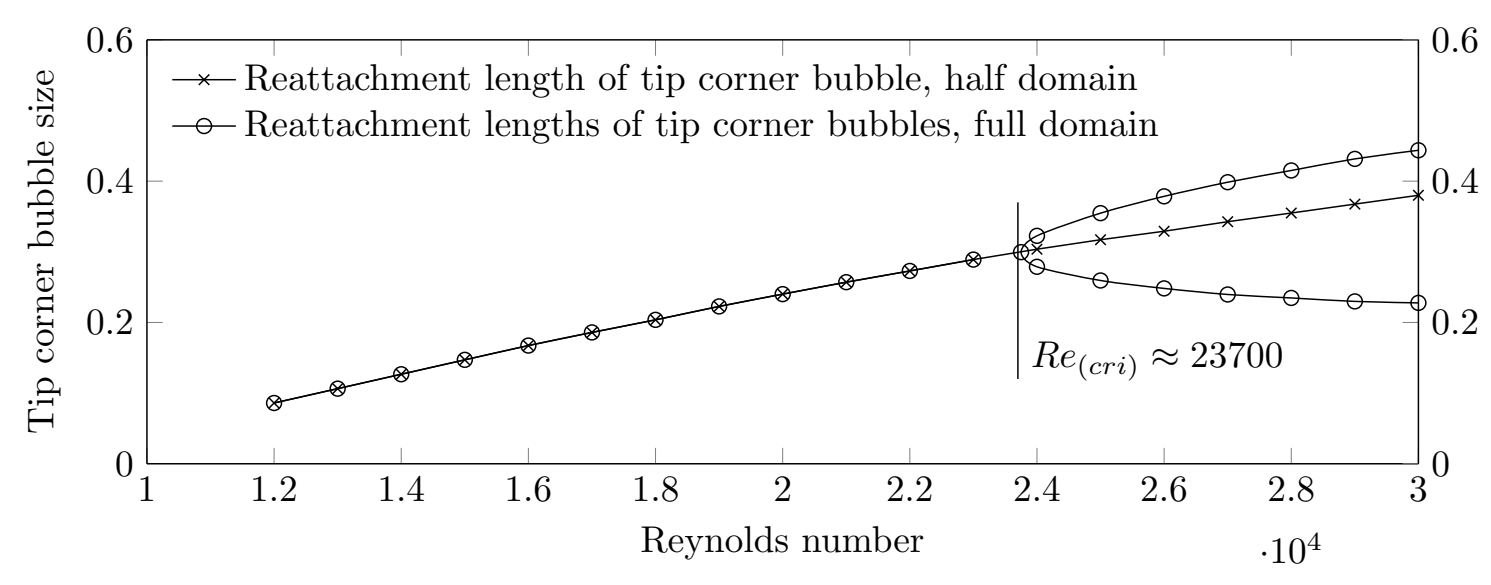

(c) Optimised geometry, span $=4 \pi$.

Figure 5.21: Verification of the results with three-dimensional domain. 
- Computational investigations have been performed to study flow bifurcation in the symmetric planar contraction channel. Flow simulations at different Reynolds numbers for three contraction ratios $(C=2,4,8)$ have been performed and confirm the presence of pitchfork bifurcations, controlled by an asymmetric global mode. Critical Reynolds numbers for all bifurcations are included.

- Stability analysis on the contraction channels relate the eigenmodes responsible for the flow bifurcations to the onset of asymmetries. The instabilities are explained by the lift-up mechanism.

- An optimisation method based on stability analysis is developed for investigations on suppressing the modes responsible for asymmetries. The method consists of an optimiser, a dynamic updating surrogate model, a geometry parametrisation method, a base flow simulator, a stability analysis tool, an eigenmode tracking scheme and a mesh deformation module. This procedure is applied to suppress the asymmetric mode through small shape deformations.

- The optimised geometries show rounded corners and minimise the lift-up physical mechanisms responsible for the instability governing the asymmetric flow. We show that very small modification in the geometry lead to a complete change in the stability, increasing the critical Reynolds numbers of the bifurcations.

- We show that the critical Reynolds number of the original geometry can be increased significantly when optimising the shape. The optimised geometries are stable for Reynolds numbers well beyond the original values: 7.9 and 20.9 times higher for contraction ratios $C=2$ and 4 respectively. The tip corner bubbles are entirely eliminated for contraction ratio $C=8$ for Reynolds numbers as high as $4 \times 10^{6}$.

- The optimised geometry configuration is investigated in three dimensions and confirm that the enhanced stability obtained through the two-dimensional optimisation hold in three-dimensional flows.

- At the optimisation Reynolds numbers $\left(R e_{o p t}\right)$ for each contraction ratios, the drag coefficients are also greatly reduced, by $64 \%, 73 \%$ and $67 \%$ for contraction ratios $C=2,4$ and 8 , respectively.

- In the future, the gradient information of the growth rate to the shape parameters will guide the optimisation investigation through a gradient based approach. 


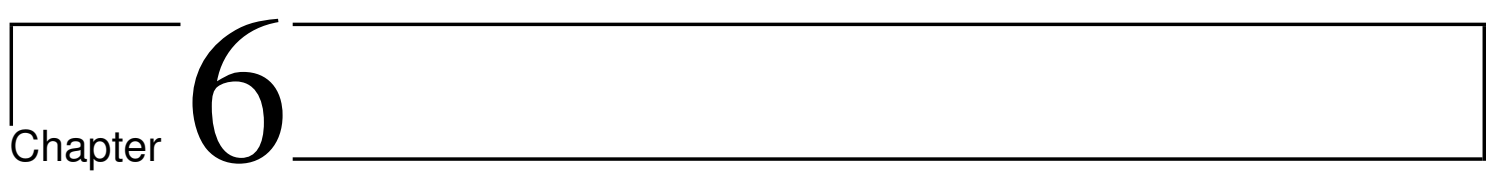

\section{Active flow control optimisation in low-pressure turbines}

\section{Contents}

6.1 Problem description . . . . . . . . . . . . . . 75

6.2 Numerical results $\ldots \ldots \ldots \ldots \ldots \ldots$

6.2 .1 Flow simulation . . . . . . . . . . . . . . 79

6.2.2 Stability analysis and sensitivity analysis . . . . . . 80

6.3 Aerodynamic optimisation to find the optimal flow control strategy .................... 84

6.3.1 Results: 2-parameter optimisation . . . . . . . . 85

6.3.2 Results: 3-parameter optimisation . . . . . . . . . . 89

6.4 Conclusions $\ldots \ldots \ldots \ldots \ldots \ldots \ldots \ldots \ldots \ldots$

In this chapter, the methodology is applied to a low-pressure turbine to seek an optimal active flow control strategy.

Boundary layer separation significantly affects the aerothermal performance of low-pressure turbines in flight vehicles at high altitude. In this investigation, we present the aft portion of the suction side of a low-pressure turbine as a wall mounted hump, where the flow diffusion could lead to boundary layer detachment at low Reynolds numbers and fully attached flow at higher Reynolds numbers. The goal of the current research is to find an effective flow control approach to prevent the boundary layer detachment and reduce dynamic head pressure loss with a minimum energy requirements.

To do that, stability and sensitivity analysis on the flow is conducted. The eigenmode that is causing the boundary layer detachment is identified with BiGlobal stability analysis and with the help of the structural sensitivity, we locate the area where modification in geometry or flow injection and ingestion would have the maximum effect on the evolution of this eigenmode. Assisted with information, we perform an optimisation investigations to find the optimal location and blow rate 
of the injection. The optimal location and blow rate aims to inhibit the separated flow regions exposed to transient mean flow conditions.

In this chapter, we prove the feasibility of finding an optimal flow control strategy that considers the inherent transient behaviour of the flow by optimisation.

\subsection{Problem description}

One specific challenge in designing flight vehicle is the development of an engine that adapts to a large range of Reynolds numbers.

The operating Reynolds number during high-altitude cruise for the low-pressure turbines (LPT) in a flight vehicle can drop drastically compared to the operating conditions at low altitude. However, the development of more compact and versatile distributed-thrust engine has been constrained by the occurrence of flow separation in low-pressure turbine airfoils [7, 34, 77, 89]. At low Reynolds numbers (below the order of $10^{6}$, based on the airfoil chord and inlet velocity), the rear part of the suction side of the airfoil can experience flow separation which increases the pressure loss and reduces the efficiency of the turbine blades, which, therefore affects the performance of the flight vehicle at high altitude.

There are many investigations in the literature on the control of flow separation via both passive and active techniques for the integration of compact low pressure turbine blades.

Passive control techniques require no energy input or complicated actuation mechanisms, which makes it easier to implement. Gurney flaps concepts were introduced by Byerley et al. [11] to control the laminar flow separation on turbine blades. The flaps mounted on the pressure side of the airfoil near the trailing edge are capable of reducing the adverse pressure gradient at low Reynolds conditions by turning and accelerate the flow through the passage, directing it toward the suction side and therefore control the separation on the turbine blades. Other applications can be found in Lake et al. [62], who introduced the use of surface dimples to reduce the size of the separated flow regions. Volino [109] investigated on the effect of rectangular bars on the suction side surface to promote the boundary layer transition and encourage the flow reattachment. However, the added geometrical elements or a deformation based on the original configuration, alter the performance greatly at off-design conditions when the engine operates at higher Reynolds number conditions[48].

Comparing to passive control techniques, active flow control techniques are able to adjust the parameters in the control strategies to adapt to different operating conditions. Greenblatt and Wygnanski [36] documented the control of flow separation by periodic excitation with a hydrodynamic approach, ranging from acoustic wave-based approaches to flow injection and ingestion. The application of glow discharge plasma actuation on the boundary layer control has been experimentally [49] and numerically [80] proven to enhance a fast flow reattachment. 
Flow stability analysis [95] predicts how small flow perturbations grow or decay with respect to an equilibrium flow solution (the base flow), providing information about the onset of the physical mechanisms (reported as modes) responsible for detachments can help to determine means by which to control it. The linear stability community has developed tools to analyse the onset of unfavoured modes, which have been popularised as adjoint-based sensitivity analysis. Sensitivity analysis identifies the regions of the flow that, if modified, lead to the greatest damping (or amplification) of the feature responsible for the instability. The numerical study of flow sensitivity relies on the use of adjoints [66]. The importance of adjoints and sensitivity maps, together with the mathematical tools required for the study of the sensitive flow regions, to different parameters, were first introduced by Hill [45], and have been used extensively to examine the receptivity to internal and external modifications [32, 66, 69], and also by the authors [10, 25, 26, 35, 56]. Having determined the most sensitive flow regions, it becomes relatively simple to apply a flow control technique. Passive control introduces inactive objects (e.g. a small cylinder), which modifies the flow to stabilise or modify the frequency of particular flow features [69]. Useful active flow control may also be derived from sensitivity maps to control undesirable instabilities [98].

In recent years, aerodynamic shape optimisation based on stability analysis has become a valuable tool for the design of efficient lifting surfaces and even entire aircraft [79]. The advances in computing power and development of more accurate computational fluid dynamics solvers have promoted optimal shape design [52]. Examples of the application of this joing methodology can be found in Pralits and Hanifi [83], who introduced a theory for computing the optimal steady suction distribution to suppress convectively unstable disturbances in growing boundary layers on infinite swept wings. Wang [111] coupled adjoint approach with Stakelberg game theory in single- and two-objective aerodynamic optimisation investigations and proved the effectiveness and efficiency of this method in optimisations with complex numerical model and large number of design variables. Wang et al. [112] successfully combined optimisation with stability analysis, and conducted aerodynamic optimisation with minimising the most unstable asymmetric mode to delay the instability and obtained symetric flow for larger range of Reynolds numbers. Properly modelled optimisation approaches have shown their ability in solving complex aerodynamic problems.

In this work, we extend previous research with the investigation and investigate on the detachment recirculation at the wall mounted hump and the difference of the recirculation bubble sizes at different Reynolds numbers. We identify the modes and structures that dominate the unsteady flow behaviour of the recirculated flow region with stability analysis. In addition, we optimise with the growth rate of the most unstable mode as the objective. In the optimisation investigations, we find optimal injection strategies within the designated range of parameters which result in highest total pressure recovery, and optimal injection strategies with the lowest growth rate of the most unstable modes. The results are then compared. 
Numerous experimental and numerical study reveals a recirculation bubble downstream the hump[28, 57, 81]. Further numerical study by Saavedra shows that whilst the Reynolds number increases, the size of the recirculation bubble reduces, which is also the case in low-pressure turbine[46, 86, 102, 110]. The geometry of the channel with a wall-mounted hump is depicted in Figure 6.1. The channel is consisted with an inlet, an exit, an upper wall and a lower wall on which a hump is mounted. A summary of the parameters describing the geometry and charaterising the flow is listed in Table 6.1.

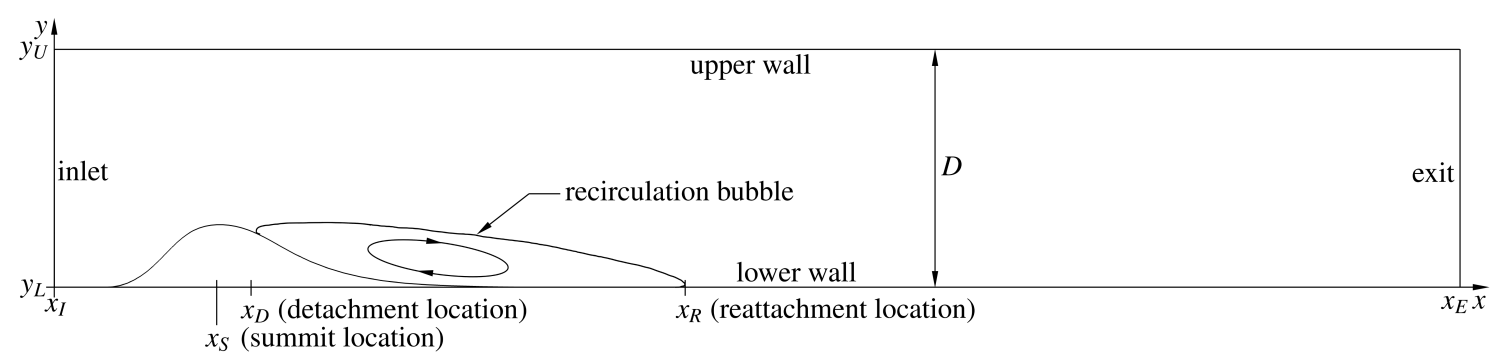

Figure 6.1: Geometric configuration of the channel with a wall-mounted hump.

\begin{tabular}{c|l|c} 
Name & Representation & Value \\
\hline$x_{I}$ & location of the inlet & $0 m$ \\
$x_{E}$ & location of the exit & $1 \mathrm{~m}$ \\
$y_{L}$ & location of the lower wall & $0 \mathrm{~m}$ \\
$y_{U}$ & location of the upper wall & $0.17 \mathrm{~m}$ \\
$D$ & height of the channel $\left(y_{U}-y_{L}\right)$ & $0.17 \mathrm{~m}$ \\
$x_{S}$ & location of the summit of the hump & $0.117 \mathrm{~m}$ \\
$x_{D}$ & detachment location of the flow & \\
$x_{R}$ & reattachment location of the flow &
\end{tabular}

Table 6.1: Parameters describing the geometry and charactorising the flow.

\subsection{Numerical results}

In this section, we discuss the results from the flow simulations, stability and sensitivity analysis and optimisation. Regarding boundary conditions, a steady actuation with total pressure and total density is imposed at the inlet and the injection inlet. The lower wall with the mount hump is set to be a transition wall. A static pressure is imposed on the exit. Since DLR-TAU is a three-dimensional solver, it only deals with three-dimensional mesh. The two spanwise boundaries are set to be symmetry plane for two-dimensional simulation. The $\gamma-R e_{\theta t}$ transition transport model, 
which is detailed in section 2.2.3, is used in the simulations here in this investigation. All the other wall, including the upper wall of the channel and the walls in the injection chamber, are slip walls.

\begin{tabular}{c|c|c} 
Boundary name & Boundary Condition & Notes \\
\hline Inlet & actuation & $P_{0}, \rho_{0}$ \\
Exit & exit-pressure outflow & $P$ \\
Lower wall & transition & \\
Upper wall & slip wall & \\
Symmetry plane & symmetry plane & \\
Injection inlet & reservoir-pressure inflow & $P_{0}, \rho_{0}$ \\
Injection walls & slip wall &
\end{tabular}

Table 6.2: Boundary conditions.

\subsubsection{Flow simulation}

The numerical domain used to model wall mounted hump is displayed in figure 6.2. Where the outlet of the domain is located five axial cords downstream of the test article. At the inlet boundary of the domain pressure and temperature are prescribed together with the turbulent intensity and turbulent length scale. The outlet is modeled as a back pressure boundary where the static pressure level of the simulation is imposed. The top surface is treated as an adiabatic slip line, while the bottom surface is a viscous isothermal wall. The actual total and static quantities imposed in the numerical evaluations are summarized in table 6.3.

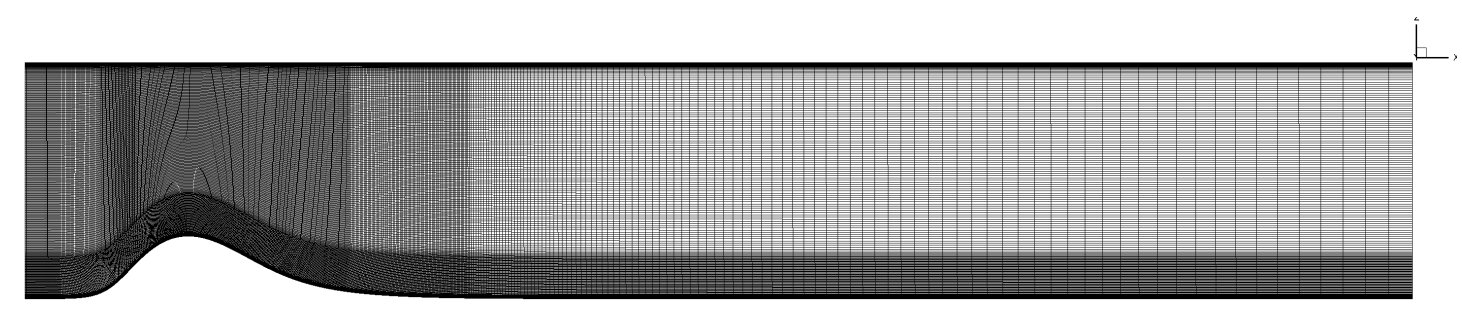

Figure 6.2: Mesh.

The performance of the test article was evaluated at a wide range of inlet total pressure, ranging from 100010 to 110000 corrsponding to Reynolds number from 13980 up to 448759 (based on the chord length of the hump, which is considered as the suction side of a turbine blade, and the mean velocity at the inlet). The detachment and reattachment locations for each one of the pressure differnce between the inlet and the exit explored is shown figure 6.3.

As previously identified for this geometry, the boundary layer detachment inception is insensitive to the Reynolds number and the recirculated flow region starts 


\begin{tabular}{c|ccccc} 
No. & Inlet $P_{0}($ Pascal $)$ & Inlet $T_{0}(K)$ & Exit $P($ Pascal $)$ & Inlet $\rho_{0}\left(\mathrm{~kg} / \mathrm{m}^{3}\right)$ & $R e$ \\
\hline 1 & 100010 & 500 & 100000 & 0.6969 & 13980 \\
2 & 100050 & 500 & 100000 & 0.6972 & 31261 \\
3 & 100100 & 500 & 100000 & 0.6976 & 44214 \\
4 & 100500 & 500 & 100000 & 0.7003 & 98928 \\
5 & 101000 & 500 & 100000 & 0.7038 & 140016 \\
6 & 101649 & 500 & 100000 & 0.7084 & 180000 \\
7 & 105000 & 500 & 100000 & 0.7317 & 315013 \\
8 & 110000 & 500 & 100000 & 0.7666 & 448759
\end{tabular}

Table 6.3: Case list.

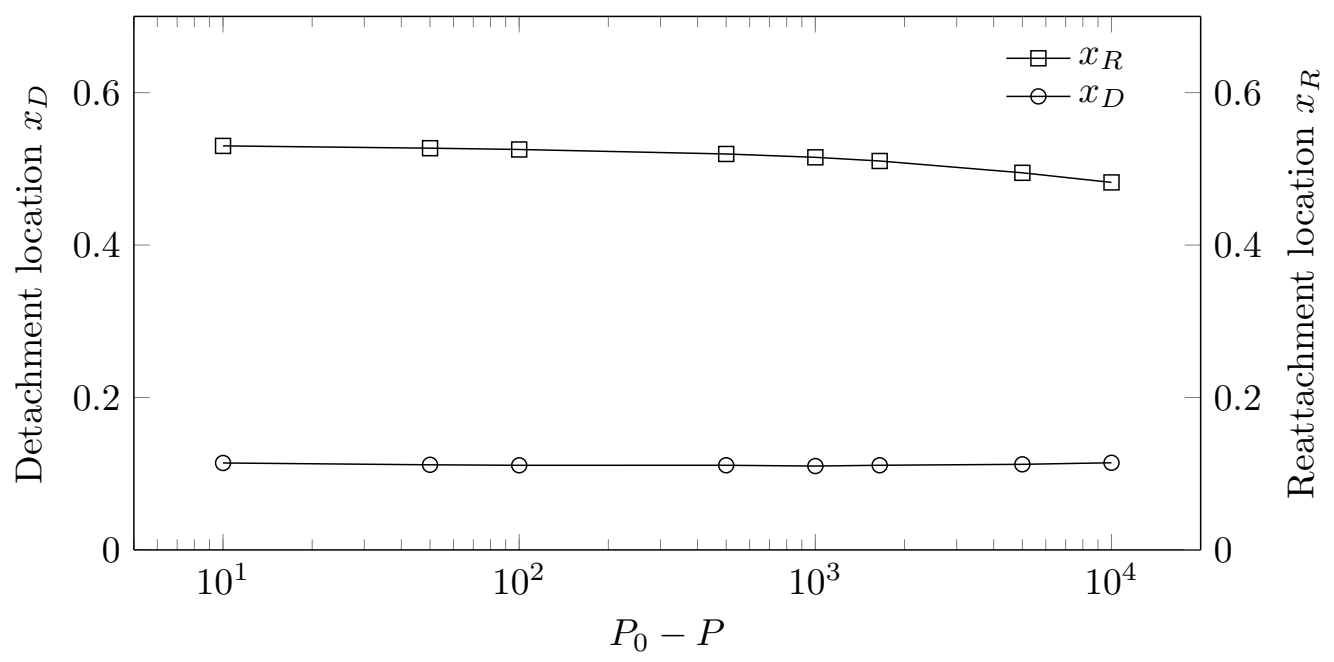

Figure 6.3: Detachment and reattachment locations against inlet total pressure

always in the same location. On the contrary, the reattachment location is determined by the actual Reynolds number. As the Reynolds number decreases the near wall region has less flow momentum to overcome the adverse pressure gradient and its capability to reattach weakens. Consequently, the reattachment location moves upstream as the Reynolds number increases. The correspondent axial velocity contours for each one of the inlet total pressures are shown in figure 6.4. Where the reduction of the recirculated flow region with inlet total pressure can also be identified.

\subsubsection{Stability analysis and sensitivity analysis}

In an attempt to identify the modes and structures that dominate the unsteady flow behaviour of the recirculated flow region an stability analysis is performed. Our goal is to detect the structures that dictate the formation and growth of the detached flow bubble. Once identified, we could introduce flow actuation to alter 

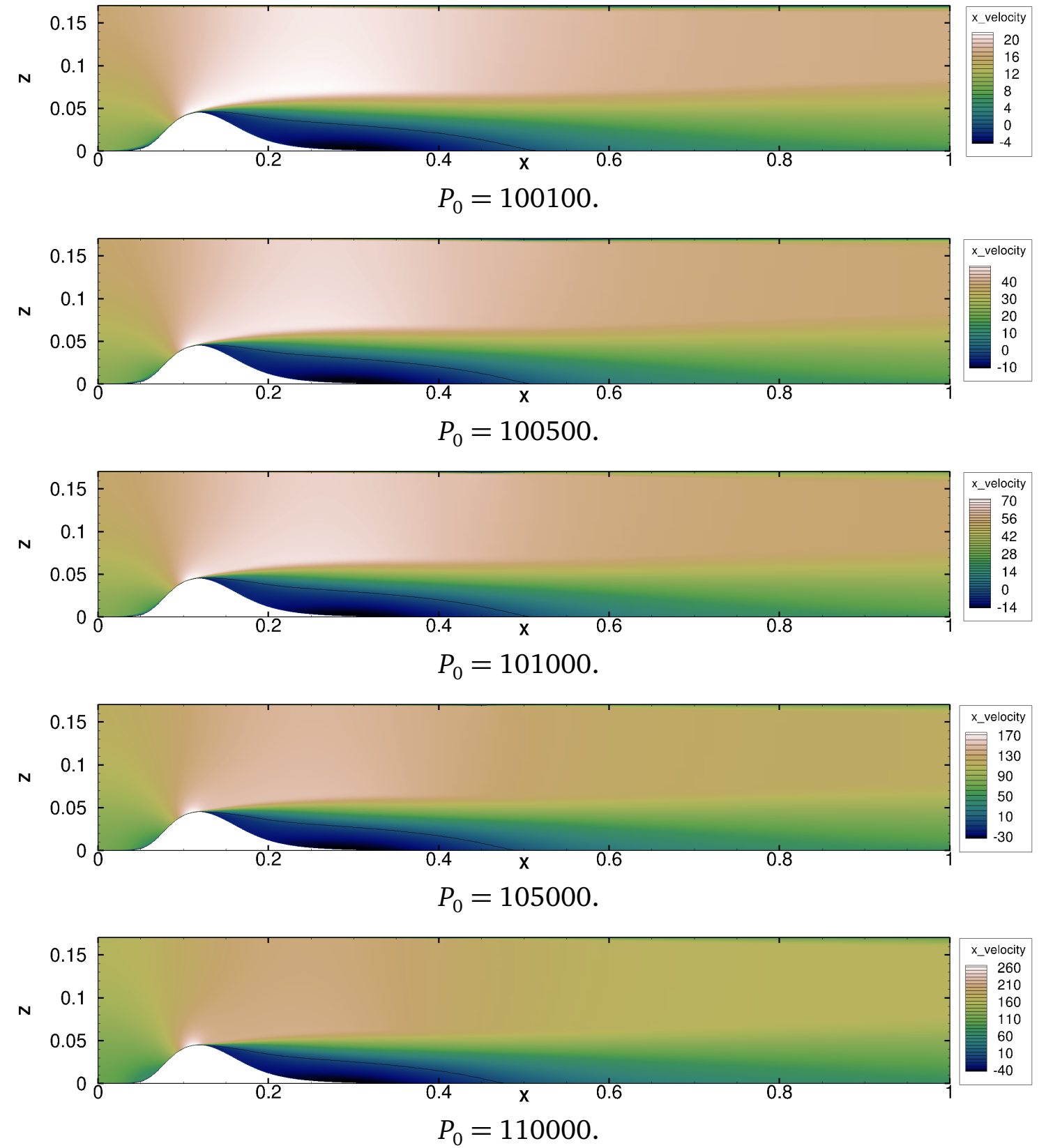

Figure 6.4: $u_{x}$ with different inlet total pressure. 
the performance of separated flow region in an effective manner. Specifically, we carry out stability analyses with inlet total pressure $P_{0}=100500$ without injection, correspondent to a Reynolds number of 98928. The eigenvalue spectrum is shown in figure 6.5. An unstable mode is identified at $\sigma(4.272,0)$. This mode seems to

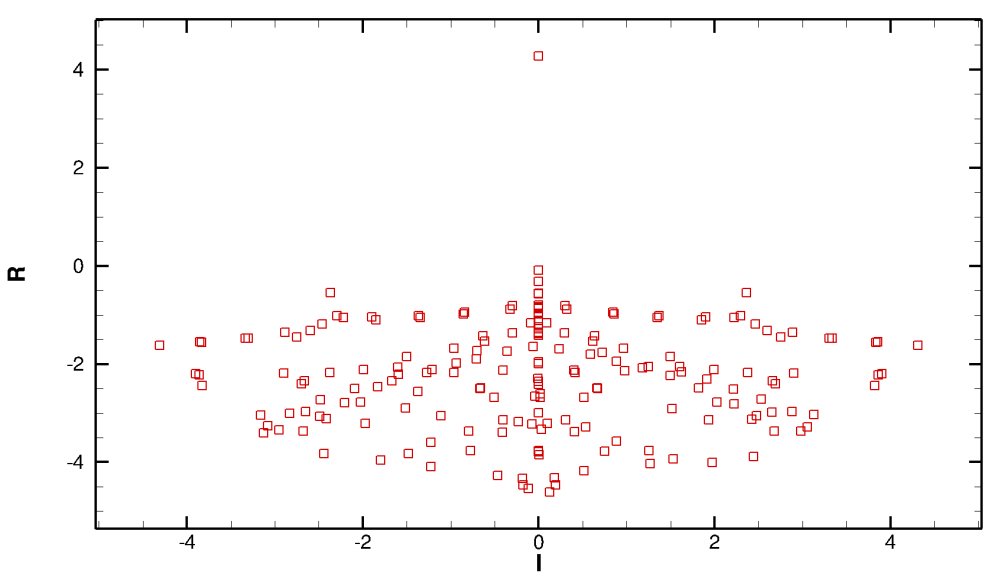

Figure 6.5: Eigenvalue spectrum, $P_{0}=100500$.

dominate the growth of the separated flow region and lift off of the momentum boundary layer from the surface. The mode structure is shown in figures 6.6. Figures $6.6 \mathrm{a}$ and $\mathrm{b}$ represent the axial and normal velocity components representative for the most unstable mode. While figures $6.6 \mathrm{c}$ and $\mathrm{d}$ represent the density and structural sensitivity map respectively. The axial and normal velocity and density identify the maximum strength of the mode right at the boundary layer detachment inception. While the sensitivity contour depicts region of influence of the boundary layer detachment mechanism. These results suggest that for an effective mitigation of the boundary layer detachment the actuation should be located at the origin displayed in the axial velocity contours.

The rest of the modes in the domain do not depict and unstable growth, however the mode at $\sigma=(-0.5436,2.366)$ results of interest due to its proximity to the unstable threshold. The velocity, density and sensitivity map correspondent to this mode are displayed in figure 6.7. The origin of this mode is also located slightly downstream of the hump summit, close to the separation inception and its region of influence extends across the recirculated flow region. Hence any alteration of this mode could eventually affect the growth of the recirculated flow region.

The stability analysis seem to conclude that an effective actuation to prevent the detachment of the boundary layer or reduce the extension of the flow region must right downstream of the hump summit. In this work, we propose to control the detachment by injecting further flow momentum to energize the near wall region and overcome the adverse pressure gradient. 


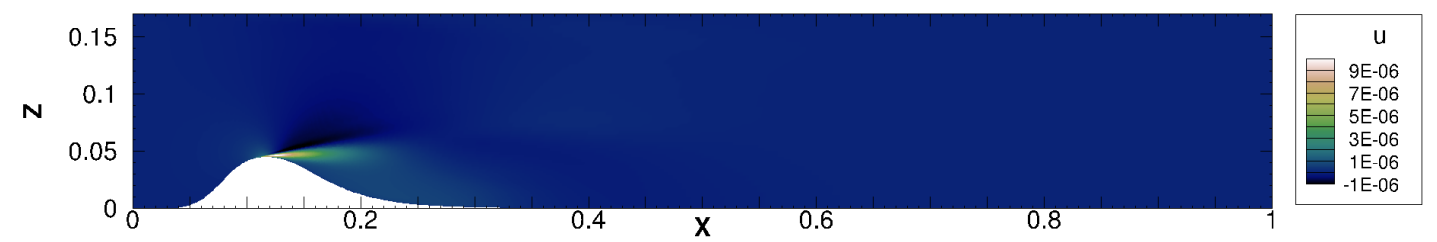

Eigenmode, $u_{x}$.

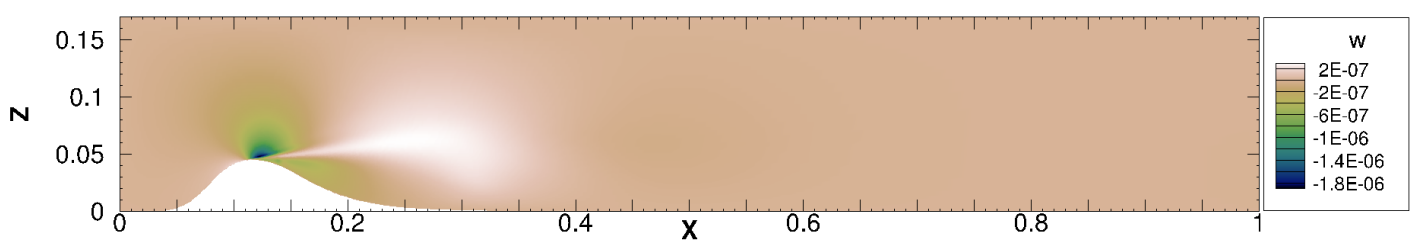

Eigenmode, $u_{y}$.

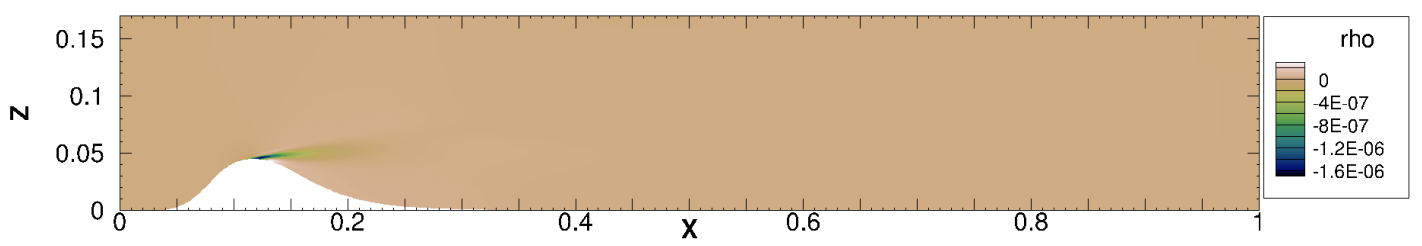

Eigenmode, $\rho$.

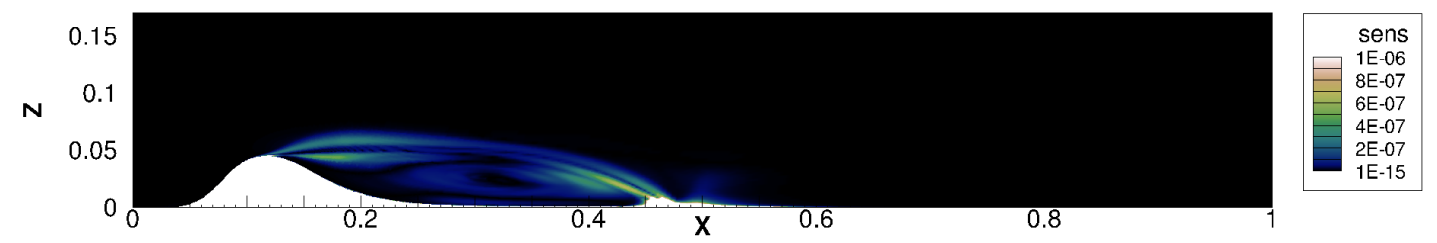

Structural sensitivity map.

Figure 6.6: Eigenmode and its sensitivity map corresponds to $\sigma=(4.272,0)$. 


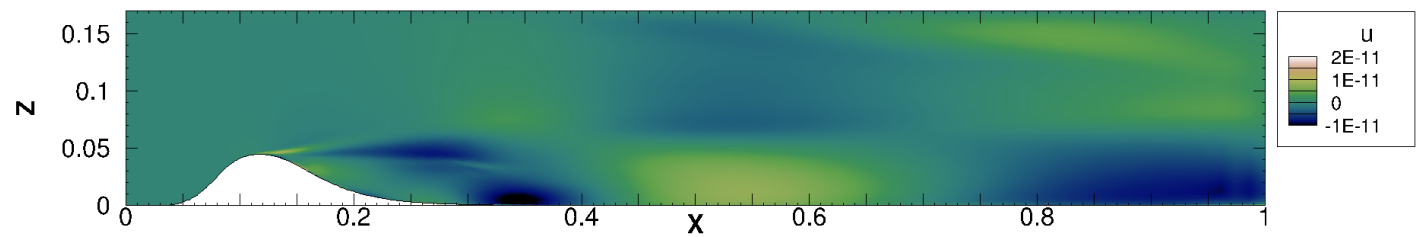

Eigenmode, $u_{x}$.

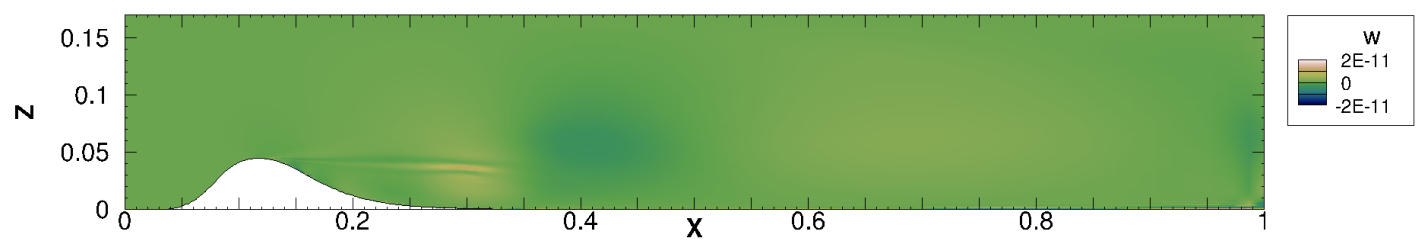

Eigenmode, $u_{y}$.

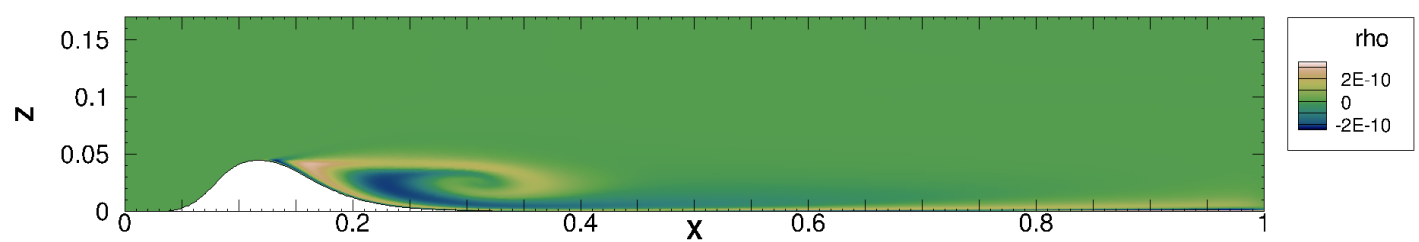

Eigenmode, $\rho$.

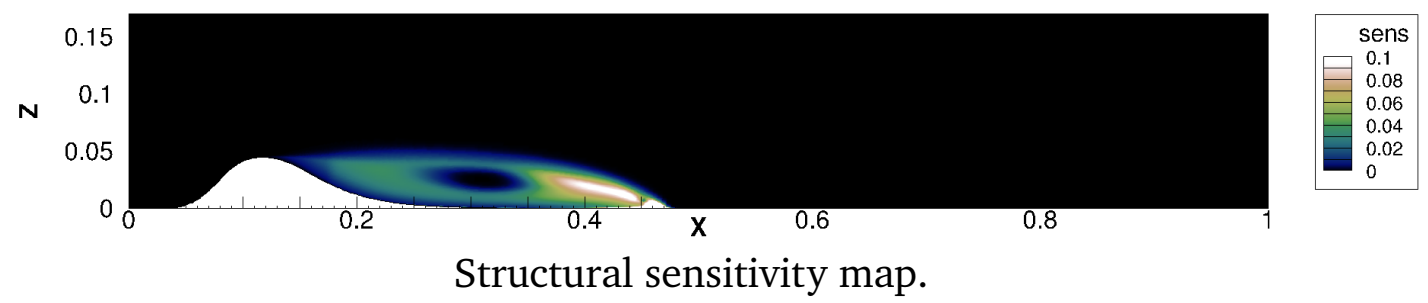

Figure 6.7: Eigenmode and its sensitivity map corresponds to $\sigma=(-0.5436,2.366)$. 


\subsection{Aerodynamic optimisation to find the optimal flow control strategy}

We will here compare a classic optimisation based on pressure loss and an optimisation based on the growth rate provided by the stability analysis. In this investigation, we seek to find an optimal location, optimal injection angle and blow rate to eliminate the recirculation bubble and to maximize the total pressure recovery, and also to find another set of optimal parameters to minimise the growth rate of the most unstable mode. Two different optimisation strategies will be developed and compare, namely minimising pressure losses or eigenvalue amplification:

$$
\begin{array}{cl}
\min : & P_{\text {loss }}(\boldsymbol{p}), \\
\text { subject } \text { to }: & \mathscr{M} \frac{\partial \boldsymbol{q}}{\partial t}=\mathscr{F}(\boldsymbol{q}),
\end{array}
$$

or

$$
\begin{aligned}
& \min : \sigma_{R}(\boldsymbol{p}), \\
& \text { subject } \quad \text { to }: \mathscr{M} \frac{\partial \boldsymbol{q}}{\partial t}=\mathscr{F}(\boldsymbol{q}), \\
& \mathscr{J}(\overline{\boldsymbol{q}}) \hat{\boldsymbol{q}}=\sigma \mathscr{M} \hat{\mathbf{q}},
\end{aligned}
$$

in which, $P_{\text {loss }}$ is the dynamic pressure loss, $\sigma_{R}$ is the growth rate of the most unstable mode, and $P_{0}$ is the total pressure at the inlet of the channel. $R_{b}=$ $\left(\frac{P_{0, \text { injection }}}{P_{0, \text { inlet }}}-1\right) \times 100 \%$ is the blow rate at the injection, $P_{0, \text { injection }}$ is the total pressure at the injection inlet. The dynamic pressure loss, $P l$, is defined by

$$
P_{\text {loss }}=\frac{\frac{\int_{\Omega_{\text {Inlet }}} \dot{m} P_{0}+\int_{\Omega_{\text {Injection }}} \dot{m} P_{0}}{\int_{\Omega_{\text {Inlet }}} \dot{m}+\int_{\Omega_{\text {Injection }}} \dot{m}}-\frac{\int_{\Omega_{\text {Exit }}} \dot{m} P_{0}}{\int_{\Omega_{\text {Exit }}} \dot{m}}}{\frac{\int_{\Omega_{\text {Inlet }}} P_{\text {dyn }}}{\int_{\Omega_{\text {Inlet }}}}} .
$$

where the massflow average total pressure is compared to the inlet massflow average total pressure, $\dot{m}$ is the mass flow $P_{0}$ is the total pressure and $p_{d y n}$ is the dynamic pressure. In addition, to focus the figure of merit on the aerodynamic viscous loses the injected fluid is subtracted from the outlet massflow average quantity.

In this investigation, we include optimisations with two and three parameters to characterise the injection separately. The two-parameter optimisation considers the injection location and the blow rate as the optimisation parameters to seek the optimal injection configuration. In addition to that, the three-parameter optimisation considers another parameter, the angle of the injection. In the two-parameter optimisation, we adopt a full-factorial design of experiments, while in the threeparameter optimisation, we choose a diagonal design to reduce the computational cost. 


\subsubsection{Results: 2-parameter optimisation}

In order to explore the impact of the flow injection along the downstream section of the hump we introduce an injection on the aft part of the hump. The numerical domain is depicted in figure 6.8, The main flow boundary conditions remain unaltered, except for the injection. The massflow injection through the tiny "hole" is controlled by prescribing a total pressure of the injection cavity. $x_{b}$, as shown in Figure 6.8, is the injection location.

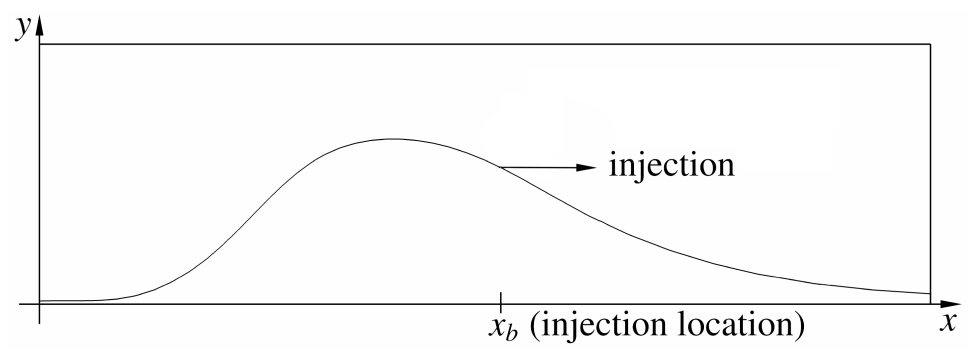

Figure 6.8: Geometric configuration used in the optimisation (zoomed in near the hump), 2-parameter.

In this investigation, we optimise separately aiming on minimising the pressure loss (as given in equation 6.1) and on minimising the amplification rate of the most unstable mode to delay the detachment (as given in equation 6.2). In equations 6.1 and $6.2, \boldsymbol{p}$ is the array of parameters which are $x_{b}$, the $\mathrm{x}$-axial location of the injection.

As applied to the investigations in this section, $P_{\text {loss }}(p)$ is the pressure loss, and $\sigma_{R}(p)$ is the growth rate (real part of the eigenvalue) both governed by the geometry parameter array $\boldsymbol{p}=\left(x_{b}, R_{b}\right)$. The surrogate model is assisted with a uniform full factorial design of experiment for higher precision and with the complexity of the problem considered. We select 7 and 9 levels for optimisation parameters $x_{b}$ and $R_{b}$, respectively. The details of the design of experiment is shown in table 6.4.

Table 6.4: Design of experiment.

\begin{tabular}{c|c|c|c} 
Parameter & Number of levels & Minimum & Maximum \\
\hline$x_{b}$ & 7 & 0.138 & 0.175 \\
$R_{b}$ & 9 & 0 & 20
\end{tabular}

\subsubsection{Optimisation aiming on reducing the pressure loss}

The objective of this optimisation invetigation is to reduce the pressure loss at the outlet of the domain through the mitigation of the recirculated flow region through flow injection (modelled as in equation 6.1). The location of the injection $x_{b}$ and the blow rate $R_{b}$ that is controlled by the inlet total pressure at the injection are the 
main control parameters for the algorithm. The identified optimal configuration resides on actuation at $x_{b}=0.138$ with a blow rate $R_{b}=10.43 \%$, with a total pressure loss, $P_{\text {loss }}=0.575 \%$. The flow field correspondent to such structure are shown in figure 6.9.
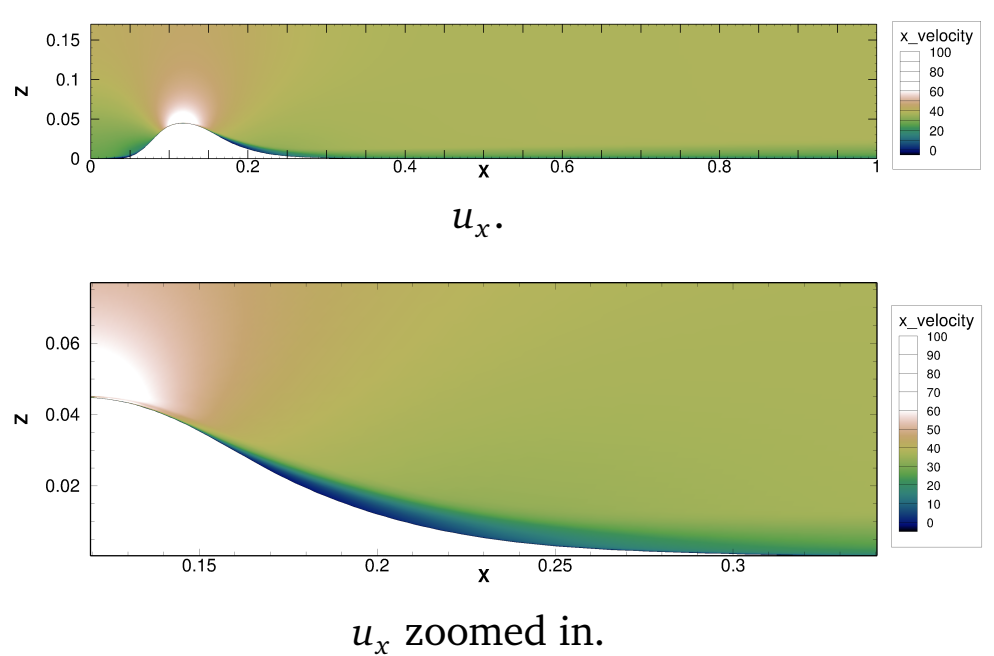

Figure 6.9: $u_{x}$, optimal design, 2-parameter, objective: pressure loss.

The optimization results further support the stability analysis performed where the optimum point of actuation sits in the furthest upstream boundary of our design space, slightly downstream of the hump summit and right before the boundary layer detachment. As identified through the most unstable mode description, the actuation at this location heavily influences the boundary layer detachment process and once the injection pressure ratio provides enough momentum boost, the flow is able to remain attached and the viscous loses are mitigated. Considering the manufacturing complexity the identified optimum injection location also results in the most complicated design where the thickness between the hump summit and injection slot will be minimum. However, taking advantage of the surrogate model, figure 6.9, we can also identify further downstream location that could have still a deep impact on the pressure loses reduction.

The result is further varified with stability analysis. As in shown in figure 6.10, is the eigenspectrum of the optimal configuration of the injection. We can see, and compared to the original eigenvalue spectrum without injection, that no eigenvalue has crossed the $\mathrm{x}$-axis, i.e. all the related eigenmodes have amplification rates below zero, making them stable. It is clear that the optimisation that minimises the pressure loss in the passage has also kept the most unstable mode stable. In figure 6.11, the most unstable eigenmode is shown, from which, we can see that the mode with the optimised injection configuration has a small part where the $x$ component of the velocity perturbation near the wall at the aft portion of the hump that is negative, which could cause a recirculation in the domain, if its amplification rate is above zero. The structural sensitivity map is shown in figure 6.12, in which 
we can see that the most sensitive region in the domain has been altered from detachment and reattachment locations as shown in figure 6.6 to a small region that is near the wall at the aft portion of the hump.

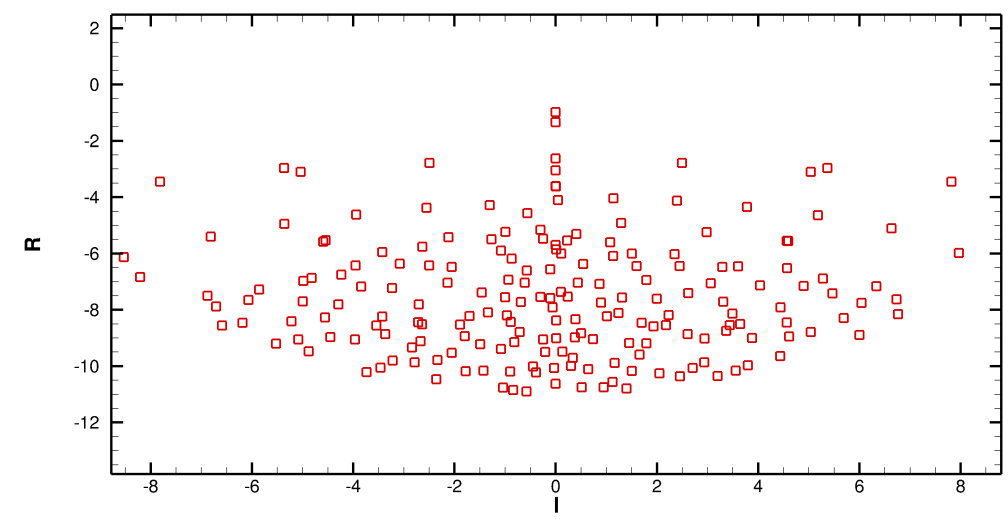

Figure 6.10: The eigenvalue spectrum of the optimal configuration, 2-parameter, objective: pressure loss.

\subsubsection{Optimisation aiming on delaying instability}

In this part, we introduce an optimisation investigation which has the same set of optimisation parameters, same surrogate modelliing and same design of experiments. The only part that is different in this section is that we adopt equation 6.2 as the optmisation model. Instead of reducing the pressure loss, in this section, the optimisation objective is to minimise the amplification rate of the most unstable mode that causes the detachment, for comparing the results of two optimisation investigations with different objectives.

The identified optimal configuration is injection with parameters, $x_{b}=0.139$ and $R_{b}=20 \%$. The location of the injection from this optimisation is not very different than that in which the optimisation objective is minimising the pressure loss. The blow rate, on the other hand, reaches the edge of the design space, specified in table 6.4.

First, let us take a look at the eigenspectrum, as shown in figure 6.13. We can see that the amplification rates of all the modes are kept below zero. Furthermore, the most unstable mode in this investigation has a even smaller amplification rate than that in the optimisation where the objective is minimising the pressure loss.

The eigenmodes are shown in figure 6.14. We can see that, with the strong injection, the most unstable mode is relocated to be in the uptream side of the hump. The structual sensitivity map of this mode is shown in figure, we can see that the optmisation has altered the most sensitive location of this mode is also altered to the front of the hump. In figure 6.16, we show the $u_{x}$ component of the 

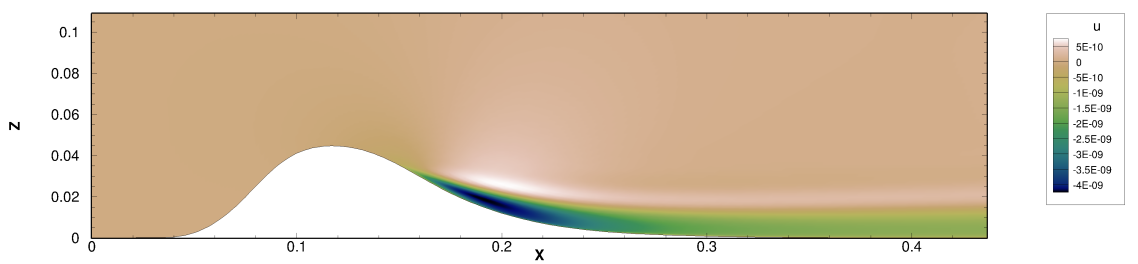

$u$.

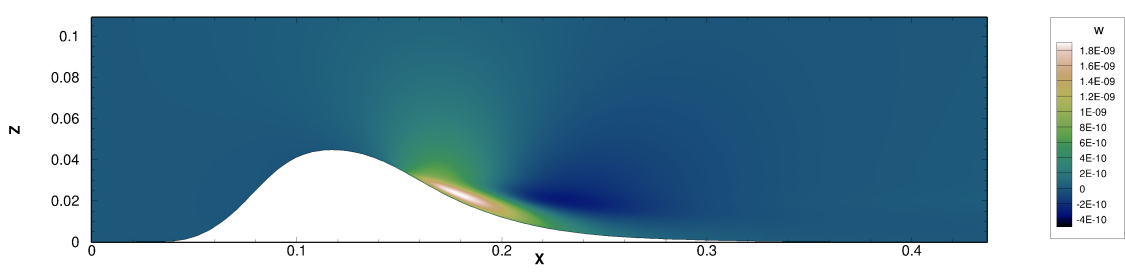

$w$.

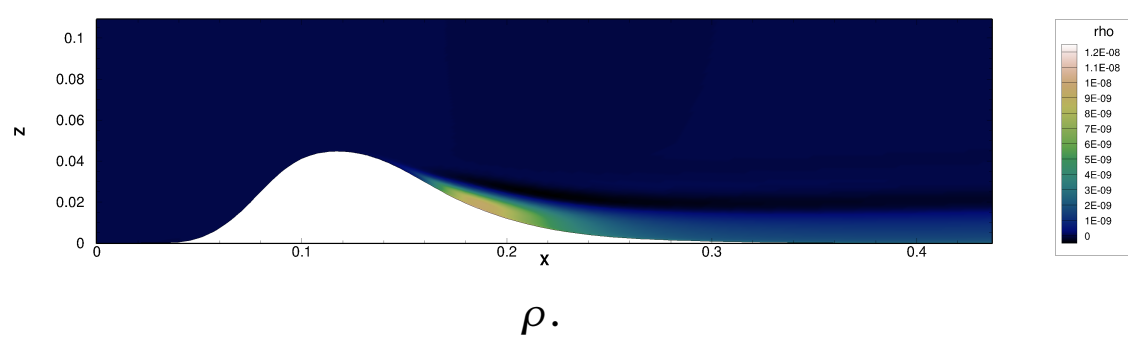

Figure 6.11: The most unstable eigenmode of the optimal design, 2-parameter, objective: pressure loss.

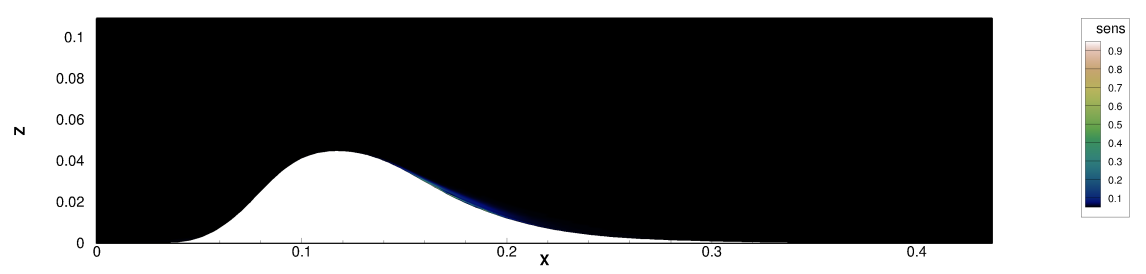

Structural sensitivity map.

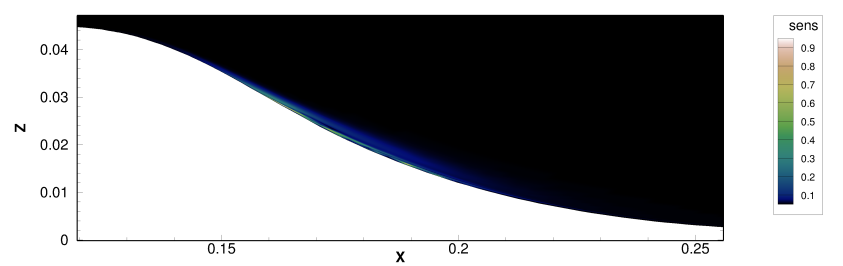

Structural sensitivity map, zoomed in.

Figure 6.12: The structural sensitivity map of the most unstable mode, 2-parameter, objective: pressure loss. 
velocity in the domain. We can see that the detachment is totally eleminated. In addition, the pressure loss $P_{\text {loss }}=0.663 \%$, which is larger than the pressure loss in the optimal configuration in the former optimisation investigation where the objective is minimising the pressure loss. We can see that, when considering the amplification rate as the objective of the optimisation, the optimisation provides a more stable mode, while it does not work very well on minimising the pressure loss, compared with the optimisation aiming at minimising the pressure loss.

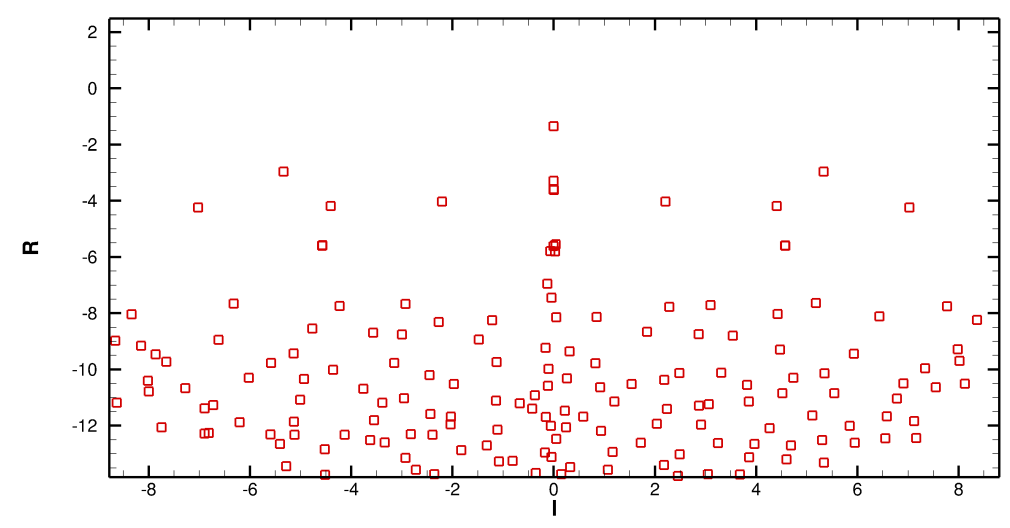

Figure 6.13: The eigenvalue spectrum of the optimal configuration, 2-parameter, objective: amplification rate.

\subsubsection{Results: 3-parameter optimisation}

In this section, we introduce a thirt parameter, $\alpha_{b}$, the angle of injection to the set of optimisation parameters. The geometry near the injection is shown in figure 6.17. Thus, the injection is charaterised by three parameter, $x_{b}, R_{b}$ and $\alpha_{b}$. The optimisation problem is modelled as in equation 6.1.

As for the investigations in this section, $P_{\text {loss }}(p)$ is still the pressure loss, and $\sigma_{R}(p)$ is still the growth rate (real part of the eigenvalue) both governed by the geometry parameter array $\boldsymbol{p}=\left(x_{b}, R_{b}, \alpha_{b}\right)$, instead of $\boldsymbol{p}=\left(x_{b}, R_{b}\right)$. Considering that a new optimisation parameter is introduced into consideration, the computational cost grows expotentially. So, we adopt an orthogonal design of experiments, with a dynamically update scheme to the surrogate model, to reduce the computational cost and to ensure the accuracy of the surrogate model. Some modifications are made to the design space as well, detailed in table 6.5.

\subsubsection{Optimisation aiming on reducing the pressure loss}

As in section 6.3.1.1, in this section, the objective of the optimisation is minimising the pressure loss, except for an additional parameter $\alpha_{b}$. The optimal injection 

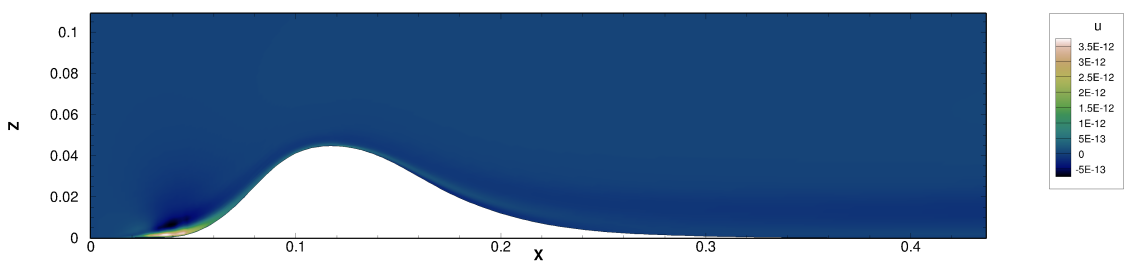

$u$.
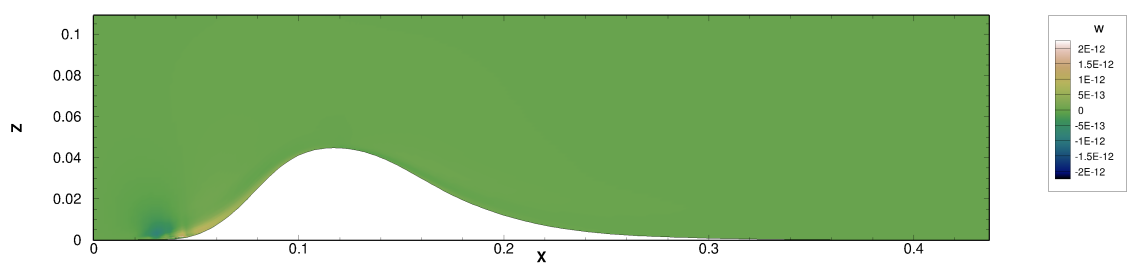

$w$.
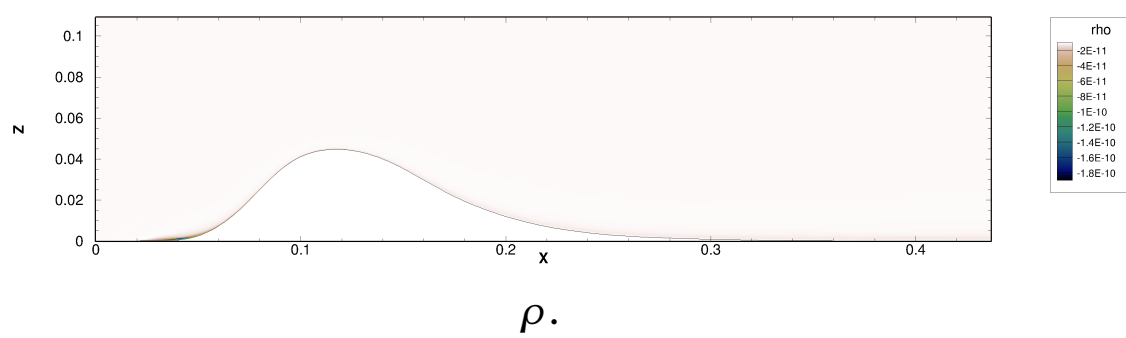

Figure 6.14: The most unstable eigenmode of the optimal design, 2-parameter, objective: amplification rate.

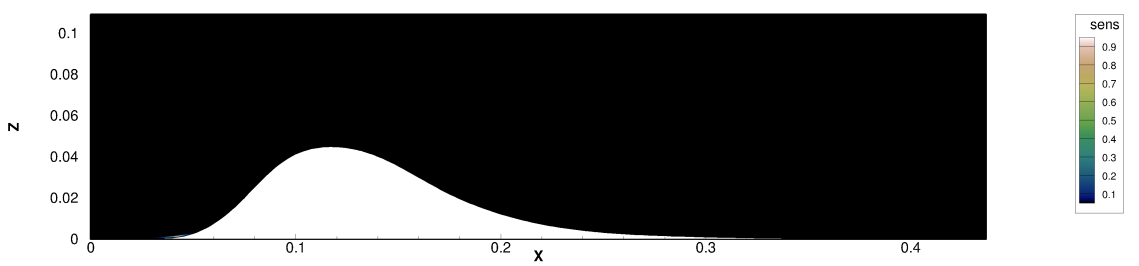

Structural sensitivity map.

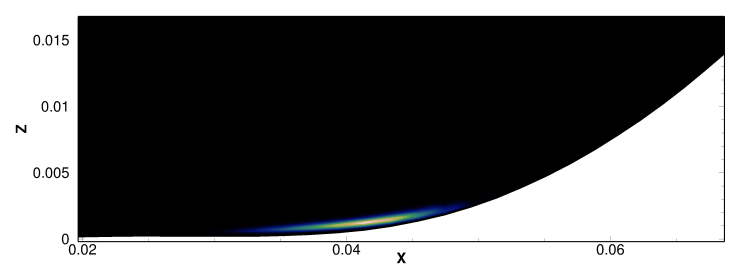

Structural sensitivity map, zoomed in.

Figure 6.15: The structural sensitivity map of the most unstable mode, 2-parameter, objective: amplification rate. 

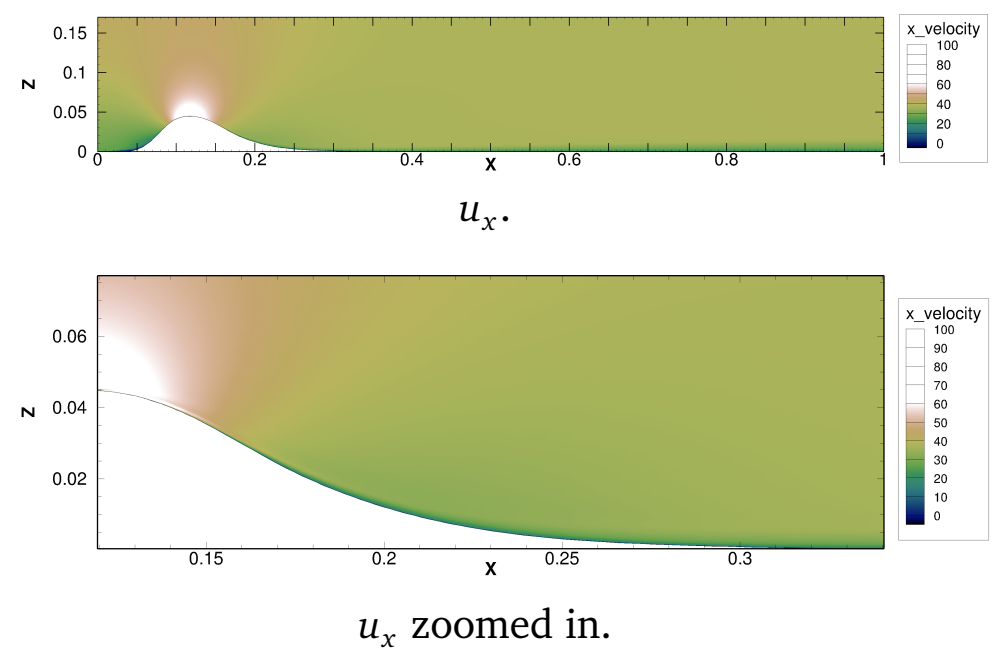

Figure 6.16: $u_{x}$, optimal design, 2-parameter, objective: amplification rate.

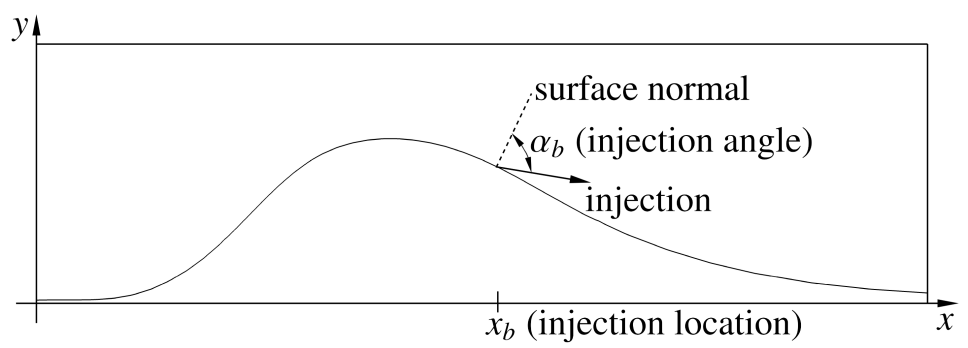

Figure 6.17: Geometric configuration used in the optimisation (zoomed in near the hump), 3-parameter.

Table 6.5: Design of experiment.

\begin{tabular}{c|c|c|c} 
Parameter & Number of levels & Minimum & Maximum \\
\hline$x_{b}$ & 7 & 0.1 & 0.6 \\
$R_{b}$ & 9 & 0 & 20 \\
$\alpha_{b}$ & 9 & $-80^{\circ}$ & $80^{\circ}$
\end{tabular}


configuration lies on the point where $x_{b}=0.121, R_{b}=5.94 \%$ and $\alpha_{b}=80^{\circ}$, which reduces the pressure loss to $P_{\text {loss }}=0.233 \%$. Comparing to that in section 6.3.1.1, the pressure loss is even smaller, which means a smaller angle of injection to the surface wall along the streamwise direction, reduces the pressure loss along the passage. As shown in figure 6.18, there is no detachment along the passage on the aft portion of the hump. Hence, by introducing an injection angle, the detachment can be eleminated and there is greater potential in reducing the pressure loss with the optimisation.
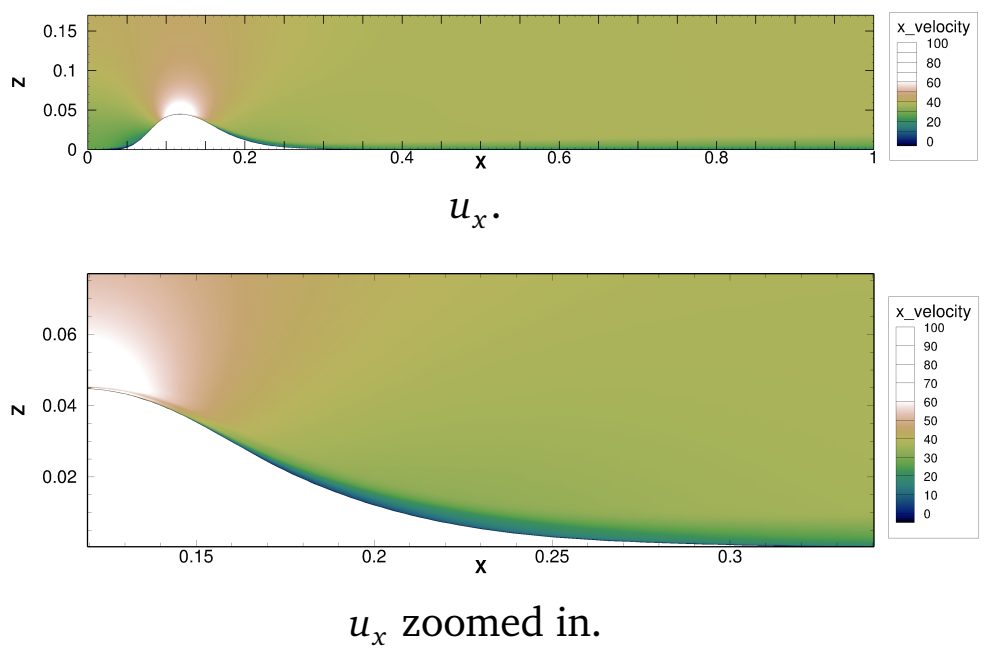

Figure 6.18: $u_{x}$, optimal design, 3-parameter, objective: pressure loss.

As usual, stability analysis and sensitivity analysis are carried out on the optimal configuration to verify the result. As shown in the eigenspecturm in figure 6.19 , all the modes have amplification rates below zero. By optimisation with the pressure loss as the objective, the modes are kept stable. As shown in figure 6.20, the most unstable mode is also relocated to the front of the hump, as well as the most sensitive region of this mode, as shown in figure 6.21 .

\subsubsection{Optimisation aiming on delaying instability}

In this part, we conduct an optimisation investigation with three parameters $x_{b}$, $R_{b}$ and $\alpha_{b}$, with minimising the amplification rate of the most unstable mode that causes the detachment. The optimisation is modelled as in equaiton 6.2. The optimisation investigation discussed here in this section is not different that in section 6.3.2.1, expect for the objective is changed to minimising the amplification rate of the most unstable mode.

First, as shown in figure 6.22 is the eigenspectrum of the optimal configuration. We can see that no eigenvalue has a positive real part, i.e. all modes have negative amplification rate. Compared with the amplification rate of the most unstable mode in section 6.3.2.1, the amplification rate here with minimising the amplification 


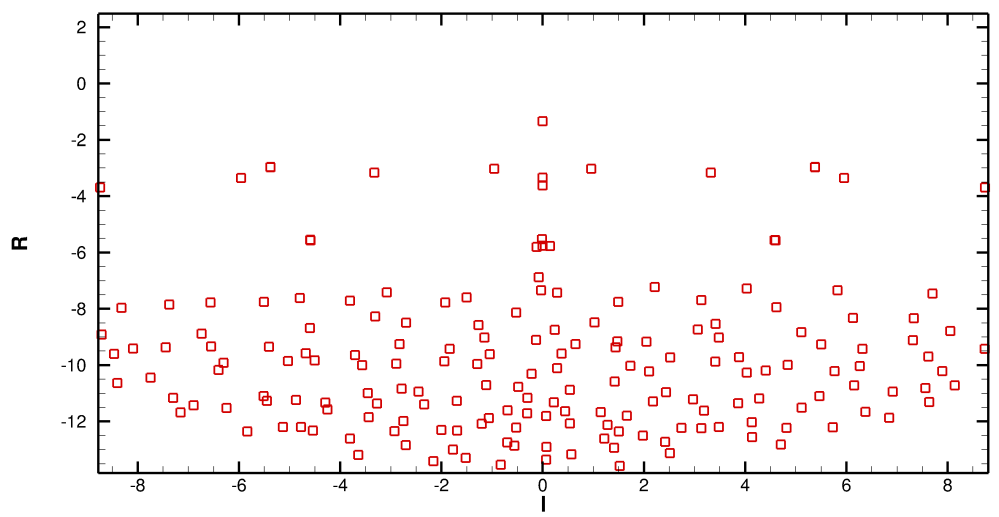

Figure 6.19: The eigenvalue spectrum of the optimal configuration, 3-parameter, objective: pressure loss.
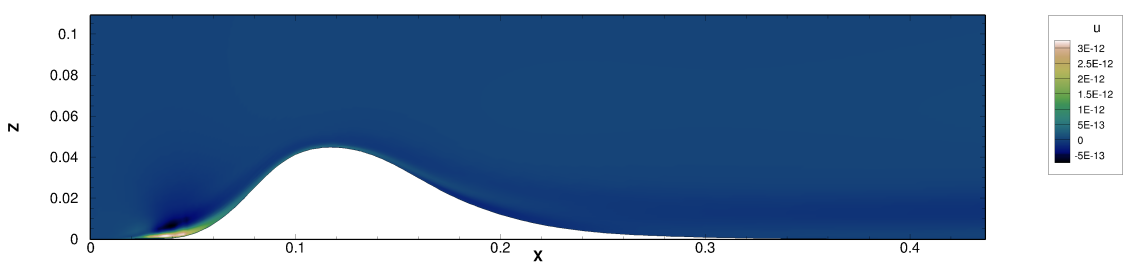

$u$.
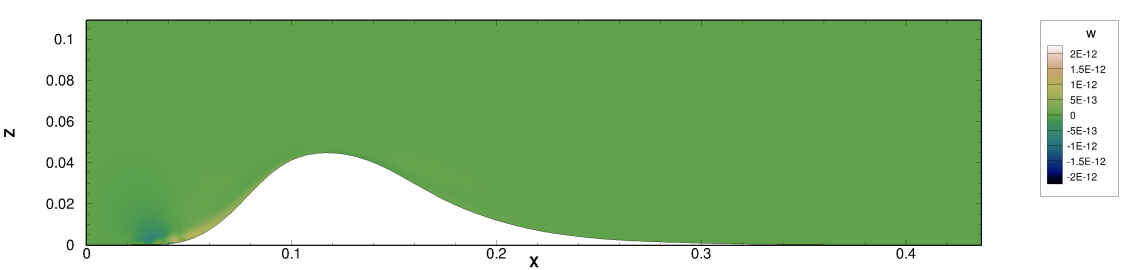

$w$.
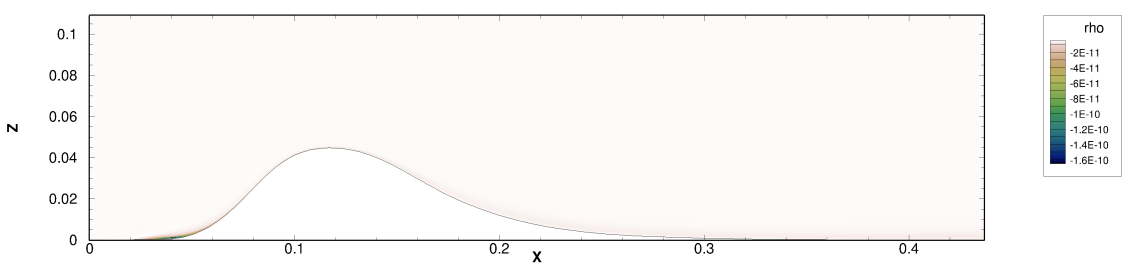

$\rho$.

Figure 6.20: The most unstable eigenmode of the optimal design, 3-parameter, objective: pressure loss. 


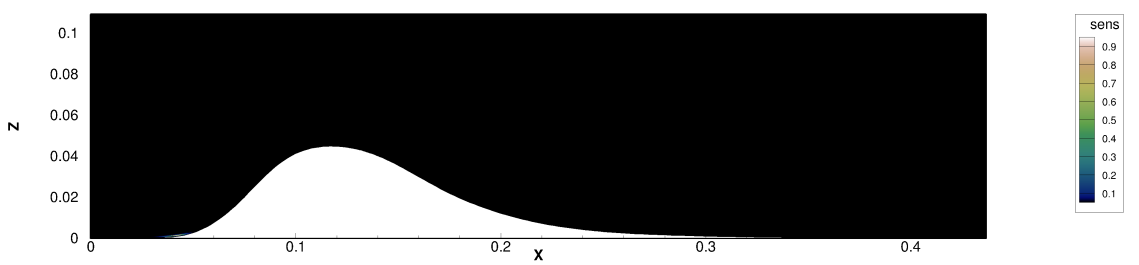

Structural sensitivity map.
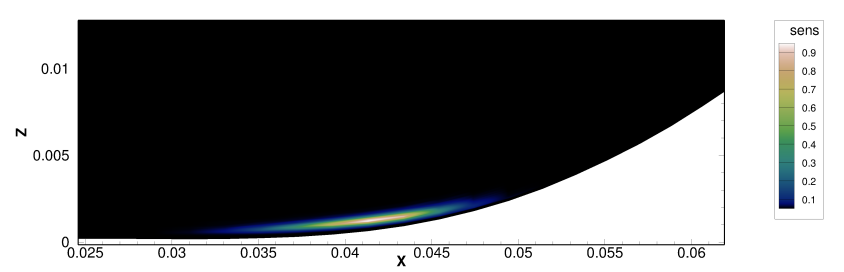

Structural sensitivity map, zoomed in.

Figure 6.21: The structural sensitivity map of the most unstable mode, 3-parameter, objective: pressure loss.

rate as the objective in this section, is slightly, yet smaller, which is the same case as discussed in section 6.3.1. The most unstable mode is shown in figure 6.23, from which we can see that the mode is also relocated to the front of the hump, as well as the most sensitive region of this mode, shown in figure 6.24.

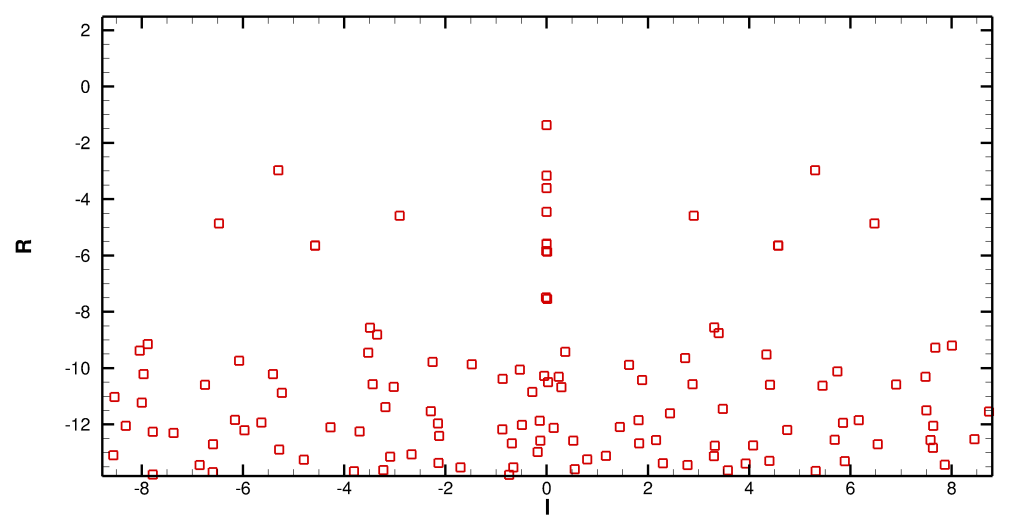

Figure 6.22: The eigenvalue spectrum of the optimal configuration, 3-parameter, objective: amplification rate.

Shown in figure 6.23, is the most unstable mode that could cause detachment. We can see that, In figure 6.24 is the structural sensitivity map, we can see that the most sensitive region of the most unstable mode is shifted to the front of the hump.

In figure 6.25 , we show $u_{x}$ of the flow field. We can see that, via optimisation with minimising the amplification rate of the most unstable mode that could cause 

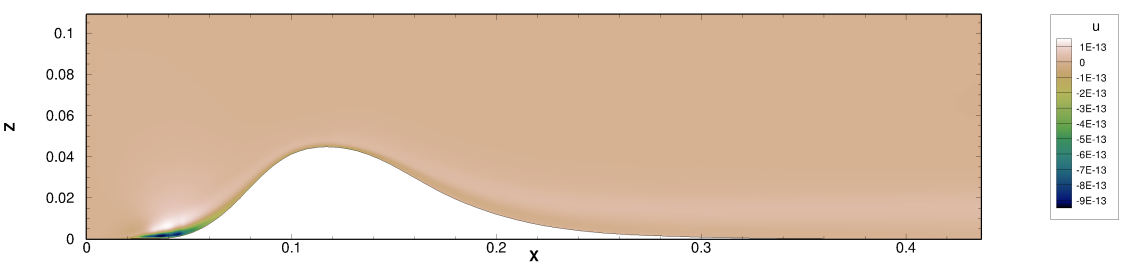

$u$.
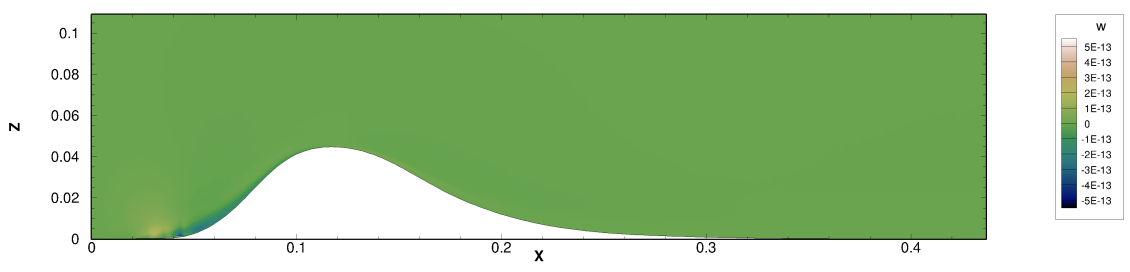

$w$.
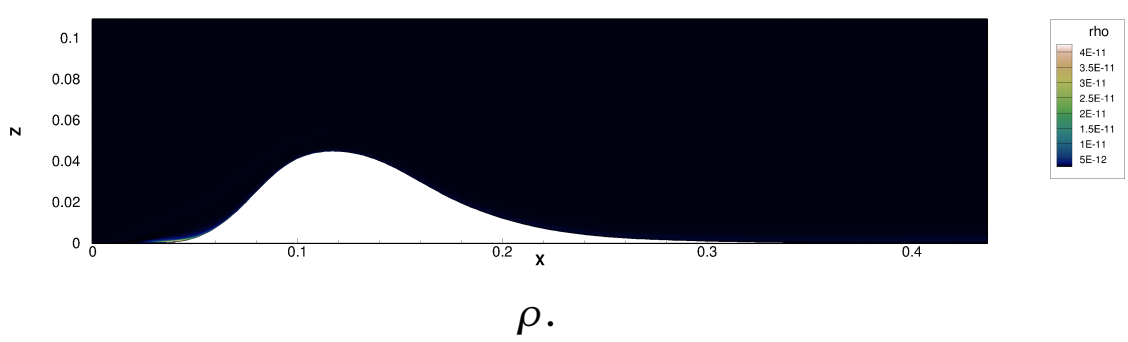

Figure 6.23: The most unstable eigenmode of the optimal design, 3-parameter, objective: amplification rate.

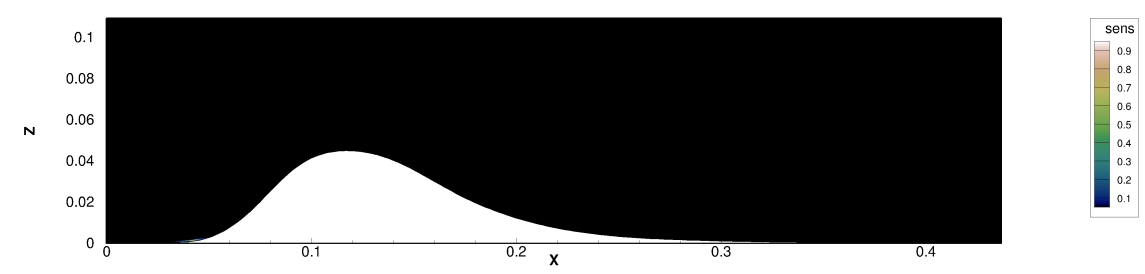

Structural sensitivity map.

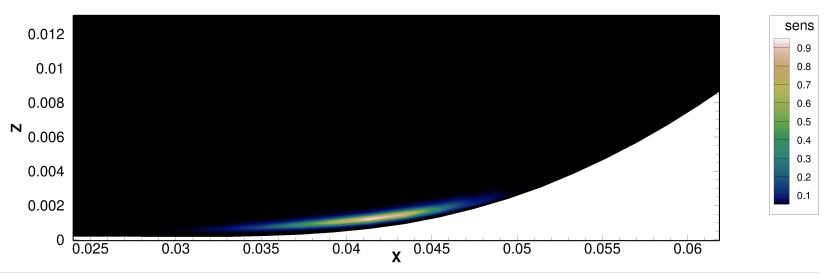

Structural sensitivity map, zoomed in.

Figure 6.24: The structural sensitivity map of the most unstable mode, 3-parameter, objective: amplification rate. 
detachment, the detachment in the domain can be eleminated. However, as is the case in section 6.3.1, the pressure loss here, $P_{\text {loss }}=0.591 \%$, is larger than that in the optimisation with minimising the pressure loss as the objective, discussed in section 6.3.2.1, where $P_{\text {loss }}=0.233 \%$
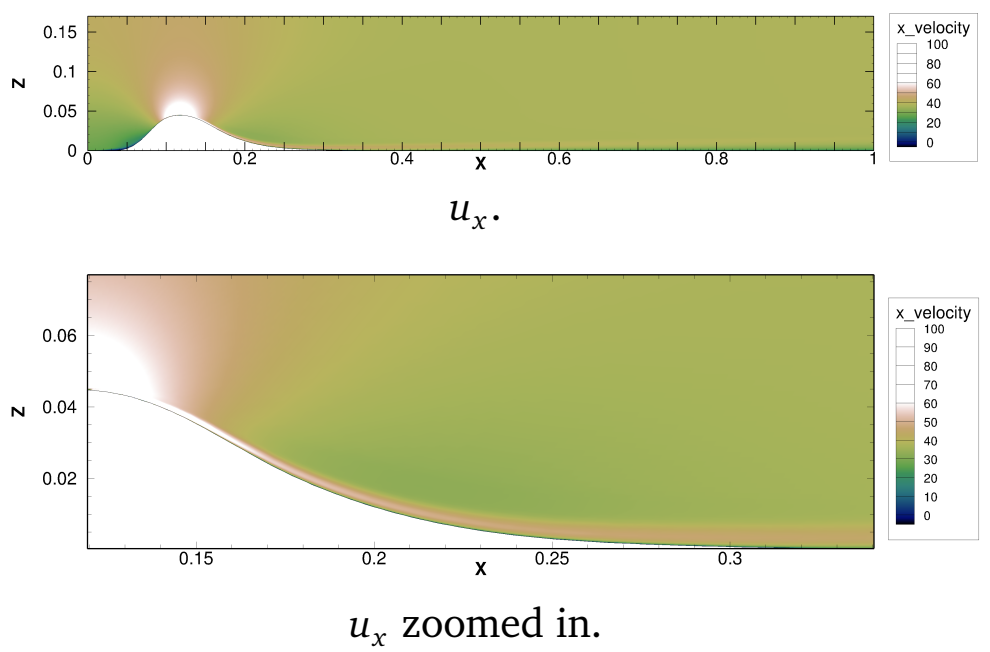

Figure 6.25: $u_{x}$, optimal design, 3-parameter, objective: amplification rate.

\subsection{Conclusions}

- The boundary layer detachment phenomenon in an expansion diffusion process as the one present in the aft portion of the suction side in a low pressure turbine is analysed.

- The eigenmodes associated to the boundary layer detachment are identified through BiGlobal stability analysis together with the modes that have deeper influence on the growth and distribution of the recirculated flow region.

- Through different optimisation investigations, optimal configurations of injection under different circumstances are obtained.

- Some of the optimal configurations successfully relocate the most unstable mode and its most sensitive region in the flow field.

- Summary of the different optimisation investigations:

In table 6.6 , case 1 is the two parameter optimisation with minimising the pressure loss as the objective, as described in section 6.3.1.1; case 2 is the two parameter optimisation with minimising the amplification rate of the most unstable mode as the objective, as decribed in section 6.3.1.2; case 3 is the three parameter optimisation with minimising the pressure loss as the 


\begin{tabular}{r|c|c|c|c} 
Behaviour & Case 1 & Case 2 & Case 3 & Case 4 \\
\hline$P_{\text {loss }}$ & $0.375 \%$ & $0.663 \%$ & $0.233 \%$ & $0.591 \%$ \\
$\sigma_{R}$ & -0.9753 & -1.347 & -1.343 & -1.372 \\
Mode, sensitivity relocate & No & Yes & Yes & Yes
\end{tabular}

Table 6.6: Summary of the different optimisation investigations.

objective, as described in section 6.3.2.1; case 4 is the three parameter optimisation with minimising the amplification rate of the most unstable mode as the objective, as decribed in section 6.3.2.2. 


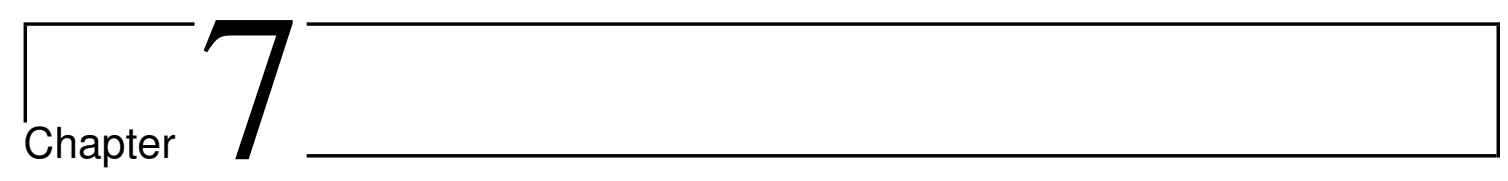

\section{Conclusions and Outlook}

\section{Contents}

7.1 Conclusions and Summary . . . . . . . . . . 99

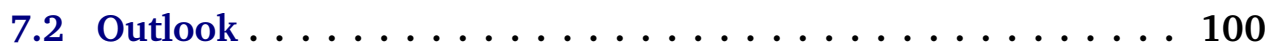

\subsection{Conclusions and Summary}

An efficient aerodynamic optimisation tool based on stability analysis and sensitivity analysis is successfully applied to stabilities problems, and offering optimal flow control strategies in both passive and active flow control. To make this optimisation approach feasible, geometric parametrisation is used to represent the geometric with a limited number of parameters; a mesh deformation tool based free form deformation (FFD) is developed, avoiding generating large numbers of similar meshes from scratch; a dynamically updated surrogate model is developed to ensure the accuracy of the surrogate model, while reducing the sampling points in the design of experiments; a mode tracking scheme is developed to avoid manual checking and idenfying modes of interest, which reduces computational cost and alleviates manual labour.

In the contraction channels, the bifurcation is confirmed, which is found to be controlled by asymmetric global modes, by conducting stability analysis. Structural sensitivity analyses shows the regions in which these modes are most affected. By paramtrising the boundary of the contraction channel, optimisation investigations are conducted, which give the optimal set of parameters of the geometry, giving optimal designs that endure much larger Reynolds numbers without the onset of instability. Through the shape deformation provided by the optimisation, the most sensitivity region of the mode that causes the bifurcation to the flow is shifted to another location.

In the wall-mounted hump problem, the intresting fact that with lower Reynolds number, the detachment grows is confirmed. Stability analysis and sensitivity analysis provides the mode that is causing the detachment and the region in which this 
mode is most easily manipulated. Optimisation investigations gives the optimal injection configurations that both supress the mode that is causing the instability and also minimise the pressure loss in the passage. Like that in the investigations in the contraction channels, the most sensitive region of the most unstable mode is also shifted from the original location.

The results of the optimisation investigations show that the optimisation tool that is based on stability analysis and sensitivity analysis is capable of providing an optimal passive/active flow control strategy. Additionally, the computational cost is controlled in a reasonable range with the assistance of a dynamically updated surrogate model. The mode tracking scheme based on MAC (Modal Assurance Criterion) effectively recognises the modes that is of interest in the investigations.

\subsection{Outlook}

There is much more potential in design optimisation and stability analysis and sensitivity analysis that can be taken into consideration when seeking an optimal flow control method.

Multi-objective optimisation methods can be adopted in the aerodynamic optimisation based on stability analysis and sensitivity analysis. Thus, more than one objectives can be taken into account, and theoritically, find even better solutions.

In this thesis, due to the complexity of the problems, the optimisation investigations only adopt gradient-free methods. In the future, a gradient-based method could be utilised and better support the multi-objective optimisation investigations.

Currently, a buffet phenomenon around the NACA0012 airfoil is being studied as a multi-objective optimisation problem. One objective is to minimise the amplification rate of the mode that is causing the buffet phenomenon, the other is to minimise the drag (or drag increase, which is caused by the hump or dimple that is introduced to suppress the mode that is causing the buffet phenomenon). The adjoint approach is adopted to get the gradient information of the design parameters to the two cost funtions.

More flow control problems will be found suitable to be solved by the optimisation methodology introduced in this thesis, and more techniques could be developed to better accomplish this task. 


\section{Bibliography}

[1] S. Albensoeder, H. C. Kuhlmann, and H. J. Rath. Three-dimensional centrifugal-flow instabilities in the lid-driven-cavity problem. Physics of Fluids, 13(1):121-135, 2001. Cited on page(s) 3, 47.

[2] F. Alizard and J.-C. Robinet. Spatially convective global modes in a boundary layer. Physics of fluids, 19(11):114105, 2007. Cited on page(s) 23.

[3] S. R. Allmaras and F. T. Johnson. Modifications and clarifications for the implementation of the spalart-allmaras turbulence model. In Seventh international conference on computational fluid dynamics (ICCFD7), pages 1-11, 2012. Cited on page(s) 9, 11.

[4] K. Amadori, C. Jouannet, and P. Krus. A Framework for Aerodynamic and Structural Optimization in Conceptual Design. Cited on page(s) 1.

[5] B. Aupoix and P. Spalart. Extensions of the spal. art-allmaras turbulence model to account for wall. Engineering Turbulence Modelling and Experiments 5, page 187, 2002. Cited on page(s) 10.

[6] D. Barkley, M. G. M. Gomes, and R. D. Henderson. Three-dimensional instability in flow over a backward-facing step. Journal of Fluid Mechanics, (473):167-190, 2002. Cited on page(s) 3, 47.

[7] J. P. Bons, R. Sondergaard, and R. B. Rivir. The Fluid Dynamics of LPT Blade Separation Control Using Pulsed Jets. 124(January 2002):77-85, 2016. Cited on page(s) 76 .

[8] A. Borzì and V. Schulz. Multigrid methods for pde optimization. SIAM review, 51(2):361-395, 2009. Cited on page(s) 20.

[9] L. Brandt. The lift-up effect: The linear mechanism behind transition and turbulence in shear flows. European Journal of Mechanics - B/Fluids, 47:80 - 96, 2014. Enok Palm Memorial Volume. Cited on page(s) 57. 
[10] O. M. F. Browne, G. Rubio, E. Ferrer, and E. Valero. Sensitivity analysis to unsteady perturbations of complex flows: A discrete approach. International Journal for Numerical Methods in Fluids, 76(12):1088-1110, 2014. Cited on page(s) $3,23,28,31,33,77$.

[11] A. R. Byerley, O. Sto“ rmer, J. W. Baughn, T. W. Simon, K. W. Van Treuren, and J. r. List. Using gurney flaps to control laminar separation on linear cascade blades. J. Turbomach., 125(1):114-120, 2003. Cited on page(s) 76.

[12] K. K. Chen, C. W. Rowley, and H. A. Stone. Vortex dynamics in a pipe Tjunction: Recirculation and sensitivity. Physics of Fluids, 2015. Cited on page(s) 3, 47.

[13] W. Cherdron, F. Durst, and J. H. Whitelaw. Asymmetric flows and instabilities in symmetric ducts with sudden expansions. Journal of Fluid Mechanics, 84(01):13, 1978. Cited on page(s) 46, 47.

[14] T. P. Chiang, A. Sau, and R. R. Hwang. Asymmetry and bifurcations in three-dimensional sudden-contraction channel flows. Physical Review E Statistical, Nonlinear, and Soft Matter Physics, 2011. Cited on page(s) 2, 47, 70, 72 .

[15] T. P. Chiang and T. W. H. Sheu. Bifurcations of Flow Through Plane Symmetric Channel. Journal of Fluids Engineering, 124(June):444-451, 2002. Cited on page(s) 2, 46, 47, 48, 49, 50, 52.

[16] J.-M. Chomaz. Global Instabilities in Spatially Developing Flows: NonNormality and Nonliearity. Annual Review of Fluid Mechanics, 37(1):357392, 2005. Cited on page(s) 32, 33.

[17] L. Davis, editor. Handbook of Genetic Algorithms. Van Nostrand Reinhold, 1991. Cited on page(s) 36.

[18] J. de Vicente Buendía and E. Valero. Spectral multi-domain method for the global instability analysis of complex cavity flows. ETSIAE UPM Madrid, 2010. Cited on page(s) 23.

[19] S. Dennis and F. Smith. Steady flow through a channel with a symmetrical constriction in the form of a step. Proc. R. Soc. Lond. A, 372:393-414, 1980. Cited on page(s) $46,48,49$.

[20] P. G. Drazin and W. H. Reid. Hydrodynamic Stability. Cambridge Mathematical Library. Cambridge University Press, 2 edition, 2004. Cited on page(s) 46. 
[21] F. Durst, W. F. Schierholz, and A. M. Wunderlich. Experimental and Numerical Investigations of Plane Duct Flows With Sudden Contraction. Journal of Fluids Engineering, 109(4):376-373, 1987. Cited on page(s) 46.

[22] W. Edwards, L. S. Tuckerman, R. A. Friesner, and D. Sorensen. Krylov methods for the incompressible navier-stokes equations. Journal of computational physics, 110(1):82-102, 1994. Cited on page(s) 31.

[23] A. Fani, S. Camarri, and M. Salvetti. Stability analysis and control of the flow in a symmetric channel with a sudden expansion. Physics of Fluids, 24, 08 2012. Cited on page(s) 2, 47.

[24] R. M. Fearn, T. Mullin, and A. K. Cliffe. Nonlinear flow phenomena in a symmetric sudden expansion. Journal of Fluid Mechanics, 211:595-608, 1990. Cited on page(s) 2, 47.

[25] E. Ferrer, O. M. Browne, and E. Valero. Sensitivity analysis to control the far-wake unsteadiness behind turbines. Energies, 10, 2017. Cited on page(s) $3,33,77$.

[26] E. Ferrer, J. de Vicente, and E. Valero. Low cost 3D global instability analysis and flow sensitivity based on dynamic mode decomposition and highorder numerical tools. International Journal for Numerical Methods in Fluids, 76(3):169-184, sep 2014. Cited on page(s) 3, 31, 77.

[27] T. L. Flinois and T. Colonius. Optimal control of circular cylinder wakes using long control horizons. Physics of Fluids, 2015. Cited on page(s) 2.

[28] J. A. Franck and T. Colonius. Compressible large-eddy simulation of separation control on a wall-mounted hump. AIAA journal, 48(6):1098-1107, 2010. Cited on page(s) 78.

[29] F. Gallaire, M. Marquillie, and U. Ehrenstein. Three-dimensional transverse instabilities in detached boundary layers. Journal of Fluid Mechanics, 571:221-233, 2007. Cited on page(s) 3, 47.

[30] P. Garabedian and G. McFadden. Design of supercritical swept wings. AIAA Journal, 20(3):289-291, 1982. Cited on page(s) 2.

[31] F. M. Garrido, E. V. Sánchez, and J. de Vicente Buendıa. Instability analysis of incompressible open cavity flows. ETSIAE UPM Madrid, 2014. Cited on page(s) 23.

[32] F. Gianetti and P. Luchini. Structural sensitivity of the first instability of the cylinder wake. Journal of Fluid Mechanics, 581:167-197, 2007. Cited on page(s) $3,32,33,77$. 
[33] F. Giannetti and P. Luchini. Structural sensitivity of the first instability of the cylinder wake. Journal of Fluid Mechanics, 581(1):167-197, 2007. Cited on page(s) 23, 31, 33.

[34] C. Goldberg, D. Nalianda, D. MacManus, and P. Pilidis. Installed Performance Assessment of a Boundary Layer Ingesting Distributed Propulsion System at Design Point. pages 1-22, 2016. Cited on page(s) 76.

[35] L. Gonzalez, E. Ferrer, and H. Diaz-Ojeda. Onset of three-dimensional flow instabilities in lid-driven circular cavities. Physics of Fluids, 29(6):064102, 2017. Cited on page(s) 3, 77.

[36] D. Greenblatt and I. J. Wygnanski. Control of flow separation by periodic excitation, 2000. Cited on page(s) 76.

[37] Y. Guevel, T. Allain, G. Girault, and J. M. Cadou. Numerical bifurcation analysis for 3-dimensional sudden expansion fluid dynamic problem. International Journal for Numerical Methods in Fluids, 2018. Cited on page(s) 2, 47, 70, 72 .

[38] Z.-H. Han and K.-S. Zhang. Surrogate-Based Optimization. Real-World Applications of Genetic Algorithms, pages 343-362, 2008. Cited on page(s) 37.

[39] S. Haque, I. Lashgari, F. Giannetti, and L. Brandt. Stability of fluids with shear-dependent viscosity in the lid-driven cavity. Journal of Non-Newtonian Fluid Mechanics, 173-174:49-61, 2012. Cited on page(s) 3, 47.

[40] T. Hawa and Z. Rusak. The dynamics of a laminar flow in a symmetric channel with a sudden expansion. Journal of Fluid Mechanics, 436:283-320, 2001. Cited on page(s) 52.

[41] D. M. Hawken, P. Townsend, and M. F. Webster. Numerical simulation of viscous flows in channels with a step. Computers and Fluids, 20(1):59-75, 1991. Cited on page(s) 46, 48, 49.

[42] P. A. Henne. Inverse transonic wing design method. Journal of Aircraft, 18(2):121-127, 1981. Cited on page(s) 1.

[43] T. Herbert. Parabolized stability equations. Annual Review of Fluid Mechanics, 29(1):245-283, 1997. Cited on page(s) 25.

[44] D. Hill. Adjoint systems and their role in the receptivity problem for boundary layers. Journal of Fluid Mechanics, 292:183-204, 1995. Cited on page(s) 31.

[45] D. C. Hill. A theoretical approach for analyzing the restabilization of wakes. In AIAA Aersoapce Science Meeting Exhib. 30th, Reno, NV, AIAA Pap., volume 0067, 1992. Cited on page(s) 3, 31, 77. 
[46] H. Hodson and R. Dominy. The off-design performance of a low-pressure turbine cascade. 1987. Cited on page(s) 78.

[47] J. H. Holland. Adaptation in Nature and Artificial Systems. University of Michigan Press, 1975. Cited on page(s) 36.

[48] J. Huang, T. C. Corke, and F. O. Thomas. Unsteady plasma actuators for separation control of low-pressure turbine blades. AIAA Journal, 44(7):14771487, 2006. Cited on page(s) 76.

[49] L. Hultgren and D. Ashpis. Demonstration of Separation Delay with Glow Discharge Plasma Actuators. 2013. Cited on page(s) 76.

[50] R. Hunt. The numerical solution of the laminar flow in a constricted channel at moderately high Reynolds number using Newton iteration. International Journal for Numerical Methods in Fluids, 11(2):247-259, 1990. Cited on page(s) 46, 48, 49.

[51] D. Y. Hur, M.-h. Kwak, S.-H. Yun, and D. Lee. Aerodynamic optimization on the conceptual design stage. 01 2011. Cited on page(s) 1.

[52] L. Huyse, S. L. Padula, R. M. Lewis, and W. Li. Probabilistic approach to free-form airfoil shape optimization under uncertainty. AIAA Journal, 40(9):1764-1772, 2002. Cited on page(s) 2, 77.

[53] M. Ilak, P. Schlatter, S. Bagheri, and D. Henningson. Bifurcation and stability analysis of a jet in crossflow. part 1: Onset of global instability at a low velocity ratio. J. Fluid Mech, 696:94-121, 2011. Cited on page(s) 25.

[54] M. Iorio, L. González, and E. Ferrer. Direct and adjoint global stability analysis of turbulent transonic flows over a naca0012 profile. International Journal for Numerical Methods in Fluids, 76(3):147-168, 2014. Cited on page(s) 23.

[55] M. C. Iorio. Global stability analysis of turbulent transonic flows on airfoil geometries. PhD thesis, Universidad Politécnica de Madrid, 2015. Cited on page(s) 23, 33.

[56] M. C. Iorio, L. M. González, and E. Ferrer. Direct and adjoint global stability analysis of turbulent transonic flows over a NACA0012 profile. International Journal for Numerical Methods in Fluids, 76(3):147-168, 2014. Cited on page(s) 3, 77.

[57] P. S. Iyer and M. R. Malik. Wall-modeled large eddy simulation of flow over a wall-mounted hump. In 46th AIAA Fluid Dynamics Conference, page 3186, 2016. Cited on page(s) 78. 
[58] A. Jameson. Advances in aerodynamic shape optimization. In C. Groth and D. W. Zingg, editors, Computational Fluid Dynamics 2004, pages 687-698, Berlin, Heidelberg, 2006. Springer Berlin Heidelberg. Cited on page(s) 1.

[59] A.-H. Jiang, X.-C. Huang, Z.-H. Zhang, J. Li, Z.-Y. Zhang, and H.-X. Hua. Uncertainty quantification of squeal instability via surrogate modelling. Mechanical Systems and Signal Processing, 24(8):2947-2960, 2010. Cited on page(s) 41.

[60] M. Kumar, M. Husian, N. Upreti, and D. Gupta. Genetic Algorithm: Review and Application. International Journal of Information Technology and Knowledge Management, 2(2):451-454, 2010. Cited on page(s) 36.

[61] B. Laborie, F. Rouyer, D. E. Angelescu, and E. Lorenceau. On the stability of the production of bubbles in yield-stress fluid using flow-focusing and Tjunction devices. Physics of Fluids, 2016. Cited on page(s) 3, 47.

[62] J. Lake, P. King, and R. Rivir. Reduction of Separation Losses on a Turbine Blade with Low Reynolds Numbers. 37th Aerospace Sciences Meeting and Exhibit, page 242, 1999. Cited on page(s) 76.

[63] D. Lanzerstorfer and H. C. Kuhlmann. Global stability of the twodimensional flow over a backward-facing step. Journal of Fluid Mechanics, 693(November 2011):1-27, 2011. Cited on page(s) 3, 47.

[64] I. Lashgari, O. Tammisola, V. Citro, M. P. Juniper, and L. Brandt. The planar X-junction flow: stability analysis and control. Journal of Fluid Mechanics, 753(August):1-28, 2014. Cited on page(s) 3, 47, 50, 52, 56, 61.

[65] M. J. Lighthill. A new method of two-dimensional aerodynamics design. In RM1111, Aeronautical Research Council, 1945. Cited on page(s) 1.

[66] P. Luchini and A. Bottaro. Adjoint Equations in Stability Analysis. Annual Review of Fluid Mechanics, 46(1):493-517, 2014. Cited on page(s) 3, 33, 77.

[67] T. J. Mackman and C. B. Allen. Comparison of Parameter Tuning Strategies for RBF Surrogate Models with Adaptive Sampling. Evaluation, (June):114, 2011. Cited on page(s) 37.

[68] O. Marquet and L. Lesshafft. Identification of contributing flow regions for linear and nonlinear flow instabilities. arXiv preprint, 2015. Cited on page(s) 33.

[69] O. Marquet, D. Sipp, and L. Jacquin. Sensitivity analysis and passive control of cylinder flow. Journal of Fluid Mechanics, 615:221-252, 2008. Cited on page(s) $3,47,77$. 
[70] A. Martinez-Cava, M. Chavez-Modena, E. Valero, J. De Vicente, and E. Ferrer. Sensitivity gradients of surface geometry modifications based on stability analysis of compressible flows. Physical Review Fluids, 5, 2020. Cited on page(s) 2 .

[71] F. Menter. Zonal two equation kw turbulence models for aerodynamic flows. In 23rd fluid dynamics, plasmadynamics, and lasers conference, page 2906, 1993. Cited on page(s) 11.

[72] C. Mettot, F. Renac, and D. Sipp. Computation of eigenvalue sensitivity to base flow modifications in a discrete framework: Application to open-loop control. Journal of Computational Physics, 269:234 - 258, 2014. Cited on page(s) 33.

[73] J. Mizushima and Y. Shiotani. Transitions and instabilities of flow in a symmetric channel with a suddenly expanded and contracted part. Journal of Fluid Mechanics, 434:355-369, 2001. Cited on page(s) 3, 47.

[74] B. R. Noack and H. Eckelmann. A global stability analysis of the steady and periodic cylinder wake. Journal of Fluid Mechanics, 270:297-330, 1994. Cited on page(s) 23.

[75] S. Obayashi and S. Takanashi. Genetic optimization of target pressure distributions for inverse design methods. AIAA Journal, 34(5):881-886, 1996. Cited on page(s) 2 .

[76] S. A. Orszag. Accurate solution of the orr-sommerfeld stability equation. Journal of Fluid Mechanics, 50(4):689-703, 1971. Cited on page(s) 24.

[77] I. Padilla, J. Saavedra, G. Paniagua, and F. Pinna. Analysis of the Boundary Layer Stability to Assess Flow Separation Control Capability in Low-Pressure Turbines. 6th European Conference on Computational Fluid Dynamics, 2018. Cited on page(s) 76 .

[78] P. Y. Passaggia, T. Leweke, and U. Ehrenstein. Transverse instability and low-frequency flapping in incompressible separated boundary layer flows: An experimental study. Journal of Fluid Mechanics, 703:363-373, 2012. Cited on page(s) 3, 47.

[79] J. Périaux. Optimization Methods and Tools for Multi Objectives DESIGN using Evolutionary Algorithms and Game strategies. pages 1-44, 2014. Cited on page(s) 2, 77 .

[80] J. Poggie. DC Glow Discharges: A Computational Study for Flow Control Applications. 2012. Cited on page(s) 76. 
[81] D. Postl and H. F. Fasel. Direct numerical simulation of turbulent flow separation from a wall-mounted hump. AIAA journal, 44(2):263-272, 2006. Cited on page(s) 78.

[82] J. O. Pralits, L. Brandt, and F. Giannetti. Instability and sensitivity of the flow around a rotating circular cylinder. Journal of Fluid Mechanics, 2010. Cited on page(s) 33.

[83] J. O. Pralits and A. Hanifi. Optimization of steady suction for disturbance control on infinite swept wings. Physics of Fluids, 2003. Cited on page(s) 2, 77.

[84] N. V. Queipo, R. T. Haftka, W. Shyy, T. Goel, R. Vaidyanathan, and P. Kevin Tucker. Surrogate-based analysis and optimization. Progress in Aerospace Sciences, 41(1):1-28, 2005. Cited on page(s) 37.

[85] N. Ramanan and G. M. Homsy. Linear stability of lid-driven cavity flow. Physics of Fluids, 6(8):2690-2701, 1994. Cited on page(s) 23.

[86] B. Raverdy, I. Mary, P. Sagaut, and N. Liamis. High-resolution largeeddy simulation of flow around low-pressure turbine blade. AIAA journal, 41(3):390-397, 2003. Cited on page(s) 78.

[87] D. Rodríguez and V. Theofilis. Structural changes of laminar separation bubbles induced by global linear instability. Journal of Fluid Mechanics, 655:280-305, 2010. Cited on page(s) 3, 47.

[88] Y. Saad. Numerical methods for large eigenvalue problems. Manchester University Press, 1992. Cited on page(s) 30.

[89] J. Saavedra and G. Paniagua. Transient Performance of Separated Flows: Characterization and Active Flow Control. Journal of Engineering for Gas Turbines and Power, 2019. Cited on page(s) 76.

[90] H. Salwen and C. E. Grosch. The continuous spectrum of the orr-sommerfeld equation. part 2. eigenfunction expansions. Journal of Fluid Mechanics, 104:445-465, 1981. Cited on page(s) 31.

[91] S. Sanvido. Stability analysis of complex aerodynamic flows. PhD thesis, Universidad Politécnica de Madrid, 2017. Cited on page(s) 20.

[92] H. Schlichting and K. Gersten. Boundary-layer theory. Springer, 2016. Cited on page(s) 24.

[93] P. J. Schmid. Nonmodal stability theory. Annu. Rev. Fluid Mech., 39:129-162, 2007. Cited on page(s) 25. 
[94] P. J. Schmid. Dynamic mode decomposition of numerical and experimental data. Journal of fluid mechanics, 656:5-28, 2010. Cited on page(s) 31.

[95] P. J. Schmid and D. S. Henningson. Stability and Transition in Shear Flows. Springer, 2001. Cited on page(s) 2, 46, 72, 77.

[96] P. J. Schmid, D. S. Henningson, and D. Jankowski. Stability and transition in shear flows. applied mathematical sciences, vol. 142. Appl. Mech. Rev., 55(3):B57-B59, 2002. Cited on page(s) 24, 25.

[97] A. Shahbani-Zahiri, H. Hassanzadeh, M. M. Shahmardan, and M. Norouzi. Investigation of pitchfork bifurcation phenomena effects on heat transfer of viscoelastic flow inside a symmetric sudden expansion. Physics of Fluids, 2017. Cited on page(s) 2, 47.

[98] D. Sipp, O. Marquet, P. Meliga, and A. Barbagallo. Dynamics and control of global instabilities in open flows: a linearized approach. Applied Mechanical reviews, 63(3):1-26, 2010. Cited on page(s) 3, 77.

[99] I. J. Sobey and P. G. Drazin. Bifurcations of two-dimensional channel flows. Journal of Fluid Mechanics, 171(6):263-287, 1986. Cited on page(s) 46, 47, 52 .

[100] H. SOBIECZKY, N. YU, K.-Y. FUNG, and A. SEEBASS. A new method for designing shock-free transonic configurations. Cited on page(s) 1.

[101] P. R. Spalart and S. R. Allmaras. One-equation turbulence model for aerodynamic flows. Recherche aerospatiale, 1994. Cited on page(s) 9.

[102] R. Stieger and H. Hodson. The transition mechanism of highly loaded lowpressure turbine blades. J. Turbomach., 126(4):536-543, 2004. Cited on page(s) 78.

[103] O. Tammisola. Optimal wavy surface to suppress vortex shedding using second-order sensitivity to shape changes. European Journal of Mechanics, B/Fluids, 2017. Cited on page(s) 63.

[104] V. Theofilis. Advances in global linear instability analysis of nonparallel and three-dimensional flows. Progress in aerospace sciences, 39(4):249-315, 2003. Cited on page(s) 23, 25, 31.

[105] V. Theofilis. Global linear instability. Annual Review of Fluid Mechanics, 43:319-352, 2011. Cited on page(s) 23.

[106] S. Thomas, T. Ameel, and J. Guilkey. Mixing kinematics of moderate Reynolds number flows in a T-channel. Physics of Fluids, 22(1):1-10, 2010. Cited on page(s) 3, 47. 
[107] T. TRANEN. A rapid computer aided transonic airfoil design method. Cited on page(s) 2 .

[108] L. S. Tuckerman and D. Barkley. Bifurcation analysis for timesteppers. In Numerical methods for bifurcation problems and large-scale dynamical systems, pages 453-466. Springer, 2000. Cited on page(s) 25, 31.

[109] R. J. Volino. Passive Flow Control on Low-Pressure Turbine Airfoils. Journal of Turbomachinery, 2003. Cited on page(s) 76.

[110] R. J. Volino and L. S. Hultgren. Measurements in separated and transitional boundary layers under low-pressure turbine airfoil conditions. J. Turbomach., 123(2):189-197, 2001. Cited on page(s) 78.

[111] J. Wang. Aerodynaimic shape optimization by the Stabkelberg game coupled with the continuous adjoint method. PhD thesis, Zhejiang University, 2018. Cited on page(s) 17, 77.

[112] Y. Wang, E. Ferrer, A. Martínez-Cava, Y. Zheng, and E. Valero. Enhanced stability of flows through contraction channels: Combining shape optimization and linear stability analysis. Physics of Fluids, 31(7):074109, 2019. Cited on page(s) 77.

[113] R. Wille and H. Fernholz. Report on the first European Mechanics Colloquium, on the Coanda effect. Journal of Fluid Mechanics, 23(4):801-819, 1965. Cited on page(s) 46, 52.

[114] Z. XIA, Z. ZHU, and L. WU. A computational method for inverse design of transonic airfoil and wing. Cited on page(s) 2 .

[115] D. Xiao and G. Papadakis. Nonlinear optimal control of bypass transition in a boundary layer flow. Physics of Fluids, 2017. Cited on page(s) 2.

[116] R. Yondo, E. Andrés, and E. Valero. A review on design of experiments and surrogate models in aircraft real-time and many-query aerodynamic analyses. Progress in Aerospace Sciences, 96:23 - 61, 2018. Cited on page(s) 39.

[117] V. N. Zhigulev and A. M. Tumin. Origin of turbulence: Dynamic theory of the excitation and evolution of instabilities in boundary layers. NoIzN, 1987. Cited on page(s) 31. 


